\title{
Charged Sectors, Spin and Statistics in Quantum Field Theory on Curved Spacetimes
}

\author{
D. Guido*ף, R. Longo**1, J.E. Roberts**1, R. Verch*** \\ * Dipartimento di Matematica, \\ Università della Basilicata, \\ I-85100 Potenza, Italy \\ e-mail: guido@unibas.it \\ ** Dipartimento di Matematica, \\ Università di Roma "Tor Vergata", \\ I-00133 Roma, Italy \\ e-mail: longo@mat.uniroma2.it, \\ roberts@mat.uniroma2.it \\ *** Institut für Theoretische Physik, \\ Universität Göttingen, \\ D-37073 Göttingen, Germany \\ e-mail: verch@theorie.physik.uni-goettingen.de
}

\begin{abstract}
The first part of this paper extends the Doplicher-Haag-Roberts theory of superselection sectors to quantum field theory on arbitrary globally hyperbolic spacetimes. The statistics of a superselection sector may be defined as in flat spacetime and each charge has a conjugate charge when the spacetime possesses non-compact Cauchy surfaces. In this case, the field net and the gauge group can be constructed as in Minkowski spacetime.

The second part of this paper derives spin-statistics theorems on spacetimes with appropriate symmetries. Two situations are considered: First, if the spacetime has a bifurcate Killing horizon, as is the case in the presence of black holes, then restricting the observables to the Killing horizon together with "modular covariance" for the Killing flow yields a conformally covariant quantum field theory on the circle and a conformal spin-statistics theorem for charged sectors localizable on the Killing horizon. Secondly, if the spacetime has a rotation and PT symmetry like the Schwarzschild-Kruskal black holes, "geometric modular action" of the rotational symmetry leads to a spin-statistics theorem for charged covariant sectors where the spin is defined via the $S U(2)$-covering of the spatial rotation group $S O(3)$.
\end{abstract}

\footnotetext{
${ }^{1}$ Supported by GNAFA and MURST.
} 


\section{Table of Contents}

1. Introduction 2

1.1 General Setting 3

1.2 Superselection Sectors 5

1.3 Covariant Sectors and Univalence (Spin) 7

1.4 Tomita-Takesaki Theory and Symmetry $\quad 7$

1.5 Modular Inclusion and Conformal Theories on the Circle 8

1.6 Description of Contents 9

2. Some Spacetime Geometry 10

2.1 Generalities 10

2.2 Appendix to Chapter $2 \quad 14$

3. Superselection Structure in Curved Spacetimes $\quad 15$

3.1 Introduction 15

3.2 The Selection Criterion 17

3.3 Localized Endomorphisms 18

3.4 The Left Inverse and Charge Transfer 23

3.5 Sectors of a Fixed-Point Net 25

3.6 Appendix to Chapter $3 \quad 27$

4. The Conformal Spin and Statistics Relation

for Spacetimes with Bifucate Killing Horizon 37

4.1 Spacetimes with bKh 38

4.2 Conformal Spin-Statistics Relation 42

4.3 Appendix to Chapter $4 \quad 49$

5. The Spin and Statistics Relation for Spacetimes with Rotation Symmetry 51

5.1 Geometric Assumptions 51

5.2 Quantum Field Theories on Spacetimes with Rotation Symmetry 56

5.3 Appendix. Equivalence between local and global intertwiners in Minkowski spacetime 65

$\begin{array}{ll}\text { Acknowledgements } & 68\end{array}$

$\begin{array}{ll}\text { References } & 69\end{array}$

\section{Introduction.}

General Relativity is a theory of gravitation with a geometric interpretation. A solution to the Einstein-Hilbert equations describes a curved spacetime manifold, whose curvature is related to the distribution of matter.

Quantum Field Theory on the other hand arose as a theory for describing finitely many elementary particles and the underlying mathematical structure is that of a net of noncommutative von Neumann algebras of local observables.

There have been many attempts to fuse the two theories to obtain a theory of Quantum Gravity but, as is well known, the basic problems remain unsolved and their solution would seem to be still a long way off.

There is however one theory describing the effects of gravitation on quantum 
systems and this is Quantum Field Theory on a Curved Spacetime, where the gravitational field is treated as a background field so that the backreaction of the quantum system is ignored. Of course, this approximation cannot be expected to remain valid down to distances comparable to the Planck length.

Progress in the field was initially hampered not only by the difficulties of handling interactions, well known from Minkowski space, but also through using a mathematical formalism which was not general enough. Nor did it help that no really interesting physical effects were found. This last point changed dramatically with the advent of Black Hole Thermodynamics and more particularly with the well known Hawking effect whereby a quantum effect causes a black hole to radiate thermally [35, 62].

More recently, the field has evolved rapidly on the mathematical side, too, primarily thanks to adopting methods and concepts from algebraic quantum field theory as e.g. in the work of [27, 40, 34, 46, 62]. But there have been other important developments, too. In particular, the discovery by Radzikowski that the Hadamard condition is equivalent to a wavefront set condition [50, 13] is worth mentioning. This has led to ambitious rigorous work on perturbative quantum field theory in curved spacetime by Brunetti and Fredenhagen [12]. Very recent work in algebraic quantum field theory [19, 51] contributes to clarifying the structure of quantum field theories on anti-de Sitter spacetime and its conformal boundary, an issue which has nowadays attracted great attention.

The DHR analysis of superselection sectors in Minkowski spacetime is a good illustration of the effectiveness of algebraic quantum field theory in treating structural and conceptual problems. The aim of this paper is to lay the foundations of superselection theory in quantum field theory on curved spacetimes and to derive some first results.

We find it advantageous to proceed by recalling, for the benefit of the non-expert reader, the basic ideas and features of algebraic quantum field theory relevant to the two main themes of this paper: the general theory of superselection selection sectors and the connection between Tomita-Takesaki modular theory of von Neumann algebras and spacetime symmetries, particularly in the context of covariant superselection sectors. Our presentation will be simplified, with full details appearing in the main body of the paper. Readers familiar with superselection theory and the relations between modular theory and symmetry in algebraic quantum field theory may wish to turn directly to the outline of the contents in Sec. 1.6 where relations to other papers are indicated.

\subsection{Algebraic Quantum Field Theory on Curved Space- times: General Setting}

In formulating algebraic quantum field theory on a curved spacetime one assumes the underlying spacetime to be described by a smooth manifold $M$ (of any dimension $\geq 2$ ) together with a Lorentzian metric $g$. The quantum system in question is supposed to be described by an inclusion preserving map $\mathcal{K} \ni \mathcal{O} \mapsto \mathcal{A}(\mathcal{O})$ assigning to each member $\mathcal{O}$ in a collection $\mathcal{K}$ of subregions of $M$ a $C^{*}$-algebra $\mathcal{A}(\mathcal{O})$. Usually, $\mathcal{K}$ is chosen to be a base for the topology of $M$ (we will specify $\mathcal{K}$ later on). 
The motivating idea is that $\mathcal{A}(\mathcal{O})$ contains the observables which can be measured at times and locations within the spacetime region $\mathcal{O}$ and that the way these algebras relate to each other for different regions $\mathcal{O}$ essentially fixes the physical content of the theory 34

The collection $\mathcal{K}$ of subregions of $M$ need not be directed under inclusion, but we shall nevertheless refer to $\mathcal{K} \ni \mathcal{O} \mapsto \mathcal{A}(\mathcal{O})$ as a net of local algebras. If $\mathcal{K}$ is directed, then one can form the "quasilocal algebra", i.e. the smallest $C^{*}$-algebra containing all the local algebras $\mathcal{A}(\mathcal{O})$. It is the norm closure of the union of the local algebras, $\overline{\bigcup_{\mathcal{O}} \mathcal{A}(\mathcal{O})}$. In the generic case where $\mathcal{K}$ is not directed, this possibility is denied to us. But one can still expect Hilbert space representations of the inclusion-preserving map $\mathcal{K} \ni \mathcal{O} \mapsto \mathcal{A}(\mathcal{O})$. More precisely, we say that a representation of $\mathcal{K} \ni \mathcal{O} \mapsto \mathcal{A}(\mathcal{O})$ is a consistent family $\left\{\pi_{\mathcal{O}}\right\}_{\mathcal{O} \in \mathcal{K}}$ of representations of the local algebra $\mathcal{A}(\mathcal{O})$ by bounded operators on a common Hilbert space $\mathcal{H}^{\pi}$, i.e. $\pi_{\mathcal{O}_{1}}\left\lceil\mathcal{A}(\mathcal{O})=\pi_{\mathcal{O}}\right.$ whenever $\mathcal{O}_{1} \supset \mathcal{O}$.

For the known examples of quantum field theories on globally hyperbolic spacetimes and (conformal) quantum field theories on $S^{1}$, such representations exist in abundance. (There are indications to the contrary for non-globally-hyperbolic spacetimes 39, 38. The present paper is restricted to quantum field theory on globally hyperbolic spacetimes and the above notion of representation suffices.) Every representation $\left\{\pi_{\mathcal{O}}\right\}_{\mathcal{O} \in \mathcal{K}}$ yields states on the local algebras $\mathcal{A}(\mathcal{O})$ since each normal state $\omega$ on $\mathcal{B}\left(\mathcal{H}^{\pi}\right)$ restricts to a state

$$
\omega_{\mathcal{O}}(A):=\omega\left(\pi_{\mathcal{O}}(A)\right), \quad A \in \mathcal{A}(\mathcal{O})
$$

of the local algebra. Not every consistent family of local states corresponds to a physical state of the system; nor can all representations of the observable net be considered as physical so one needs criteria to select physical representations. In practice, one begins with some collection of physical representations and uses them to construct others. In what follows, we compile a brief list of criteria to be fulfilled by such an initial collection $\mathfrak{P}$ of physical representations of the net $\mathcal{K} \ni \mathcal{O} \mapsto \mathcal{A}(\mathcal{O})$ of local observables on a curved spacetime $(M, g)$.

1) $\pi_{\mathcal{O}}, \mathcal{O} \in \mathcal{K}$ is faithful for each $\left\{\pi_{\mathcal{O}}\right\}_{\mathcal{O} \in \mathcal{K}} \in \mathfrak{P}$. Otherwise the description of the system by the net of local algebras $\mathcal{K} \in \mathcal{O} \mapsto \mathcal{A}(\mathcal{O})$ would contain redundancies.

2) Locality: The algebras $\pi_{\mathcal{O}}(\mathcal{A}(\mathcal{O}))$ and $\pi_{\mathcal{O}^{\prime}}\left(\mathcal{A}\left(\mathcal{O}^{\prime}\right)\right)$ commute elementwise if the regions $\mathcal{O}$ and $\mathcal{O}^{\prime}$ cannot be connected by a causal curve.

3) Irreducibility and Duality: $\mathfrak{P}$ consists of irreducible representations, i.e. representations $\left\{\pi_{\mathcal{O}}\right\}_{\mathcal{O} \in \mathcal{K}}$ fulfilling 甘

$$
\left\{\bigcup_{\mathcal{O} \in \mathcal{K}} \pi_{\mathcal{O}}(\mathcal{A}(\mathcal{O}))\right\}^{\prime}=\mathbb{C} 1
$$

These representations are required to fulfill essential duality, i.e. the net,

$$
\mathcal{K} \ni \mathcal{O} \mapsto \mathcal{A}_{\pi}^{d}(\mathcal{O}):=\bigcap_{\mathcal{O}_{1}} \pi_{\mathcal{O}_{1}}\left(\mathcal{A}\left(\mathcal{O}_{1}\right)\right)^{\prime}
$$

\footnotetext{
${ }^{1} \mathcal{A}^{\prime}=\{B \in \mathcal{B}(\mathcal{H}): B A=A B \forall A \in \mathcal{A}\}$ denotes the commutant of $\mathcal{A} \subset \mathcal{B}(\mathcal{H})$.
} 
is local, where the intersection is taken over all $\mathcal{O}_{1} \in \mathcal{K}$ causally disjoint from $\mathcal{O}$.

This property is stronger than locality but not as strong as Haag duality which demands that $\pi_{\mathcal{O}}(\mathcal{A}(\mathcal{O}))^{\prime \prime}=\mathcal{A}_{\pi}^{d}(\mathcal{O})$ for all $\mathcal{O} \in \mathcal{K}$. This latter property means that the von Neumann algebras $\pi_{\mathcal{O}}(\mathcal{A}(\mathcal{O}))^{\prime \prime}$ cannot be enlarged by adding elements of $\mathcal{B}\left(\mathcal{H}^{\pi}\right)$ without violating the locality condition.

4) Local Equivalence: Whenever $\left\{\pi_{\mathcal{O}}\right\}_{\mathcal{O} \in \mathcal{K}}$ and $\left\{\pi_{\mathcal{O}}^{\prime}\right\}_{\mathcal{O} \in \mathcal{K}}$ are two members of $\mathfrak{P}$, there is for each $\mathcal{O} \in \mathcal{K}$ a unitary $U_{\mathcal{O}}: \mathcal{H}^{\pi} \rightarrow \mathcal{H}^{\pi^{\prime}}$ such that

$$
U_{\mathcal{O}} \pi_{\mathcal{O}}(A)=\pi_{\mathcal{O}}^{\prime}(A) U_{\mathcal{O}}, \quad A \in \mathcal{A}(\mathcal{O})
$$

5) Covariance: For each $\left\{\pi_{\mathcal{O}}\right\}_{\mathcal{O} \in \mathcal{K}} \in \mathfrak{P}$ there is an (anti-) unitary $G \ni \gamma \mapsto U_{\pi}(\gamma)$ of a (subgroup of) the spacetime isometry group $G$ on $\mathcal{H}^{\pi}$ so that

$$
U_{\pi}(\gamma) \pi_{\mathcal{O}}(\mathcal{A}(\mathcal{O})) U_{\pi}(\gamma)^{*}=\pi_{\gamma \mathcal{O}}(\mathcal{A}(\gamma \mathcal{O})), \quad \gamma \in G, \mathcal{O} \in \mathcal{K}
$$

Obviously, if the underlying spacetime $(M, g)$ has a trivial isometry group, this condition is void.

If $(M, g)$ is Minkowski spacetime, there is typically a distinguished vacuum representation $\pi_{\text {vac }}$ in $\mathfrak{P}$ which is irreducible and covariant and possesses a cyclic vacuum vector $\Omega_{\mathrm{vac}} \in \mathcal{H}^{\pi_{\mathrm{vac}}}$ invariant under the action of $U_{\pi_{\mathrm{vac}}}$. Moreover, a vacuum representation fulfills the spectrum condition, i.e. the time-translations in any Lorentz frame have positive generator. In more general spacetimes, one can usually not select a distinguished vacuum representation by similar requirements since, in the absence of a sufficiently large isometry group, there is no analogue of the vacuum vector nor of the spectrum condition. However, one expects that a collection of physical representations $\mathfrak{P}$ can still be selected in quantum field theory on curved spacetimes, even if there is no single preferred representation. For a Klein-Gordon field on any four-dimensional globally hyperbolic spacetime the representations induced by pure quasifree Hadamard states have been shown to form a collection $\mathfrak{P}$ satisfying the conditions listed above 59.

\subsection{Superselection Sectors}

We assume now that a curved spacetime $(M, g)$, a net $\mathcal{K} \ni \mathcal{O} \mapsto \mathcal{A}(\mathcal{O})$ of local algebras on this spacetime background and a collection $\mathfrak{P}$ of physical representations fulfilling the conditions stated above has been given. To simplify notation, we denote a representation $\left\{\pi_{\mathcal{O}}\right\}_{\mathcal{O} \in \mathcal{K}}$ of the net of local algebras simply by $\pi$.

Picking an irreducible physical representation $\pi^{0} \in \mathfrak{P}$ as reference, another irreducible representation $\pi$ (not necessarily belonging to $\mathfrak{P}$ ) is said to satisfy the selection criterion for localizable charges if, given $\mathcal{O} \in \mathcal{K}$, there is a unitary $V_{\mathcal{O}}$ between the representation Hilbert spaces $\mathcal{H}^{\pi}$ and $\mathcal{H}^{\pi^{0}}$ such that

$$
V_{\mathcal{O}} \pi_{\mathcal{O}_{1}}(A)=\pi_{\mathcal{O}_{1}}^{0}(A) V_{\mathcal{O}}, \quad A \in \mathcal{A}\left(\mathcal{O}_{1}\right),
$$

\footnotetext{
${ }^{2}$ That is, $U_{\pi}(\gamma)$ is anti-unitary if $\gamma$ reverses the time-orientation, otherwise it is unitary
} 
for all regions $\mathcal{O}_{1} \in \mathcal{K}$ causally disjoint from $\mathcal{O}$. Irreducible representations which fulfill this selection criterion and are globally unitarily equivalent are said to carry the same charge, or to define the same superselection sector.

The selection criterion thus selects representations $\pi$ differing from the reference representation by some "charges" which can be localized in any spacetime region $\mathcal{O}$ (and are then not detectable in spacetime regions situated acausally to $\mathcal{O}$ ). This form of localizability does not apply to all kinds of charges, e.g. electric charge is not localizable in this way (cf. [34] and references therein for further discussion). Yet for certain general types of charges, like flavours in strong interactions, this description is appropriate and hence a useful starting point.

The notion of localized charge and superselection sector now apparently depends on the chosen reference representation $\pi^{0}$ (typically the vacuum representation in the case of flat spacetime), but as physical representations are required to be locally equivalent, the charge structure, being given by the structure of the space of intertwining operators of representations fulfilling the selection criterion, is expected to be independent of that choice. Here, a bounded operator $T: \mathcal{H}^{\pi} \rightarrow \mathcal{H}^{\pi^{\prime}}$ is called an intertwiner for the representations $\pi$ and $\pi^{\prime}$ of $\mathcal{K} \ni \mathcal{O} \mapsto \mathcal{A}(\mathcal{O})$ if

$$
T \pi_{\mathcal{O}}(A)=\pi_{\mathcal{O}}^{\prime}(A) T, \quad A \in \mathcal{A}(\mathcal{O}), \quad \mathcal{O} \in \mathcal{K} .
$$

A crucial point is that the space of intertwiners admits a product having the formal properties of a tensor product. The statistics of the charges in the theory reflects the behaviour of this product under interchange of factors. Under certain general conditions, e.g. if the Cauchy surfaces of the spacetime are not compact, each charge has a conjugate charge and then the statistics of each charge can be characterized by a number, its statistics parameter. This number can be split into its phase and modulus being, respectively, the statistics phase and the inverse of the statistical dimension. (The latter is defined to be $\infty$ if the statistics parameter equals 0 and one says that the superselection sector has infinite statistics. We shall only consider superselection sectors having finite statistics.) If the statistics phase takes the values \pm 1 , then the (para- )Bose/Fermi alternative holds in that there is a conventional description in terms of Bose and Fermi fields commuting or anticommuting when localized in causally disjoint regions. This is the generic situation in physical spacetime dimension. In lower spacetime dimension, braid group statistics may occur and the statistics phase may take values different from \pm 1 .

In previous papers 25, 24] it was shown that, in Minkowski spacetime, one can construct a field net together with a unitary action of a compact (global) gauge group containing the observable net $\mathcal{A}$ as fixed points so that the superselection sectors correspond naturally to the equivalence classes of irreducible representations of the gauge group. A similar result will turn out to hold in curved spacetime as well. As so little input is used (essentially only the physically motivated selection criterion and local commutativity of the observables) this result clearly demonstrates how effective the operator algebraic approach to quantum field theory can be. 


\subsection{Covariant Sectors and Univalence (Spin)}

Our notion of spin on curved spacetime involves a group $G$ of isometries although there ought to be a more general notion not involving symmetries. For this reason, we assume covariance of our reference representation $\pi^{0}$.

A superselection sector described by a representation $\pi$ is covariant if there exists an (anti-) unitary representation $\widetilde{G} \ni \Gamma \mapsto \widetilde{U}_{\pi}(\Gamma)$ of the universal covering group of $G$ on $\mathcal{H}^{\pi}$ with

$$
\pi_{\gamma \mathcal{O}} \circ \alpha_{\gamma}=\operatorname{Ad} \widetilde{U}_{\pi}(\Gamma) \circ \pi_{\mathcal{O}}, \quad \Gamma \in \widetilde{G}, \quad \mathcal{O} \in \mathcal{K},
$$

where $\Gamma \mapsto \gamma$ denotes the covering projection.

We may now consider continuous curves $[0,2 \pi] \ni t \mapsto \Gamma(t)$ whose projection $[0,2 \pi] \ni t \mapsto \gamma(t)$ is a cycle, i.e. a closed curve possessing no closed sub-curves. $\widetilde{U}_{\pi}(\Gamma(2 \pi))$ may be different from 1 , but as $\pi$ is irreducible, $\widetilde{U}_{\pi}(\Gamma(2 \pi))=s_{\pi} \cdot 1$ where $s_{\pi}$ is a complex number of modulus 1 . When the cycle $\gamma([0,2 \pi])$ has the geometric interpretation of a "spatial rotation by $2 \pi$ ", then it is appropriate to refer to the phase factor $s_{\pi}$ as the "spin", or more precisely, the univalence of the charge represented by $\pi$. I Then, the spin-statistics connection is said to hold if, for all covariant superselection sectors of the theory, the univalence equals the statistics phase.

\subsection{Tomita-Takesaki Theory and Symmetry}

Let us next summarize some basic points of the modular theory for von Neumann algebras by Tomita and Takesaki [58]. Given a von Neumann algebra $\mathcal{N}$ on a Hilbert space $\mathcal{H}$ together with a cyclic and separating unit vector $\Omega \in \mathcal{H}$, the antilinear operator $S: \mathcal{N} \Omega \rightarrow \mathcal{N} \Omega$ defined by $S(A \Omega):=A^{*} \Omega$ admits a minimal closed extension with polar decomposition $\bar{S}=J \Delta^{1 / 2}$ where $J$ is anti-unitary. $J$ is referred to as modular conjugation and $\left\{\Delta^{i t}\right\}_{t \in \mathbb{R}}$ as modular unitary group associated with the pair $\mathcal{N}, \Omega$; one refers to $\mathrm{Ad} J$ as the antilinear modular morphism associated with $\mathcal{N}, \Omega$ and usually denotes it by $j$. These modular objects satisfy $J \mathcal{N} J=\mathcal{N}^{\prime}$ and $\Delta^{i t} \mathcal{N} \Delta^{-i t}=\mathcal{N}$, $t \in \mathbb{R}$. Moreover, a state $\omega$ on a $C^{*}$-algebra $\mathfrak{A}$ is a KMS-state (thermal equilibrium state) at inverse temperature $\beta$ with respect to a one-parametric group $\left\{\alpha_{t}\right\}_{t \in \mathbb{R}}$ of modular automorphisms of $\mathcal{A}$ if and only if

$$
\pi_{\omega} \circ \alpha_{t}=\operatorname{Ad} \Delta^{-i \beta t / 2 \pi} \circ \pi_{\omega}
$$

where $\pi_{\omega}$ is the GNS-representation of $\omega$ and $\left\{\Delta^{i t}\right\}_{t \in \mathbb{R}}$ is the modular group of $\pi_{\omega}(\mathcal{A})^{\prime \prime}, \Omega_{\omega}, \Omega_{\omega}$ being the GNS-vector. Thus the modular group may, in certain situations, have a physical (dynamical) significance.

Furthermore, Bisognano and Wichmann showed [4] that, in Wightman's setting of quantum fields in Minkowski spacetime, the modular objects associated with pairs $\mathcal{A}(W), \Omega$, where $\mathcal{A}(W)$ is the von Neumann algebra of observables in a certain "wedge-

\footnotetext{
${ }^{3}$ We do not wish to discuss how $s_{\pi}$ depends on the different possible "rotations". It suffice to say that in the relevant cases the above procedure assigns an invariant $s_{\pi}$ to any covariant superselection sector.
} 
region" f $W$ and $\Omega$ the vacuum vector, induce spacetime transformations. That is, if $J_{W},\left\{\Delta_{W}^{i t}\right\}_{t \in \mathbb{R}}$ denote the corresponding modular objects, then there are elements $\mathbf{j}_{W}, \Lambda_{W, t}$ in the Poincaré group so that

$$
\begin{aligned}
\operatorname{Ad} J_{W} \mathcal{A}(\mathcal{O}) & =\alpha_{\mathbf{j}_{W}}(\mathcal{A}(\mathcal{O}))=\mathcal{A}\left(\mathbf{j}_{W}(\mathcal{O})\right) \\
\operatorname{Ad} \Delta_{W}^{i t} \mathcal{A}(\mathcal{O}) & =\alpha_{\Lambda_{W, t}}(\mathcal{A}(\mathcal{O}))=\mathcal{A}\left(\Lambda_{W, t}(\mathcal{O})\right),
\end{aligned}
$$

for all open subregions $\mathcal{O}$ of Minkowski spacetime, all $t \in \mathbb{R}$ and all wedge-regions $W$.

Further investigations (e.g. [5, 30, 17, 15, 8]) relate spacetime symmetries and modular objects and indicate that vacuum states in Minkowski spacetime can possibly be characterized through the geometric meaning of the modular objects associated with $\mathcal{A}(W), \Omega$ for a certain class of wedge-regions $W$. This idea has been pursued in non-flat spacetimes with a sufficiently rich group of isometries and a suitable class of wedge-regions, such as de Sitter spacetime and, to some extent, Schwarzschild-Kruskal spacetime, too [56, 10, 9]. There are indications that physical states of quantum field theory on arbitrary spacetime manifolds can be distinguished by the "geometrical action" of the corresponding modular objects for a certain class of regions, understood in sufficient generality. The reader is referred to [18 and references therein for considerable further discussion.

In Minkowski spacetime, the geometric action of the modular objects associated with wedge-algebras $\mathcal{A}(W)$ and the vacuum vector $\Omega$ has important consequences for the relation between spin and statistics. It can be derived either from geometric modular action [42], i.e. the geometric action of the modular conjugations as in (1.1), or from modular covariance [31], meaning the geometric action of the modular group as in (1.2). Similarly, for conformal quantum field theories on the circle $S^{1}$ where modular objects and conformal symmetry are intimately related, there is a spinstatistic relation, as will be briefly summarized in the next section.

\subsection{Modular Inclusion and Conformal Theories on the Circle}

In this section we summarize the connection between conformally covariant theories on the circle $S^{1}$ and halfsided modular inclusions established by Wiesbrock 64, 65, 66, 67.

We briefly recall what is meant by a conformally covariant theory on the circle $S^{1}$ (see e.g. [30, 32] for further details). This is a net (or precosheaf) $I \mapsto \mathcal{M}(I)$ taking proper open subintervals $I$ of $S^{1}$ to von Neumann algebras $\mathcal{M}(I)$ on a Hilbert space $\mathcal{H}_{\mathcal{M}}$ so that locality holds, i.e. $\mathcal{M}\left(S^{1} \backslash \bar{I}\right) \subset \mathcal{M}(I)^{\prime}$. Moreover, there exists a unitary strongly continuous positive energy representation $U$ of $P S L(2, \mathbb{R})$ acting covariantly, $U(g) \mathcal{M}(I) U(g)^{*}=\mathcal{M}(g I)$, and preserving a unit vector $\Omega_{\mathcal{M}}$, cyclic for the von Neumann algebra generated by the $\mathcal{M}(I)$ 's. (In other words, the theory is given in a reference "vacuum representation".)

The theory may be equivalently described as a net of von Neumann algebras indexed by intervals on the real line, identified as the circle with one point removed.

\footnotetext{
${ }^{4} \mathrm{~A}$ wedge region is any Poincaré transform of the set $\left\{\left(x_{0}, \ldots, x_{n}\right): 0<x_{1}, 0 \leq\left|x_{0}\right|<x_{1}\right\}$ in Minkowski spacetime.
} 
Using the Cayley transform, conformal transformations on the circle correspond to fractional linear transformations on the line. Modular transformations have a geometric meaning and Haag duality holds for any conformal theory on the circle, namely $\mathcal{M}\left(S^{1} \backslash \bar{I}\right)=\mathcal{M}(I)^{\prime}$ [14. Haag duality on the line, $\mathcal{M}(\mathbb{R} \backslash \bar{I})=\mathcal{M}(I)^{\prime}$, holds precisely when the net $I \mapsto \mathcal{M}(I)$ is strongly additive[33], i.e. if $\mathcal{M}(I)=\mathcal{M}\left(I_{1}\right) \vee \mathcal{M}\left(I_{2}\right)$ whenever the union of $I_{1}$ and $I_{2}$ yields $I$ up to at most a single point.

We recall that a \pm hsm inclusion $(\mathcal{N} \subset \mathcal{N}, \Omega)$ is given by a pair $\mathcal{N} \subset \mathcal{M}$ of von Neumann algebras on some Hilbert space together with a unit vector $\Omega$, cyclic and separating for both $\mathcal{N}$ and $\mathcal{M}$, such that $\Delta^{i t} \mathcal{N} \Delta^{-i t} \subset \mathcal{N}$ for all $\mp t \geq 0$, where $\Delta^{i t}$, $t \in \mathbb{R}$, is the modular group of $\mathcal{M}, \Omega$. A \pm hsm inclusion $(\mathcal{N} \subset \mathcal{M}, \Omega)$ is called standard if $\Omega$ is cyclic for $\mathcal{N}^{\prime} \cap \mathcal{M}$, too (hsm abbreviates "half sided modular").

An interesting result of Wiesbrock ( 64, 65] see also [33]) asserts that there is a one-to-one correspondence between strongly additive conformally covariant theories on $S^{1}$ and standard \pm hsm inclusions.

The rotations of $S^{1}$ form a subgroup of the covering group of $P S L(2, \mathbb{R})$. Let $\pi$ be a Hilbert space representation of a covariant superselection sector and $\widetilde{U}_{\pi}$ the associated unitary representation of the covering group of $P S L(2, \mathbb{R})$. Assuming that $\widetilde{U}_{\pi}$ has positive energy, the generator of rotations in the unitary representation $\widetilde{U}_{\pi}$ has a lowest eigenvalue $L_{\pi}$. Then the conformal spin of the superselection sector, or rather, its univalence, is defined by $s_{\pi}=\mathrm{e}^{2 \pi i L_{\pi}}$. For superselection sectors with positive energy in a conformally covariant theory on $S^{1}$, the univalence equals the statistics phase, which may be any complex number of modulus 1 [32].

\subsection{Description of Contents}

We now describe the contents of the subsequent chapters.

In Chapter 2 we summarize several notions of spacetime geometry needed here. Lemma 2.2, of relevance to superselection theory, asserts that the set of pairs of causally separated points in a globally hyperbolic spacetime is connected.

Chapter 3 contains the general framework for superselection theory in curved spacetimes, patterned conceptually on the DHR analysis in Minkowski spacetime (23], cf. also [34, 53 and references given there). It will be formulated for nets $\mathcal{K} \ni \mathcal{O} \mapsto \mathcal{A}(\mathcal{O})$ of operator algebras in a reference representation with general index sets $\mathcal{K}$ possessing a partial ordering and a causal disjointness relation. Thus quantum fields on arbitrary globally hyperbolic spacetimes in any dimensions, with compact or non-compact Cauchy surfaces, as well as quantum field theory on the circle, can be treated on an equal footing. The existence of statistics is established in this generality. If the index set $\mathcal{K}$ is directed, all the other basic results known for superselection theory on Minkowski spacetime, classification of statistics, existence of charge conjugation and construction of field algebra and gauge group (cf. [25]) can again be shown to hold.

Chapter 4 begins with a summary of the geometry of spacetimes with a bifurcate Killing horizon following Kay and Wald 40]. We introduce a family of wedge-regions $R_{a}, a>0$ which are copies of the canonical right wedge shifted by $a$ in the affine geodesic parameter on the horizon (a similar construction can be carried out for the 
left wedge). We suppose that we are given a net of von Neumann algebras $\mathcal{O} \mapsto \mathcal{A}(\mathcal{O})$ in the representation of a state which is, in restriction to the subnet of observables which are localized on the horizon, a KMS-state at Hawking temperature for the Killing flow. Thus on the horizon we have modular covariance and are consequently in Wiesbrock's situation of half-sided modular inclusion [64. Using Haag duality and additivity of the net, it follows that the maximal subnet of observables localized on the horizon is a conformally covariant family of von Neumann algebras. Restricting the original net of von Neumann algebras to the Killing horizon thus yields a conformal quantum field theory on $S^{1}$. A conformal spin is therefore assigned to a superselection sector of the original theory, localizable on the horizon, and the conformal spin-statistics connection [32] holds. This approach has, however, the drawback of applying only to horizon-localizable charges, and this may be quite restrictive.

In Chapter 5 we introduce a class of spacetimes with a special rotation symmetry and certain adapted wedge-regions. Essentially we assume that there is a group of symmetries, to be viewed as rotations, generated by pairs of time-reversing wedgereflections mapping wedge-regions onto each other. In the Schwarzschild-Kruskal spacetime, for example, these wedge-regions can be envisaged as the causal completions of "halves" of the canonical Cauchy-surfaces chosen so that rotating by $\pi$ about a suitable axis maps each such half onto its causal complement. These wedge-regions differ from the usual canonical "right" and "left" wedges $(R$ and $L$ in Chapter 4$)$ and lie in a sense transversal to the latter. Then we consider a net of von Neumann algebras $\mathcal{O} \mapsto \mathcal{A}(\mathcal{O})$ over such a spacetime in a representation where the full isometry group acts covariantly. Moreover we suppose that there is an isometry-invariant state and that the modular conjugations associated with the vacuum vector and the von Neumann algebras $\mathcal{A}(W)$ for the said class of wedges $W$ induce the geometric action of the wedge-reflections. This form of geometric modular action will allow us to define the rotational spin of a covariant superselection sector and to derive the spin and statistics connection using a variant of arguments presented in 445.

\section{Some Spacetime Geometry}

\subsection{Generalities}

In the present section we summarize some notions about causal structure of Lorentzian manifolds, thereby establishing our notation. Standard references for this section include [3, 36, 49, 61].

We begin by recalling that a curved spacetime $(M, g)$ is a $1+s$-dimensional $(s \in \mathbb{N})$, Lorentzian manifold. In other words, it is a $1+s$-dimensional orientable, Hausdorff, second countable $C^{\infty}$-manifold equipped with a smooth Lorentzian metric $g$ having signature $(+,-, \ldots,-)$.

A continuous, (piecewise) smooth curve $\gamma: I \rightarrow M$, defined on a connected subset $I$ of $\mathbb{R}$ and having tangent $\dot{\gamma}$, is called a timelike curve whenever $g(\dot{\gamma}, \dot{\gamma})>0$, a causal curve if $g(\dot{\gamma}, \dot{\gamma}) \geq 0$, and a lightlike curve if $g(\dot{\gamma}, \dot{\gamma})=0$ while $\dot{\gamma} \neq 0$, for all parameter values $t$. 
A spacetime $(M, g)$ is called time-orientable if there exists a global timelike (nonvanishing) vectorfield $\xi$ on $M$. Such a vector field induces a time-orientation: a causal curve $\gamma$ is called future-directed or past-directed according as $g(\xi, \dot{\gamma})>0$ or $g(\xi, \dot{\gamma})<0$. We shall henceforth tacitly assume our spacetimes to be time-orientable with a given time-orientation.

A future-directed causal curve $\gamma: I \rightarrow M$ is said to have a future (past)-endpoint if $\gamma(t)$ converges to some point in $M$ as the parameter $t$ approaches sup $I(\inf I$ ). Correspondingly one defines the past (future)-endpoints of past-directed causal curves. A future (past)-directed causal curve is said to start at a point $p \in M$ provided that $p$ is the past (future)-endpoint of $\gamma$. Moreover, one calls a future (past)-directed causal curve future (past)-inextendible if it possesses no future (past)-endpoint.

For any subset $\mathcal{O}$ of $M$ one defines the sets $J^{ \pm}(\mathcal{O})$ as consisting of all points in $M$ lying on future(+)/past(-)-directed causal curves that start at some point in $\mathcal{O}$. Then $J^{ \pm}(\mathcal{O})$ are called the causal future $(+) /$ causal past $(-)$ of $\mathcal{O}$. The set $J(\mathcal{O}):=J^{+}(\mathcal{O}) \cup J^{-}(\mathcal{O})$ is then referred to as the causal set of $\mathcal{O}$. The subsets $D^{ \pm}(\mathcal{O})$ of $M$ are, for given $\mathcal{O} \subset M$, defined as the collection of all those points $p \in M$ such that every past(+)/future(-)-inextendible causal curve starting at $p$ meets $\mathcal{O}$. One calls $D^{ \pm}(\mathcal{O})$ the future $(+) /$ past(-)-domain of dependence of $\mathcal{O}$, and $D(\mathcal{O}):=D^{+}(\mathcal{O}) \cup D^{-}(\mathcal{O})$ the domain of dependence of $\mathcal{O}$.

One says that two points $p$ and $q$ in $M$ are causally disjoint, in symbols $p \perp q$, if there are open neighbourhoods $U$ of $p$ and $V$ of $q$ such that there is no causal curve connecting $U$ and $V$ (i.e. $U \cap J(V)=\emptyset=V \cap J(U)$ ). Correspondingly one calls two subsets $P$ and $Q$ of $M$ causally disjoint if $p \perp q$ holds for all pairs $p \in P$ and $q \in Q$; this will be abbreviated as $P \perp Q$.

In the present paper we will primarily be interested in globally hyperbolic spacetimes. A spacetime $(M, g)$ is globally hyperbolic if it can be smoothly foliated in acausal Cauchy surfaces. Here, an acausal Cauchy surface $C$ is a smooth hypersurface in $M$ such that each causal curve in $(M, g)$ without endpoints meets $C$ exactly once. This implies that $C$ is indeed acausal, i.e. $p \perp q$ holds for all distinct $p, q \in C$. By a (smooth) foliation of $(M, g)$ in acausal Cauchy surfaces we mean a diffeomorphism $F: \mathbb{R} \times \Xi \rightarrow M$ where $\Xi$ is an $s$-dimensional smooth manifold such that $F(\{t\} \times \Xi)$ is, for each $t \in \mathbb{R}$, an acausal Cauchy surface in $(M, g)$, and the curves $\mathbb{R} \ni t \mapsto F(t, q), q \in \Xi$, are timelike and endpointless. Thus, the foliation-parameter $t$ plays the role of a "time-parameter". One may give a broader definition of Cauchy surfaces which are not necessarily acausal, by defining a Cauchy surface as a $C^{0}$ hypersurface $C$ such that $C \cap$ int $J^{ \pm}(C)=\emptyset$ and $D(C)=M$. With this definition, a Cauchy surface is allowed to have lightlike parts. Such a broader definition of Cauchy surfaces is often useful. However, it is a remarkable fact that the existence of a single, not necessarily acausal Cauchy surface in $(M, g)$ already implies that $(M, g)$ is globally hyperbolic in the above sense [29, 21, 61].

Whilst the question of whether physical spacetime models are necessarily globally hyperbolic has been discussed in the literature (see [20, 61, 63] and references given there), it is certainly the case that a great number of the prominent spacetime models are globally hyperbolic, like Schwarzschild-Kruskal, deSitter, the Robertson-Walker models, and many others, including of course Minkowski spacetime. One may there- 
fore regard the class of globally hyperbolic spacetimes as being sufficiently general and comprising many examples of physical interest. Note that global hyperbolicity in no way presupposes the presence of spacetime symmetries.

At this point we recall some properties of causal sets; for their proof and further discussion, we refer to the indicated references. Whenever $N \subset M$ and $(M, g)$ is globally hyperbolic, then: $N$ compact implies $J^{ \pm}(N)$ closed, $N$ compact implies that $J(N) \cap C$ is compact for each Cauchy surface $C, N$ compact implies $D(N)$ compact. Furthermore, $J^{+}\left(N_{+}\right) \cap J^{-}\left(N_{-}\right)$is empty or compact for all compact $N_{+}, N_{-} \subset$ $M$. Moreover, in (time-orientable) spacetimes $(M, g)$, a time-orientation preserving isometry $\tau$ of $(M, g)$ satisfies

$$
\tau\left(J^{ \pm}(\mathcal{O})\right)=J^{ \pm}(\tau(\mathcal{O}))
$$

for $\mathcal{O} \subset M$. It is moreover worth mentioning that for any two subsets $P$ and $Q$ of a globally hyperbolic spacetime $(M, g)$ we have $P \perp Q$ if and only if $P \subset Q^{\perp}$, where the causal complement $Q^{\perp}$ of $Q \subset M$ is defined by $Q^{\perp}:=M \backslash \overline{J(Q)}$, see e.g. [41, Prop. 8.1].

We need to consider special regions of a globally hyperbolic spacetime $(M, g)$ namely those causally closed regions generated by an open subset of a Cauchy surface. More particularly we are interested in regular diamonds defined as follows. A set of the form $\mathcal{O}=\operatorname{int} D(G)$ is a regular diamond provided $\mathcal{O}^{\perp}$ is non-void and

(i) $G$ is an open subset of an acausal Cauchy-surface $C$, and $\bar{G}$ is compact and contractible to a point in $G$,

(ii) $\partial G$, the boundary of $G$, is a (possibly multiply connected) locally flat embedded, two-sided topological submanifold of $C$ which is an embedded $C^{\infty}$-submanifold near to points in each of its connected components.

We refer to [11, 60] for the precise definition of locally flat embeddings and twosidedness. Intuitively, these two conditions are substitutes for the existence of an oriented normal vector field over $\partial G$. These regularity properties serve to prove the following assertion:

Lemma 2.1. Let $\mathcal{O}$ be a regular diamond and $p \in \mathcal{O}^{\perp}$. Then there exists another regular diamond $\mathcal{O}_{1}$ with

$$
\mathcal{O} \cup\{p\} \subset \mathcal{O}_{1}
$$

A rough sketch of the proof will be given in Sec. 2.2, the Appendix to this chapter. The reader is referred to [60] for a detailed proof.

A double cone in Minkowski space is, of course, a regular diamond. Double cones may be generalized easily to curved spacetime. They are sets of the form $\operatorname{int}\left(J^{-}\left(\left\{v^{+}\right\}\right) \cap J^{+}\left(\left\{v^{-}\right\}\right)\right)$with $v^{+} \in \operatorname{int} J^{+}\left(\left\{v^{-}\right\}\right)$. However, double cones need not have the property analogous to Lemma 2.1, think e.g. of a spacelike strip in Minkowski spacetime. Nor is it clear that a double cone is a regular diamond. For this reason, it is not clear, even for simple free fields, whether duality is satisfied for such regions. We expect the requirement of essential duality (cf. Sec. 1.1) to be realistic for regular 
diamonds, in particular, as their bases are assumed contractible. Furthermore, duality for regular diamonds has already been established for the Klein-Gordon field[57] and can presumably be verified for other free fields. For these reasons, we have chosen to use the collection $\mathcal{K}$ of regular diamonds rather than the collection of double cones whose causal complement has non-empty interior as an index set in a globally hyperbolic spacetime.

Given a spacetime $(M, g)$, we introduce the set

$$
X_{M, g}:=\{(x, y) \in M \times M: x \perp y\}
$$

of pairs of causally disjoint points in $M$. According to the definition of causal disjointness, this set is an open subset of $M \times M$. The subsequent assertion about $X_{M, g}$ will prove to be important in discussing the statistics of superselection sectors in the next chapter. It may be known to experts, but as we have not found it in the literature, we put it on record here.

Lemma 2.2. Let $(M, g)$ be a globally hyperbolic spacetime then $X_{M, g}$ is pathwise connected except when its Cauchy surfaces are noncompact and 1-dimensional in which case there are precisely two path-components corresponding to $x$ being causally to the left or to the right of $y$.

Proof. Let $F: \mathbb{R} \times \Xi \rightarrow M$ be a foliation in acausal Cauchy surfaces and write $C:=F(\{0\} \times \Xi)$. We first show that it suffices to restrict one's attention to the Cauchy surface $C$. More precisely, we show that

$$
y:=\{(x, y) \in C \times C: x \perp y\}
$$

is a strong deformation retract of $X_{M, g}$. In fact, using $F$ to parametrize $M$ and defining $h: X_{M, g} \times I \rightarrow X_{M, g}$ by

$$
h\left(t, \xi ; t^{\prime}, \xi^{\prime} ; s\right):=\left((1-s)\left(t+s\left(t^{\prime}-t\right)\right), \xi ;(1-s) t^{\prime}, \xi^{\prime}\right)
$$

we have a homotopy of the identity on $X_{M, g}$ onto the projection, $\left(t, \xi ; t^{\prime}, \xi^{\prime}\right) \mapsto$ $\left(0, \xi ; 0, \xi^{\prime}\right)$, onto $C$ leaving $C$ fixed. The only non-trivial point is to show that the image of $h$ lies in $X_{M, g}$ and this is where the causal structure enters. However, two remarks suffice: first, causal disjointness reduces to disjointness on an acausal Cauchy surface and hence is preserved if we pass from one acausal Cauchy surface to another by changing the value of $t$. Secondly, if we take causally disjoint points $x_{i}=F\left(t_{i}, \xi_{i}\right)$, $i=1,2$ with distinct values of $t$ then the curve $\gamma:\left[\inf \left\{t_{1}, t_{2}\right\}, \sup \left\{t_{1}, t_{2}\right\}\right] \ni t \mapsto$ $F\left(t, \xi_{1}\right)$ is timelike and connects $x_{1}$ with that Cauchy surface of the foliation containing $x_{2}$. Its range must lie in $\left\{x_{2}\right\}^{\perp}$ or there would be a causal curve coming arbitrarily close to connecting $x_{1}$ and $x_{2}$, contrary to assumption. We now know that the inclusion of $y$ in $X_{M, g}$ induces an isomorphism in homotopy and, in particular, an isomorphism of path-components. Now unless $C$ is one dimensional and non-compact, the complement of a point of $C$ is path-connected and $y$ is then also path-connected. If $C$ is one dimensional and non-compact it is isomorphic to $\mathbb{R}$ so that $y$ has two path-components. 
When $X_{M, g}$ has two components, we use the foliation $F: \mathbb{R} \times \mathbb{R} \rightarrow M$ into acausal Cauchy surfaces to distinguish the "right" component from the "left" component as that containing pairs $(x, y)$, where the spatial component of $y$ is greater than that of $x$. In fact, this distinction depends only on the nowhere vanishing spacelike vector field $\xi$ induced by the foliation. Given such a field $\xi$, a spacelike curve $I \ni t \mapsto \gamma(t)$ is called right-directed if $g(\xi, \dot{\gamma})>0$ and left-directed if $g(\xi, \dot{\gamma})<0$ for one and hence all values of $t$. (A different choice of $\xi$ would at most lead to interchanging "right-directed" and "left-directed" since in two spacetime dimensions the set of spacelike vectors at each point has two components.) The orientation of spacelike curves defined in this way can now be used to specify the two connected components of $X_{M, g}$ in the case of a non-compact Cauchy surface. The right component is that containing $(\gamma(0), \gamma(1))$ for the endpoints $\gamma(0)$ and $\gamma(1)$ of some and hence any right-directed spacelike curve $\gamma$. This follows from the previous description in terms of the foliation since the spatial component is strictly increasing along such a curve.

\subsection{Appendix to Chapter 2}

Proof of Lemma 2.1 (Sketch)

Let $\mathcal{O}=\operatorname{int} D(G)$ be a regular diamond, $G \subset C$ where $C$ is an acausal Cauchy-surface, and $p \in \mathcal{O}^{\perp}$.

Choose a $C^{\infty}$-foliation $F: \mathbb{R} \times \Sigma \rightarrow M$ of $M$ into smooth, acausal Cauchy surfaces. Then for each $y \in \Sigma$, the curves $t \mapsto F(t, y)$ are inextendible, futuredirected timelike curves. Therefore, given any acausal Cauchy surface $C_{0}$, each of these curves intersects $C_{0}$ exactly once, at the parameter value $t=\tau_{C_{0}}(y)$. The function $\tau_{C_{0}}: \Sigma \rightarrow \mathbb{R}$ is a smooth function and one has $C_{0}=\left\{F\left(\tau_{C_{0}}(y), y\right): y \in \Sigma\right\}$. Furthermore, the map $\Phi_{C, C_{0}}: C \rightarrow C_{0}$ induced by $F\left(\tau_{C}(y), y\right) \mapsto F\left(\tau_{C_{0}}(y), y\right)$ is a diffeomorphism.

Using the results of $[11$, one can show that there is an open neighbourhood $U$ of $\bar{G}$ in $C$ possessing the same properties (i) and (ii) as $G$, i.e. $U$ is the base of a regular diamond. It is also not difficult to show (cf. [60]) that there exists an acausal Cauchy surface $C_{0}$ containing $p$ and with the additional property that

$$
J(\bar{G}) \cap C_{0} \subset \Phi_{C, C_{0}}(U)=: U_{0} .
$$

The latter property means there are acausal Cauchy surfaces $C_{0}$ passing through $p$ and coming arbitrarily close to $\bar{G}$. This entails that $\mathcal{O}_{0}:=\operatorname{int} D\left(U_{0}\right)$ contains $\mathcal{O}$. Since $\Phi_{C, C_{0}}$ is a diffeomorphism, $U_{0}$ satisfies (i) and (ii) with respect to the Cauchy surface $C_{0}$.

It remains to show that $U_{0} \cup\{p\}$ is contained in a subset $U_{1}$ of $C_{0}$ satisfying (i) and (ii) with respect to the Cauchy surface $C_{0}$. This is done by connecting a point in a smooth part of $\partial U_{0}$ by a smooth curve $\lambda$ to $p$ and by attaching to $U_{0}$ a suitable smooth deformation of a tubular normal neighbourhood of $\lambda$. This yields the required set $U_{1}$; properties (i) and (ii) follow by construction as does

$$
\mathcal{O} \cup\{p\} \subset \operatorname{int} D\left(U_{1}\right)=: \mathcal{O}_{1} .
$$




\section{Superselection Structure in Curved Spacetimes}

\subsection{Introduction}

In this section, we adapt the basic notions and results of the theory of superselection sectors to curved spacetime, limiting ourselves to globally hyperbolic spacetimes. As we shall see, the basic theory goes through smoothly in the case of globally hyperbolic spacetimes with a noncompact Cauchy surface and much of it in the case of a compact Cauchy surface. The geometry of spacetime fortunately enters the long analysis only in establishing a few specific points. We can therefore limit ourselves to clarifying these points and otherwise just quoting the consequences.

We let $\mathcal{K}$ denote the set of regular diamonds in $M$, ordered under inclusion. If $M$ is globally hyperbolic with a non-compact Cauchy surface, $\mathcal{K}$ may not be directed although it will be in cases of interest. However, when $M$ is globally hyperbolic with a compact Cauchy surface, $\mathcal{K}$ will never be directed and we shall meet problems akin to those on the circle. The more complicated structures involved have been relegated, as far as possible, to the appendix to this chapter.

The set of double cones in $M$ whose causal complement has non-empty interior is even less likely to be directed. Both sets have in common that they form a base for the topology of $M$ and we will consider our nets of observables as being defined over $\mathcal{K}$ with the general philosophy that they can be extended to other regions, if necessary. In fact, we will consider a wider class of regions in subsequent chapters. Now, the geometry of spacetime enters the analysis only through the partially ordered set $\mathcal{K}$ and its relation of causal disjointness, introduced below. In view of further applications and despite the degree of abstraction involved, we have emphasised the relevant properties of $\mathcal{K}$.

The selection criterion for localized charges in Minkowski space uses the vacuum representation as a reference. Although there is no such preferred representation in curved spacetime, one expects there to be a preferred collection of representations satisfying the conditions listed in Sec. 1.1. In the case of the Klein-Gordon field on a four dimensional globally hyperbolic spacetime, we may take the representations induced by the pure quasifree Hadamard states [59]. We shall choose one of these representations as our reference representation and, whilst our sectors will depend on this choice, the superselection structure will not since this depends only the net of von Neumann algebras. By 4) of Sec. 1.1, any two preferred representations generate the same net of von Neumann algebras. We will denote our reference representation by $\pi^{0}$ and its Hilbert space by $\mathcal{H}_{0}$.

Once the reference representation has been fixed, it is just the causal structure of Minkowski space that plays a role in the superselection criterion for localized charges. For this reason, it adapts well to curved spacetime. The causal structure enters in the form of the relation $\perp$ of causal disjointness, defined in Ch. 2, and here to be considered as a relation on the ordered set $\mathcal{K}$, satisfying

a) $\mathcal{O}_{1} \perp \mathcal{O}_{2} \Rightarrow \mathcal{O}_{2} \perp \mathcal{O}_{1}$.

b) $\mathcal{O}_{1} \subset \mathcal{O}_{2}$ and $\mathcal{O}_{2} \perp \mathcal{O}_{3} \Rightarrow \mathcal{O}_{1} \perp \mathcal{O}_{3}$. 
c) Given $\mathcal{O}_{1} \in \mathcal{K}$, there exists an $\mathcal{O}_{2} \in \mathcal{K}$ such that $\mathcal{O}_{1} \perp \mathcal{O}_{2}$.

We write $\mathcal{O}^{\perp}:=\left\{\mathcal{O}_{1} \in \mathcal{K}: \mathcal{O}_{1} \perp \mathcal{O}\right\}$.

As explained above, the geometry of spacetime enters through the partially ordered set $\mathcal{K}$ together with the relation $\perp$ of causal disjointness. Hence we have to pass from geometric or topological properties of $(M, g)$ to properties of $(\mathcal{K}, \perp)$. We will need to know whether certain partially ordered sets are connected, a notion defined in the appendix. But the basic idea is to move from one element $\mathcal{O}_{1}$ of $\mathcal{K}$ to a nearby element $\mathcal{O}_{2}$, where nearby means that there is a third element $\mathcal{O}_{3}$ containing $\mathcal{O}_{1}$ and $\mathcal{O}_{2}$. A finite series of such moves constitutes a path. $\mathcal{K}$ is connected if any two elements can be connected by a path. By virtue of Lemma 3A.1, we know that $\mathcal{K}$ is connected and, see Lemma 2.2, that $\mathcal{O}^{\perp}$ is connected except when $M$ is two dimensional with a non-compact Cauchy surface.

Lemma 2.2, itself, asserts that the set $X_{M, g}$ of pairs of spacelike separated points is pathwise connected again unless $M$ is two dimensional with a non-compact Cauchy surface. Since pairs of elements of $\mathcal{K}$ form a base for the topology in the product space, we can again conclude by Lemma 3A.1 that the graph $\mathcal{G}^{\perp}$ of the relation $\perp$ is connected,

$$
\mathcal{G}^{\perp}=\left\{\mathcal{O}_{1} \times \mathcal{O}_{2}: \mathcal{O}_{1} \perp \mathcal{O}_{2}\right\}
$$

In the exceptional case, $X_{M, g}$ has two pathwise connected components. Indeed the causal complement of a point is no longer connected but decomposes into a 'left' causal complement and a 'right' causal complement.

These are the basic geometric considerations determining the statistics. The remaining condition used in Sec. 3.3, the surjectivity of the projection from $\mathcal{G}_{c}^{\perp}$, a connected component of $\mathcal{G}^{\perp}$, to $\mathcal{K}$ has no geometric relevance seeing that it is automatically satisfied in the context of globally hyperbolic spacetimes. Thus, as will follow from the results of Sec. 3.3, in a globally hyperbolic spacetime of dimension greater than 2 , we get a net of symmetric tensor $W^{*}$-categories, $\left(\mathcal{T}_{t}, \varepsilon^{c}\right)$, whereas in a 2-dimensional spacetime we shall in general get a braided tensor $W^{*}$-category with two different braidings $\varepsilon^{\ell}$ and $\varepsilon^{r}$ corresponding to the left and right causal complements of a double cone. Obviously, $\varepsilon^{\ell}=\varepsilon^{r *}$, where $\varepsilon^{*}$ is defined by

$$
\varepsilon^{*}(\rho, \sigma)=\varepsilon(\sigma, \rho)^{*} .
$$

The next basic step is to establish the properties of charge conjugation. The basic tool here is a left inverse. The physical idea behind constructing left inverses is that of transferring charge to spacelike infinity and a geometric property is obviously involved. Expressed as a property of our partially ordered set $\mathcal{K}$ we need to assume the existence of a net $\mathcal{O}_{n}$ of elements of $\mathcal{K}$ such that given $\mathcal{O} \in \mathcal{K}$ there exists an $n_{0}$ with $\mathcal{O}_{n} \perp \mathcal{O}$ for $n \geq n_{0}$. We will say that a net $\mathcal{O}_{n}$ tends spacelike to infinity. Such a net obviously exists whenever $\mathcal{K}$ is directed but it continues to exist for an arbitrary globally hyperbolic spacetime with a noncompact Cauchy surface. The question of whether one can find a suitable substitute for globally hyperbolic spacetimes with compact Cauchy surfaces is still open, a defect mitigated by the circumstance that 
a left inverse exists as a consequence of the equality of local and global intertwiners, postulated in Ch. 5 .

In this way, we establish in Sec. 3.4, the classification of statistics and the existence of charge conjugation for finite statistics for the case of a globally hyperbolic spacetime of dimension greater than two.

\subsection{The Selection Criterion}

Our discussion of superselection theory in this and in subsequent sections is in terms of a partially ordered set $\mathcal{K}$ together with a binary relation $\perp$. The necessary properties will be introduced as needed and there will be no specific reference to spacetime. We have adopted this procedure for clarity and with future applications in mind. Thus the best choice of $\mathcal{K}$ in a curved spacetime is not altogether clear. We have already, for example, thought fit to use regular diamonds in place of double cones. On the other hand, we might like to go beyond strictly localized charges and work with spacelike cones or to replace causal disjointness by its Euclidean counterpart, disjointness, as when working on the circle. In fact, we shall need to use results on superselection structure on the circle in Ch. 4 and, although these results have been developed previously [28], [32], the formalism presented here includes this case and allows a uniform approach to all such problems. We shall also simplify the exposition by making use of the freedom to modify the binary relation on $\mathcal{K}$. Thus this degree of abstraction is now called for even if we have not been able to derive all results in an adequate generality.

Two nets $\mathcal{A}$ and $\mathcal{B}$ of ${ }^{*}$-subalgebras of $\mathcal{B}\left(\mathcal{H}_{0}\right)$ over $\mathcal{K}$ are said to be relatively local if

$$
\mathcal{A}\left(\mathcal{O}_{1}\right) \subset \mathcal{B}\left(\mathcal{O}_{2}\right)^{\prime} \text {, whenever } \mathcal{O}_{1} \perp \mathcal{O}_{2} .
$$

This relation fulfills the analogues of a), b) and c) above. Furthermore, there is a maximal net, the dual net $\mathcal{A}^{d}$, which is relatively local to $\mathcal{A}$. It is given by

$$
\mathcal{A}^{d}(\mathcal{O})=\cap\left\{\mathcal{A}\left(\mathcal{O}_{1}\right)^{\prime}: \mathcal{O}_{1} \perp \mathcal{O}\right\}
$$

Since $\mathcal{A}^{d d}$ is the largest net local relative to $\mathcal{A}^{d}, \mathcal{A} \subset \mathcal{A}^{d d}$. However $\mathcal{A} \subset \mathcal{B}$ implies $\mathcal{B}^{d} \subset \mathcal{A}^{d}$, so that $\mathcal{A}^{d}=\mathcal{A}^{d d d}$. A net $\mathcal{A}$ is said to be local if $\mathcal{A} \subset \mathcal{A}^{d}$ and then $\mathcal{A}^{d d} \subset \mathcal{A}^{d}=\mathcal{A}^{d d d}$ so that $\mathcal{A}^{d d}$ is local, too. We now compute the double dual:

$$
\mathcal{A}^{d d}(\mathcal{O})=\bigcap_{\mathcal{O}_{1} \perp \mathcal{O}} \mathcal{A}^{d}\left(\mathcal{O}_{1}\right)^{\prime}=\bigcap_{\mathcal{O} \perp \mathcal{O}_{1}} \vee_{\hat{\mathcal{O}} \perp \mathcal{O}_{1}} \mathcal{A}(\hat{\mathcal{O}})
$$

Definition. A representation $\pi$ of the net $\mathcal{A}$ is said to satisfy the selection criterion if

$$
\pi \uparrow \mathcal{O}^{\perp} \simeq \pi^{0} \uparrow \mathcal{O}^{\perp}, \quad \mathcal{O} \in \mathcal{K} .
$$

\footnotetext{
${ }^{5}$ Baumgärtel and Wollenberg[2] treat nets over partially ordered sets with a relation of causal disjointness. In their applications to superselection structure they assume among other properties that the partially ordered set is directed. When the partially ordered set is not directed, their notion of representation depends on a choice of enveloping quasilocal algebra.
} 
When $\mathcal{K}$ is directed this means that for each $\mathcal{O}$ there is a unitary $V_{\mathcal{O}}$ such that

$$
V_{\mathcal{O}} \pi(A)=A V_{\mathcal{O}}, \quad A \in \mathcal{A}\left(\mathcal{O}_{1}\right), \quad \mathcal{O}_{1} \in \mathcal{O}^{\perp},
$$

where, to simplify notation in the sequel, we have omitted the symbol $\pi^{0}$ for the reference representation. We write $T \in\left(\pi, \pi^{\prime}\right)$ to mean that $T$ intertwines the representations $\pi$ and $\pi^{\prime}$ and let $\operatorname{Rep}^{\perp} \mathcal{A}$ denote the $W^{*}$-category whose objects are the representations of $\mathcal{A}$ satisfying the selection criterion and whose arrows are the intertwiners between these representations. As far as superselection theory goes, the following result allows one to replace the original net by its bidual.

The Extension Theorem If each $\mathcal{O}^{\perp}$ is connected, every object $\pi$ of $\operatorname{Rep}^{\perp} \mathcal{A}$ admits a unique extension to an object of $\operatorname{Rep}^{\perp} \mathcal{A}^{d d}$. Furthermore there is a canonical isomorphism of the corresponding $W^{*}$-categories.

This result is proved as Theorem 3A.4 of the Appendix. How to proceed when $\mathcal{O}^{\perp}$ is not connected is exemplified by the well known case of a two dimensional Minkowski space and we will not attempt a general analysis here. The theory of superselection structure rests on two assumptions. The first is a property derived by Borchers in Minkowski space as a consequence of additivity, locality and the spectrum condition. Here it involves the dual net, $\mathcal{A}^{d}$.

Definition A net $\mathcal{A}^{d}$ satisfies Property $B$ if given $\mathcal{O}, \mathcal{O}_{1}$ and $\mathcal{O}_{2}$ in $\mathcal{K}$ such that $\mathcal{O} \perp \mathcal{O}_{2}$, and $\mathcal{O}, \mathcal{O}_{2} \subset \mathcal{O}_{1}$ and a projection $E \neq 0$ in $\mathcal{A}^{d}(\mathcal{O})$, there is an isometry $W \in \mathcal{A}^{d}\left(\mathcal{O}_{1}\right)$ with $W W^{*}=E$.

Lemma 3.1 If $\mathcal{A}^{d}$ satisfies Property $B$, the set of representations satisfying the selection criterion is closed under direct sums and (non-trivial) subrepresentations. In other words, the $W^{*}$-category $\operatorname{Rep}^{\perp} \mathcal{A}$ has direct sums and (non-zero) subobjects.

The proof of this lemma will be omitted as it in no way differs from its Minkowski counterpart. The characteristic assumption of superselection theory is a duality assumption.

Definition A net $\mathcal{A}$ is said to satisfy duality if $\mathcal{A}=\mathcal{A}^{d}$ and essential duality if $\mathcal{A}^{d d}=\mathcal{A}^{d}$.

To simplify notation, we shall suppose here that our net satisfies duality but, as a consequence of the Extension Theorem, the results remain valid under the weaker assumption of essential duality, whenever each $\mathcal{O}^{\perp}$ is connected.

In the Appendix, we have adopted the cohomological approach to superselection structure as this provides the most natural expression of the selection criterion. In the main text, we shall pursue the alternative strategy of working in terms of localized endomorphisms rather than 1-cocycles.

\subsection{Localized Endomorphisms}

When $\mathcal{K}$ is directed, the analysis of superselection structure rests on the following simple construction: let $\pi$ be a representation satisfying the selection criterion, pick a unitary $V_{\mathcal{O}}$ as above and set

$$
\rho(A):=V_{\mathcal{O}} \pi(A) V_{\mathcal{O}}^{*}, \quad A \in \mathcal{A} .
$$


Obviously $\rho$ is a representation of $\mathcal{A}$ on $\mathcal{H}_{0}$ unitarily equivalent to $\pi$ but, in fact, $\rho(\mathcal{A}) \subset \mathcal{A}$. To see this, pick $\mathcal{O}_{1}, \mathcal{O}_{2} \in \mathcal{K}, \mathcal{O}_{1} \supset \mathcal{O}, \mathcal{O}_{1} \perp \mathcal{O}_{2}$ and $B \in \mathcal{A}\left(\mathcal{O}_{2}\right)$ then, writing $V$ for $V_{\mathcal{O}}$,

$$
\rho(A) B=V \pi(A) V^{*} B=V \pi(A B) V^{*}=V \pi(B A) V^{*}=B V \pi(A) V^{*}=B \rho(A),
$$

Hence $\rho(A) \in \mathcal{A}^{d}\left(\mathcal{O}_{1}\right)=\mathcal{A}\left(\mathcal{O}_{1}\right)$, as required. Furthermore, $\rho$ is localized in $\mathcal{O}$, i.e.

$$
\rho(A B)=\rho(A) B, \quad B \in \mathcal{A}\left(\mathcal{O}_{1}\right), \quad A \in \mathcal{A}, \quad \mathcal{O}_{1} \perp \mathcal{O}
$$

and we refer to $\rho$ as a localized endomorphism. Now if $\rho$ and $\rho^{\prime}$ are localized endomorphisms, an intertwiner $R$ for the corresponding representations is automatically in $\mathcal{A}$. For suppose $\rho$ and $\rho^{\prime}$ are localized in $\mathcal{O}$ and $A \in \mathcal{A}\left(\mathcal{O}_{1}\right), \mathcal{O}_{1} \perp \mathcal{O}$, then

$$
R A=R \rho(A)=\rho^{\prime}(A) R=A R
$$

so that $R \in \mathcal{A}^{d}(\mathcal{O})=\mathcal{A}(\mathcal{O})$.

We can thus write $R \in\left(\rho, \rho^{\prime}\right)$ without specifying whether we treat $\rho$ as a representation or as an endomorphism and, when studying superselection sectors, $\operatorname{Rep}^{\perp} \mathcal{A}$ may be replaced by the full subcategory $\mathcal{T}_{t}$ of $\operatorname{End} \mathcal{A}$. End $\mathcal{A}$ is a tensor $C^{*}$-category and we use the tensor product notation. Thus if $S \in\left(\sigma, \sigma^{\prime}\right)$, we write $R \otimes S$ to denote the intertwiner $R \rho(S) \in\left(\rho \sigma, \rho^{\prime} \sigma^{\prime}\right)$. We characterize $\mathcal{T}_{t}$ by characterizing the corresponding set $\Delta_{t}$ of endomorphisms. The representation corresponding to $\rho \in \Delta_{t}$ satisfies the selection criterion precisely when, given $\mathcal{O} \in \mathcal{K}$, there is an equivalent endomorphism $\sigma$ localized in $\mathcal{O}$. We then call $\rho$ transportable since, transporting $\rho$ by a suitable unitary $U \in \mathcal{A}$, it can be localized in any given $\mathcal{O} \in \mathcal{K} . \Delta_{t}$ is thus the set of transportable localized endomorphisms and $\Delta_{t}(\mathcal{O})$ shall denote the subset of endomorphisms localized in $\mathcal{O}$.

Lemma 3.2 If $\rho, \rho^{\prime} \in \Delta_{t}$ then $\rho \rho^{\prime} \in \Delta_{t}$.

Proof. As the product of endomorphisms localized in $\mathcal{O}$ is again localized in $\mathcal{O}$, it suffices to observe that if $U \in(\rho, \sigma)$ and $U^{\prime} \in\left(\rho^{\prime}, \sigma^{\prime}\right)$ are unitary then $U \otimes U^{\prime} \in$ $\left(\rho \rho^{\prime}, \sigma \sigma^{\prime}\right)$ is unitary.

Thus the unitary equivalence class of $\rho \rho^{\prime}$ depends only on the unitary equivalence classes of $\rho$ and $\rho^{\prime}$ and, regarding charge as the quality distinguishing one sector from another, this defines a composition of charges.

When $\mathcal{K}$ is not directed, this simple scheme must be modified. The basic complication is that localized endomorphisms are now not defined on the whole net $\mathcal{A}$. Instead, an endomorphism $\rho$ localized in $\mathcal{O}$ is just defined on the net $\mathcal{O}_{1} \mapsto \mathcal{A}\left(\mathcal{O}_{1}\right)$ with $\mathcal{O} \subset \mathcal{O}_{1}$ and has the property that $\rho\left(\mathcal{A}\left(\mathcal{O}_{1}\right)\right) \subset \mathcal{A}\left(\mathcal{O}_{1}\right)$. As explained in detail in the Appendix, we have a net $\mathcal{O} \mapsto \mathcal{T}_{t}(\mathcal{O})$ of tensor $W^{*}$-categories, the objects of $\mathcal{T}_{t}(\mathcal{O})$ are the transportable endomorphisms localized in $\mathcal{O}$.

It is also shown in the Appendix how a representation $\pi$ satisfying the selection criterion gives rise to objects of $\mathcal{T}_{t}(a), a \in \Sigma_{0}$ and how an interwiner $T \in\left(\pi, \pi^{\prime}\right)$ between two such representations leads to arrows $t_{a}, a \in \Sigma_{0}$, between the corresponding 
objects of $\mathcal{T}_{t}(a)$. We can no longer study superselection sectors replacing $\operatorname{Rep} \mathcal{A}^{\perp}$ by $\mathcal{T}_{t}(\mathcal{O})$, more precisely, we have a faithful ${ }^{*}$-functor from $\operatorname{Rep} \mathcal{A}^{\perp}$ to $\mathcal{T}_{t}(\mathcal{O})$ but cannot assert that it is an equivalence of $W^{*}$-categories. Thus, when $\mathcal{K}$ is not directed, $\mathcal{T}_{t}(\mathcal{O})$ may not give a description of superselection sectors. Nevertheless, as we shall see, an analysis of localized endomorphisms still provides useful information.

The basic step in this analysis is to investigate the relation between causal disjointness and commutation of localized endomorphisms and their intertwiners. It is natural to say that an intertwiner $T \in \mathcal{T}_{t}(\mathcal{O})$ is localized in $\mathcal{O}$, but we need a finer notion because we may have $T \in\left(\rho_{1}, \rho_{0}\right)$ where $\rho_{i} \in \Delta_{t}\left(\mathcal{O}_{i}\right)$ with $\mathcal{O}_{i} \subset \mathcal{O}$. In this case, we refer to $\mathcal{O}_{1}$ as being an initial support and $\mathcal{O}_{0}$ as being a final support of $T$. As explained in the Appendix, we consider the set $\Sigma_{1}$ of 1 -simplices in $\mathcal{K}$ as a partially ordered set and let $\Sigma_{1}^{\perp}$ denote the subset of 1 -simplices $b$ with $\partial_{1} b \perp \partial_{0} b$ with the induced order.

Lemma 3.3 Let $\Sigma_{1, c}^{\perp}$ be a connected component of $\Sigma_{1}^{\perp}$, and suppose that given $\mathcal{O}_{0} \in \mathcal{K}$, there is a $b \in \Sigma_{1, c}^{\perp}$ with $\partial_{0} b=\mathcal{O}_{0}$. Let $T_{i} \in\left(\rho_{i}, \rho_{i}^{\prime}\right)$ be arrows in some $\mathcal{T}_{t}(\mathcal{O})$ then

$$
T_{0} \otimes T_{1}=T_{1} \otimes T_{0}
$$

if there are $b, b^{\prime} \in \Sigma_{1, c}^{\perp}$ so that $\partial_{0} b$ and $\partial_{1} b$ are initial supports of $T_{0}$ and $T_{1}$ and $\partial_{0} b^{\prime}$ and $\partial_{1} b^{\prime}$ are final supports of $T_{0}$ and $T_{1}$.

Proof. We first show that $T_{0} \rho_{0}\left(T_{1}\right)=T_{1} \rho_{1}\left(T_{0}\right)$. This relation is trivial if $T_{0}$ and $T_{1}$ are causally disjoint in the sense that there is a $\hat{b} \in \Sigma_{1}^{\perp}$ such that $\partial_{0} \hat{b}$ contains an initial and final support of $T_{0}$ and $\partial_{1} \hat{b}$ an initial and final support of $T_{1}$. The idea of the proof is to reduce to this trivial case. Replace $T_{0}$ and $T_{1}$ by $T_{2}=T_{0} \circ U_{0}$ and $T_{3}=T_{1} \circ U_{1}$, where $U_{0} \in\left(\rho_{2}, \rho_{0}\right)$ and $U_{1} \in\left(\rho_{3}, \rho_{1}\right)$ are unitary. Then

$$
T_{2} \otimes T_{3}=T_{0} \otimes T_{1} \circ U_{0} \otimes U_{1}, \quad T_{3} \otimes T_{2}=T_{1} \otimes T_{0} \circ U_{1} \otimes U_{0},
$$

to be understood as valid in some $\mathcal{T}_{t}(\hat{\mathcal{O}})$ for $\hat{\mathcal{O}}$ sufficiently large. Thus if $U_{0}$ and $U_{1}$ are causally disjoint, the validity or not of our relation is unaffected by the passage from $T_{0}, T_{1}$ to $T_{2}, T_{3}$. But $b$ and $b^{\prime}$ lie in a connected component $\Sigma_{1, c}^{\perp}$ by hypothesis, so after a finite number of steps we can arrange that the initial and final supports of both intertwiners coincide. This is again the trivial case so $T_{0} \rho_{0}\left(T_{1}\right)=T_{1} \rho_{1}\left(T_{0}\right)$, as required. It only remains to show that

$$
\rho_{0} \rho_{1}-\rho_{1} \rho_{0}=0
$$

The above computations show that the kernel of the left hand side does not change if we shift to $\rho_{2}$ and $\rho_{3}$. However, by hypothesis, given $\mathcal{O} \supset b^{\prime}$, we can find $\hat{b} \in \Sigma_{1, c}^{\perp}$ with $\partial_{0} \hat{b}=\mathcal{O}$ and we can take $\rho_{3} \in \Delta_{t}\left(\partial_{1} \hat{b}\right)$, when

$$
\rho_{0} \rho_{3}(A)=\rho_{0}(A)=\rho_{3} \rho_{0}(A), \quad A \in \mathcal{A}(\mathcal{O}),
$$

completing the proof. 
After this one crucial lemma, the standard results on the existence of a braiding follow without further geometric input. Of course the braiding will, in general, continue to depend on the choice of connected component.

Theorem 3.4 Let $\Sigma_{1, c}^{\perp}$ be a connected component of $\Sigma_{1}^{\perp}$. If the projection mapping $b \mapsto \partial_{0} b$ from $\Sigma_{1, c}^{\perp}$ to $\mathcal{K}$ is surjective then there is a unique intertwiner-valued function $\left(\rho_{0}, \rho_{1}\right) \mapsto \varepsilon^{c}\left(\rho_{0}, \rho_{1}\right) \in\left(\rho_{0} \rho_{1}, \rho_{1} \rho_{0}\right)$ such that

a) $\varepsilon^{c}\left(\rho_{0}^{\prime}, \rho_{1}^{\prime}\right) \circ T_{1} \otimes T_{2}=T_{2} \otimes T_{1} \circ \varepsilon^{c}\left(\rho_{0}, \rho_{1}\right), \quad T_{i} \in\left(\rho_{i}, \rho_{i}^{\prime}\right), \quad i=0,1$,

b) $\varepsilon^{c}\left(\rho_{0}, \rho_{1}\right)=1_{\rho_{0} \rho_{1}}$, if there is a $b \in \Sigma_{1, c}^{\perp}$ such that $\rho_{i} \in \Delta_{t}\left(\partial_{i} b\right) i=0,1$.

Proof. The uniqueness claim tells us how to go about defining $\varepsilon^{c}$ : given $\rho_{1}, \rho_{2}$ pick $b \in \Sigma_{1, c}^{\perp}$ and unitaries $U_{i} \in\left(\rho_{i}, \tau_{i}\right)$ where $\tau_{i} \in \Delta_{t}\left(\partial_{i} b\right)$ and we have no option but to set

$$
\varepsilon^{c}\left(\rho_{1}, \rho_{2}\right)=U_{2}^{*} \otimes U_{1}^{*} \circ U_{1} \otimes U_{2} .
$$

By Lemma 3.3, such a choice, however made, automatically satisfies $b$ ). We have $\varepsilon^{c}\left(\rho_{1}^{\prime}, \rho_{2}^{\prime}\right)=U_{2}^{\prime *} \otimes U_{1}^{\prime *} \circ U_{1}^{\prime} \otimes U_{2}^{\prime}$, where $U_{i}^{\prime} \in\left(\rho_{i}^{\prime}, \tau_{i}^{\prime}\right)$ and the product of supports of $\tau_{1}^{\prime}$ and $\tau_{2}^{\prime}$ is contained in $X_{c}$. Set $S_{i}=U_{i}^{\prime} \circ T_{i} \circ U_{i}^{*}$ then, by Lemma 3.3, $S_{1} \otimes S_{2}=S_{2} \otimes S_{1}$ and rearranging this identity gives $a$ ) and completes the proof of the theorem.

Corollary 3.5 Under the hypothesis of Theorem 3.4

a) $\varepsilon^{c}\left(\rho_{1} \rho_{2}, \rho_{3}\right)=\varepsilon^{c}\left(\rho_{1}, \rho_{3}\right) \otimes 1_{\rho_{2}} \circ 1_{\rho_{1}} \otimes \varepsilon^{c}\left(\rho_{2}, \rho_{3}\right)$,

b) $\varepsilon^{c}\left(\rho_{1}, \rho_{2} \rho_{3}\right)=1_{\rho_{2}} \otimes \varepsilon^{c}\left(\rho_{1}, \rho_{3}\right) \circ \varepsilon^{c}\left(\rho_{1}, \rho_{2}\right) \otimes 1_{\rho_{3}}$,

If $b \in \Sigma_{1, c}^{\perp}$ implies $\bar{b} \in \Sigma_{1, c}^{\perp}$, where $|\bar{b}|=|b|, \partial_{0} \bar{b}=\partial_{1} b$ and $\partial_{1} \bar{b}=\partial_{0} b$, then

c) $\varepsilon^{c}\left(\rho_{2}, \rho_{1}\right) \circ \varepsilon^{c}\left(\rho_{1}, \rho_{2}\right)=1_{\rho_{1} \rho_{2}}$.

Proof. These equalities follow easily from the formula

$$
\varepsilon^{c}\left(\rho_{1}, \rho_{2}\right)=U_{2}^{*} \otimes U_{1}^{*} \circ U_{1} \otimes U_{2}
$$

used to define $\varepsilon^{c}$ in the proof of Theorem 3.4.

As a consequence of $a$ ) and $b$ ) or by direct computation, we also have

$$
\varepsilon^{c}(\rho, \iota)=\varepsilon^{c}(\iota, \rho)=1_{\rho} .
$$

In virtue of $a$ ) and $b$ ), if $\mathcal{K}$ is directed, the pair $\left(\mathcal{T}_{t}, \varepsilon^{c}\right)$ is a braided tensor $W^{*}$-category and when $c$ ) holds, too, we get a symmetric tensor $W^{*}$-category. In the general case we get a net $\mathcal{O} \mapsto\left(\mathcal{T}_{t}(\mathcal{O}), \varepsilon^{c}\right)$ of braided or symmetric tensor $W^{*}$-categories, where the terminology implies that the inclusion $\mathcal{T}_{t}\left(\mathcal{O}_{1}\right) \subset \mathcal{T}_{t}\left(\mathcal{O}_{2}\right)$ for $\mathcal{O}_{1} \subset \mathcal{O}_{2}$ is not only a tensor ${ }^{*}$-functor but also preserves the braiding. 
In view of the above results, it is obviously important to be able to compute the connected components of $\Sigma_{1}^{\perp}$. We first localize and try to compute the connected components of

$$
\Sigma_{1}^{\perp}(\mathcal{O}):=\left\{b \in \Sigma_{1}^{\perp}:|b| \subset \mathcal{O}\right\}
$$

before trying to compute those of $\Sigma_{1}^{\perp}$. Needleess to say, neither step can be carried through at this level of generality but we shall carry them through when $\mathcal{K}$ is the set of regular diamonds in a globally hyperbolic spacetime.

Note that $\Sigma_{1}^{\perp}(\mathcal{O})$ is closely related to the local graph of the relation $\perp$,

$$
\mathcal{G}^{\perp}(\mathcal{O}):=\left\{\mathcal{O}_{1} \times \mathcal{O}_{0}: \mathcal{O}_{1}, \mathcal{O}_{0} \subset \mathcal{O}, \mathcal{O}_{1} \perp \mathcal{O}_{0}\right\}
$$

There is an obvious order-preserving injection $i: \mathcal{G}^{\perp}(\mathcal{O}) \rightarrow \Sigma^{\perp}(\mathcal{O})$. We simply consider $\mathcal{O}_{i}$ as $\partial_{i} b$ and $\mathcal{O}$ as $|b|$. Conversely, we have an order-preserving surjection $s: \Sigma_{1}^{\perp}(\mathcal{O}) \rightarrow \mathcal{G}^{\perp}(\mathcal{O})$ mapping $b$ to $\partial_{1} b \times \partial_{0} b . \quad b$ lies in the same component of $\Sigma_{1}^{\perp}(\mathcal{O})$ as $i \circ s(b)$. Hence if $s(b)$ and $s\left(b^{\prime}\right)$ lie in the same component, so do $b$ and $b^{\prime}$, thus we have computed the components of $\Sigma_{1}^{\perp}(\mathcal{O})$ in terms of those of $\mathcal{G}^{\perp}(\mathcal{O})$. Now if $\mathcal{O}$ is a regular diamond in a globally hyperbolic spacetime, then $\mathcal{O}$ itself with the induced metric is a globally hyperbolic spacetime with a non-compact Cauchy surface and the connected components have been computed in Lemma 2.2.

For passing from the local to the global computation, the strategy is to look for coherent choices of components for the $\Sigma_{1}^{\perp}(\mathcal{O})$, i.e. we want a component $\Sigma_{1, c}^{\perp}(\mathcal{O})$ for each $\mathcal{O}$ such that

$$
\Sigma_{1, c}^{\perp}\left(\mathcal{O}_{1}\right)=\Sigma_{1, c}^{\perp}\left(\mathcal{O}_{2}\right) \cap \Sigma_{1}^{\perp}\left(\mathcal{O}_{1}\right), \quad \mathcal{O}_{1} \subset \mathcal{O}_{2}
$$

Lemma 3.6 Given a coherent choice of components $\mathcal{O} \mapsto \Sigma_{1, c}^{\perp}(\mathcal{O})$, then $\Sigma_{1, c}^{\perp}:=\{b \in$ $\left.\Sigma_{1}: b \in \Sigma_{1, c}^{\perp}(|b|)\right\}$ is a component of $\Sigma_{1}^{\perp}$.

Proof. $\mathcal{K}$ being connected, the result will follow from Lemma 3A.3 once we show that

$$
\Sigma_{1, c}^{\perp}(\mathcal{O})=\Sigma_{1, c}^{\perp} \cap \Sigma_{1}^{\perp}(\mathcal{O})
$$

But if $b \in \Sigma_{1, c}^{\perp}(\mathcal{O}),|b| \subset \mathcal{O}$ and since we have a coherent choice of components, $b \in \Sigma_{1, c}^{\perp}(|b|)$ giving an inclusion. The reverse inclusion is trivial, completing the proof.

Now when $\mathcal{K}$ denotes the set of regular diamonds in globally hyperbolic spacetime with dimension $\geq 2$, then $\Sigma_{1}^{\perp}(\mathcal{O})$ has a single component so that $\Sigma_{1}^{\perp}$ is connected by Lemma 3.6. It remains to consider the case of a globally hyperbolic spacetime of dimension two. We know that each $\Sigma_{1}^{\perp}(\mathcal{O})$ now has two components and that one passes from one component to the other by reversing the orientation of the 1 simplices. We need a way of specifying a coherent choice of components. If the Cauchy surfaces are non-compact, then $\mathcal{G}^{\perp}$ also has two components and one passes from one component to the other by interchanging the two double cones. Hence mapping $b$ to $\partial_{1} b \times \partial_{0} b$ must map the two components of $\Sigma_{1}^{\perp}(\mathcal{O})$ into different components of $\mathcal{G}^{\perp}$. Denoting the two components of $\mathcal{G}^{\perp}$ by $\mathcal{G}_{\ell}^{\perp}$ and $\mathcal{G}_{r}^{\perp}$, the inverse images under 
the above map give us a coherent choice of components. Lemma 3.6 then shows us that $\Sigma_{1}^{\perp}$ has precisely two components and that one passes from one component to the other by reversing the orientation of 1 -simplices.

On the other hand, in a globally hyperbolic spacetime $(M, g)$ of dimension two with compact Cauchy surfaces, we know from the discussion in Sec. 3.1 that $\mathcal{G}^{\perp}$ is connected. However, $\Sigma_{1}^{\perp}$ continues to have two components and we need a different procedure for making a coherent choice of local components. To this end, we pick a nowhere vanishing timelike vector field and restricting this to a regular diamond $\mathcal{O}$, we have, by the discussion following Lemma 2.2, a coherent way of distinguishing the left and right components of the set of spacelike points in the regular diamond and hence left and right components of $\mathcal{G}^{\perp}(\mathcal{O})$ and $\Sigma_{1}^{\perp}(\mathcal{O})$. Thus by Lemma 3.6, $\Sigma_{1}^{\perp}$ has two connected components and one passes from one component to the other by reversing the orientation of 1 -simplices.

\subsection{The Left Inverse and Charge Transfer}

The classification of statistics makes essential use of left inverses. When $\mathcal{K}$ is directed, we may proceed as follows.

Definition A positive linear mapping $\phi$ on $\mathcal{B}\left(\mathcal{H}_{0}\right)$ is called a left inverse of a representation $\pi$ of $\mathcal{A}$ on $\mathcal{H}_{0}$ if

$$
\phi(A \pi(B))=\phi(A) B, \quad A \in \mathcal{B}\left(\mathcal{H}_{0}\right), B \in \mathcal{A}, \quad \text { and } \quad \phi(1)=1 .
$$

There are some simple facts to be noted: first, a positive mapping is automatically self-adjoint, $\phi\left(A^{*}\right)=\phi(A)^{*}$ so that we have $\phi(\pi(A) B)=A \phi(B), A, B \in \mathcal{A}$. Secondly, if $\rho(B)=B$, then $\phi(B)=B$. Thus $\phi$ inherits any localization properties of $\pi$. In particular, if $\pi$ is localized in $\mathcal{O}$

$$
\phi(A)=A \quad \text { for } \quad A \in \mathcal{A}\left(\mathcal{O}_{2}\right), \quad \mathcal{O}_{2} \perp \mathcal{O}
$$

and, by duality, if $\mathcal{O} \subset \mathcal{O}_{1}$ then $\phi\left(\mathcal{A}\left(\mathcal{O}_{1}\right)\right) \subset \mathcal{A}\left(\mathcal{O}_{1}\right)$. Consequently $\phi$ maps $\mathcal{A}$ into $\mathcal{A}$. Furthermore one may show that $\phi\left(A^{*} A\right) \geq \phi(A)^{*} \phi(A)$ and $\|\phi\| \leq 1$.

The complications involved when $\mathcal{K}$ is not directed are treated in the Appendix where the relations with the left inverse of a localized endomorphism and the left inverse of a cocycle are also discussed.

Once we have left inverses, we may proceed to the classification of statistics. We suppose we have permutation statistics. The basic result, stated abstractly, is as follows.

Theorem 3.10 Let $\rho$ be an object in a symmetric tensor $C^{*}$-category $(\mathcal{T}, \varepsilon)$ and $\phi$ a left inverse of $\rho$ with $\phi_{\rho, \rho}=\lambda 1_{\rho}$ for some scalar $\lambda$ then $\lambda \in\{0\} \cup\left\{ \pm d^{-1}: d \in \mathbb{N}\right\}$ and depends only on the equivalence class of $\rho$. The Young tableaux associated with the representations of $\mathbb{P}_{n}$ on $\left(\rho^{n}, \rho^{n}\right), n \geq 1$ are all Young tableaux:

a) whose columns have length $\leq d$, if $\lambda=d^{-1}$ (para-Bose statistics of order $d$ );

b) whose rows have length $\leq d$ if $\lambda=-d^{-1}$ (para-Fermi statistics of order $d$ ); 
c) without restriction, if $\lambda=0$ (infinite statistics).

Note that when $\rho$ is irreducible, $\phi_{\rho, \rho}(\varepsilon(\rho, \rho))$ is automatically a scalar, called the statistics parameter of $\rho . d$ is referred to as the statistics dimension and the sign is the statistics phase, $\kappa_{\rho}$ and corresponds to the Bose-Fermi alternative. In general, we say that $\rho$ has infinite statistics if there is a left inverse $\phi$ with $\phi_{\rho, \rho}(\varepsilon(\rho, \rho))=0$. Otherwise $\rho$ is said to have finite statistics. Assuming our category $\mathcal{T}$ has subobjects, $\rho$ has finite statistics if and only if $\rho$ is a finite direct sum of irreducible objects with finite statistics. In the cases where we can have braid statistics there is, of course, no correspondingly complete classification, not even if we invoke the special setting of a two dimensional Minkowski space. However, many partial results are known in that case and the proofs presumably generalize without essential modification.

As explained in Sec. 3.1, to deduce the existence of a left inverse, we assume that $\mathcal{K}$ has an asymptotically causally disjoint net $\mathcal{O}_{n}$. Thus, given $\mathcal{O} \in \mathcal{K}$ there is an $n_{0}$ with $\mathcal{O}_{n} \perp \mathcal{O}$ for $n \geq n_{0}$. Under such a hypothesis, every representation $\pi$ satisfying the selection criterion can be obtained as a limit of unitary transformations. Physically, this would be interpreted as creating charge by transferring it from spacelike infinity. We pick unitary intertwiners $U_{n} \in\left(\pi_{n}, \pi\right)$ where $\pi_{n}$ is localized in $\mathcal{O}_{n}$. The corresponding unitary transformation $\sigma_{U_{n}}, \sigma_{U_{n}}(A):=U_{n} A U_{n}^{*}$, may be interpreted as an operation which transfers charge from $\mathcal{O}_{n}$ to $\mathcal{O}$. Now if $A \in \mathcal{A}\left(\mathcal{O}_{0}\right)$ and $n$ is sufficiently large so that $\mathcal{O}_{0} \perp \mathcal{O}_{n}$ then $\sigma_{U_{n}}(A)=\pi(A)$ so that, as far as $A$ is concerned, we have created a charge in $\mathcal{O}$. In the limit as $n \rightarrow \infty$ this holds for all $A \in \mathcal{A}$ and we have

Lemma $3.11 \lim _{k \rightarrow \infty}\left\|U_{k} A U_{k}^{*}-\pi(A)\right\|=0, A \in \mathcal{A}$.

The physical idea is now to create the conjugate charge in $\mathcal{O}$ by transferring charge to spacelike infinity. More prosaically, we would like to get a left inverse by replacing $U_{k}$ by $U_{k}^{*}$ and taking a limit. This will indeed be the case although the limiting procedure is more delicate and we cannot work in the strong topology (i.e. pointwise norm topology) for linear mappings on $\mathcal{A}$.

We consider the space $\mathcal{M}$ of bounded linear mappings on $\mathcal{B}\left(\mathcal{H}_{0}\right)$ equipped with the pointwise $\sigma$-topology, i.e. a net $\phi_{n}$ from $\mathcal{M}$ converges to $\phi$ if $\phi_{n}(A)$ converges to $\phi(A)$ in the $\sigma$-topology for each $A \in \mathcal{A}$. The important fact for our purposes is that the unit ball $\mathcal{M}_{1}$ of $\mathcal{M}$ is compact in this topology,

$$
\mathcal{M}_{1}=\{\phi \in \mathcal{M}:\|\phi\| \leq 1\} .
$$

Lemma 3.12 The net $\sigma_{U_{n}^{*}}$ possesses at least one limit point in $\mathcal{M}$. Every limit point of this net is a left inverse of $\pi$. The set of all left inverses of $\rho$ is a nonvoid compact convex subset of $\mathcal{M}$.

We omit the proof as it is identical with that already given for Minkowski space. The existence of an asymptotically causally disjoint net $\mathcal{O}_{n}$ is also used in the analysis of left inverses but there are no new geometric properties involved.

Another important aspect of superselection structure which does not involve spacetime symmetries is the existence of a complete field net with gauge symmetry describing the superselection sectors [25]. This clearly involves no further input 
of a geometric nature as it is based on Corollary 6.2 of 24] which refers to a single $C^{*}$-algebra rather than a net of von Neumann algebras. We leave to the reader the task of formulating a precise result so as to avoid having to introduce the relevant definitions from [25].

\subsection{Sectors of a Fixed-Point Net}

Although we have now succeeded in adapting the main results of superselection theory to globally hyperbolic spacetimes with non-compact Cauchy surface, there is another important aspect to be discussed. As we have seen the Selection Criterion has a natural mathematical extension to curved spacetime. In Minkowski space, however, it is further justified by there being a simple mechanism producing examples of such sectors. Under rather general conditions, it suffices to begin with a field net $\mathcal{F}$ in its vacuum representation and a group of unitaries, a gauge group, compact in the strong operator topology, and inducing automorphisms of the field net. Then defining an observable net $\mathcal{A}$ as the fixed-point net: $\mathcal{A}(\mathcal{O}):=\mathcal{F}(\mathcal{O})^{G}$, the resulting representation decomposes as a direct sum of irreducible representations satisfying the selection criterion. The equivalence classes of these representations are in 1-1 correspondence with the set $\hat{G}$ of equivalence classes of irreducible, continuous, unitary representations of $G$ and the irreducible representation corresponding to $\xi \in \hat{G}$ has multiplicity $d(\xi)$, the dimension of $\xi$. The question is whether these results continue to hold in curved spacetime.

The original result in 23 does not, as it stands, apply to curved spacetime as it involves translations and the cluster property. However the variant given in 25 involves only structural elements and geometric properties compatible with curved spacetime and therefore can be stated here as a result on superselection sectors in curved spacetime. In fact, the following result is valid for a directed set $\mathcal{K}$ with a binary relation $\perp$ such that given $\mathcal{O} \in \mathcal{K}$, there exists $\mathcal{O}_{1}, \mathcal{O}_{2} \in \mathcal{K}$ with $\mathcal{O}, \mathcal{O}_{1} \subset \mathcal{O}_{2}$ and $\mathcal{O} \perp \mathcal{O}_{1}$. This condition is related to our use of the Borchers Property.

Theorem 3.13 Let $\mathcal{F}$ be a field net over $\mathcal{K}$ acting irreducibly on a Hilbert space $\mathcal{H}$ equipped with a strongly compact group $G$ of unitaries inducing automorphisms of the net $\mathcal{F}$. We define the observable net $\mathcal{A}$ to be the fixed-point net:

$$
\mathcal{A}(\mathcal{O}):=\mathcal{F}(\mathcal{O})^{G}, \quad \mathcal{O} \in \mathcal{K} .
$$

We assume that the subspace $\mathcal{H}_{0}$ of $G$-invariant vectors is separable and that $\mathcal{A}$ is represented irreducibly on $\mathcal{H}_{0}$, satisfying duality there and having the Borchers Property. Furthermore, $\mathcal{H}_{0}$ is supposed to be cyclic for each $\mathcal{F}(\mathcal{O})$ and $\mathcal{F}\left(\mathcal{O}_{1}\right)$ and $\mathcal{A}\left(\mathcal{O}_{2}\right)$ to commute whenever $\mathcal{O}_{1} \perp \mathcal{O}_{2}$. Then $\mathcal{A}^{\prime}=G^{\prime \prime}$ and letting $\pi$ denote the defining representation of $\mathcal{A}$ on $\mathcal{H}$

$$
\pi=\sum_{\xi} d(\xi) \pi_{\xi}, \quad \xi \in \hat{G}
$$

where the $\pi_{\xi}$ are inequivalent irreducible representations satisfying the selection criterion and $\hat{G}$ denotes the set of equivalence classes of continuous irreducible unitary representations of $G$. 
Despite this positive result, we must examine the assumptions carefully to see whether they remain reasonable in the context of curved spacetime. To test the assumptions we turn to the examples of scalar free fields defined using quasifree Hadamard states 59. It is known that duality holds for the Klein-Gordon field on a globally hyperbolic spacetime for regular diamonds and that the associated von Neumann algebra is the hyperfinite type $\mathrm{III}_{1}$ factor and hence satisfies the Borchers property. However, at least in the context of Theorem 3.12, this must be regarded as a field net rather than an observable net. Furthermore, we actually use duality for the modified relation $\tilde{\perp}$ of causal disjointness to pass from cocycles to localized endomorphisms in the next section. This strengthened form of duality is equivalent to the original form whenever the nets are inner regular, as is the case for the KleinGordon field. An even stronger form of duality, $\hat{\perp}$-duality, is used in the discussion of left inverses in the next section. However, our basic result on regular diamonds, Lemma 2.1, shows that it is in fact equivalent to $\perp$-duality for additive nets.

As is well known, a geometric property is involved in passing from duality for the fields to duality for the observables. We give here a variant on the proof of Theorem 4.3 of [52], not a priori requiring each irreducible representation of the gauge group to be realized on Hilbert spaces in $\mathcal{F}$. In view of the $\mathbb{Z}_{2}$-graded structure of a field net, it is appropriate to define its dual net by

$$
\mathcal{F}^{d}(\mathcal{O})=\cap\left\{\mathcal{F}^{t}\left(\mathcal{O}_{1}\right)^{\prime}: \mathcal{O}_{1} \perp \mathcal{O}\right\} .
$$

Here $\mathcal{F}^{t}$, the twisted field net, can be defined as the transform of $\mathcal{F}$ under the unitary transformation $2^{-1 / 2}(1+i V)$, where $V$ is the gauge transformation changing the sign of Fermi fields, see e.g. [23].

Theorem 3.14 Let $\mathcal{F}$ be a field net over $\mathcal{K}$ on a Hilbert space $\mathcal{H}$ satisfying twisted duality under a compact group of unitaries $G$ inducing automorphisms of the net $\mathcal{F}$. Let $\mathcal{H}$, the subspace of $G$-invariant vectors, be cyclic for each $\mathcal{F}(\mathcal{O})$. Then the fixed-point net $\mathcal{A}$ satifies duality for each $\mathcal{O} \in \mathcal{K}$ provided $\mathcal{O}^{\perp}$ is connected.

Proof. Let $E$ denote the projection onto $\mathcal{H}$ then the conditional expectation $m$ of $\mathcal{F}$ onto $\mathcal{A}$ may either be defined by integrating over the action of $G$ or by

$$
m(F) E=E F E, \quad F \in \mathcal{F} .
$$

Now

$$
\left(\mathcal{A}_{E}\right)^{d}(\mathcal{O})=\cap_{\mathcal{O}_{1} \perp \mathcal{O}}\left(\mathcal{A}_{E}\left(\mathcal{O}_{1}\right)^{\prime}\right)=\cap_{\mathcal{O}_{1} \perp \mathcal{O}}\left(E \mathcal{F}^{t}\left(\mathcal{O}_{1}\right) E \uparrow \mathcal{H}_{0}\right)^{\prime}
$$

Since $E$ is cyclic and separating for each $\mathcal{F}^{t}(\mathcal{O})$ and $\mathcal{A}(\mathcal{O})=m(\mathcal{F}(\mathcal{O}))$,

$$
\left(E \mathcal{F}^{t}\left(\mathcal{O}_{1}\right) E \uparrow \mathcal{H}_{0}\right)^{\prime}=\left(E \mathcal{F}^{t}\left(\mathcal{O}_{1}\right)^{\prime} E\right)\left\lceil\mathcal{H}_{0} .\right.
$$

Now using the fact that $E$ is separating for each $\mathcal{F}^{t}(\mathcal{O})^{\prime}$ and that $\mathcal{O}^{\perp}$ is pathconnected, we obtain

$$
\left(\mathcal{A}_{E}\right)^{d}(\mathcal{O})=E \cap_{\mathcal{O}_{1} \perp \mathcal{O}} \mathcal{F}^{t}\left(\mathcal{O}_{1}\right)^{\prime} E\left\lceil\mathcal{H}_{0}=\mathcal{A}(\mathcal{O}),\right.
$$

since $\mathcal{F}$ satisfies twisted duality.

What is still missing is a result allowing one to pass from the Borchers Property for the field net to the corresponding property of the observable net. 


\subsection{Appendix to Chapter 3}

In this Appendix, we begin by introducing various notions we shall need in connection with the partially ordered set $\mathcal{K}$. We recall 53 that an 0 -simplex $a$ of the partially ordered set $\mathcal{P}$ is just an element of $\mathcal{P}$ and a 1 -simplex $b$ consists of two 0 -simplices denoted $\partial_{0} b$ and $\partial_{1} b$ contained in a third element $|b|$ of $\mathcal{P}$ called the support of $b$. More generally, an $n$-simplex is an order-preserving map into $\mathcal{P}$ from the set of subsimplices of the standard $n$-simplex, ordered under inclusion. $\Sigma_{n}(\mathcal{P})$ or just $\Sigma_{n}$ will denote the partially ordered set of $n$-simplices of $\mathcal{P}$ with the pointwise ordering.

A partially ordered set $\mathcal{P}$ is connected if given $a, a^{\prime} \in \Sigma_{0}(\mathcal{P})$, there is a path from $a$ to $a^{\prime}$ in $\mathcal{P}$, i.e. if there exist $b_{0}, b_{1}, \ldots, b_{n} \in \Sigma_{1}(\mathcal{P})$ with $\partial_{0} b_{0}=a, \partial_{1} b_{n}=a^{\prime}$ and $\partial_{0} b_{i}=\partial_{1} b_{i-1}, i=1,2, \ldots, n$. Obviously, if $\mathcal{P}$ is not connected, it is a disjoint union of its connected components. We will be taking for $\mathcal{P}$ not only subsets of $\mathcal{K}$ with the induced order but also of $\mathcal{K} \times \mathcal{K}$ with the product ordering. These notions are related to topological notions in the following way.

Lemma 3A.1 Let $\mathcal{P}$ be a base for the topology of a space $M$ and ordered under inclusion and suppose the elements of $\mathcal{P}$ are open, (non-empty) and path-connected. Then an open subset $X$ of $M$ is path-connected if and only if $\mathcal{P}_{X}:=\{\mathcal{O} \in \mathcal{P}: \mathcal{O} \subset X\}$ is connected.

Proof. Any two points of $X$ are contained in elements of $\mathcal{P}_{X}$ so if this is connected and each of its elements are path-connected the two points can be joined by a path in $X$. Conversely, given $\mathcal{O}_{0}, \mathcal{O}_{1} \in \mathcal{P}_{X}$, there is a path in $X$ beginning in $\mathcal{O}_{1}$ and ending in $\mathcal{O}_{0}$, if $X$ is pathwise connected. Since $\mathcal{P}$ is a base for the topology, it is easy to construct a path in $\mathcal{P}_{X}$ joining $\mathcal{O}_{1}$ and $\mathcal{O}_{0}$.

A subset $\mathcal{S}$ of $\mathcal{P}$ of the form $\mathcal{P}_{X}$ has the property that $\mathcal{O} \in \mathcal{S}$ and $\mathcal{O}_{1} \subset \mathcal{O}$ implies $\mathcal{O}_{1} \in \mathcal{S}$. Such subsets are referred to as sieves. If $\mathcal{P}$ is a base for the topology of $M$ then a sieve $\mathcal{S}$ is a base for the topology of the open subset $X_{\mathcal{S}}:=\cup\{\mathcal{O}: \mathcal{O} \in \mathcal{S}\}$. The connected components of a partially ordered set are sieves, the union or intersection of sieves is again a sieve.

Corollary 3A.2 Under the hypotheses of Lemma 3A.1, the connected components of $\mathcal{P}$ are of the form $\mathcal{P}_{X}$, where $X$ runs over the path-connected components of $M$.

We let Open $(M)$ denote the set of open subsets of $M$ ordered under inclusion and $\operatorname{Sieve}(\mathcal{K})$ the set of sieves of $\mathcal{K}$, then defining for a open set $X$ of $M, \mu(X)$ to be the set of $\mathcal{O} \in \mathcal{K}$ contained in $X, \mu$ is an injective order-preserving map from $\operatorname{Open}(M)$ to $\operatorname{Sieve}(\mathcal{K})$. If we define $\nu(\mathcal{S}):=X_{\mathcal{S}}$, then $\nu$ is order-preserving and a left inverse for $\mu$.

The following result will prove useful in calculating the connected components of a partially ordered sets.

Lemma 3A.3 Let $i \mapsto \mathcal{P}_{i}$ be an order-preserving map from a partially ordered set I to the set of sieves of a partially ordered set $\mathcal{P}$ ordered under inclusion. Suppose that $\mathcal{P}=\cup_{i \in I} \mathcal{P}_{i}$. Let $\mathcal{C} \subset \mathcal{P}$ and set $\mathcal{C}_{i}:=\mathcal{C} \cap \mathcal{P}_{i}$ then $\mathcal{C}$ is a union of components of $\mathcal{P}$ if and only if $\mathrm{C}_{i}$ is a union of components of $\mathcal{P}_{i}$ for each $i \in I$. If $I$ is connected and $\mathfrak{C}_{i}$ is either empty or a component of $\mathcal{P}_{i}, i \in I$, then $\mathcal{C}$ is a component of $\mathcal{P}$. 
Proof. If $\mathcal{C}$ is a union of components and $b \in \Sigma_{1}\left(\mathcal{P}_{i}\right)$ with $\partial_{1} b \in C_{i}$ then $b \in \Sigma_{1}(\mathcal{P})$ so $\partial_{0} b \in \mathcal{C} \cap \mathcal{P}_{i}=\mathcal{C}_{i}$ and $\mathcal{C}_{i}$ is a union of components. Conversely, if each $\mathcal{C}_{i}$ is a union of components and $b \in \Sigma_{1}(\mathcal{P})$ with $\partial_{1} b \in \mathcal{C}$, then $|b| \in \mathcal{P}_{i}$ for some $i$. But $\mathcal{P}_{i}$ is a sieve so $b \in \Sigma_{1}\left(\mathcal{P}_{i}\right)$ and $\partial_{1} b \in \mathcal{C} \cap \mathcal{P}_{i}$. Since $\mathcal{C}_{i}$ is a union of components, $\partial_{0} b \in \mathcal{C}_{i} \subset \mathcal{C}$ so $\mathcal{C}$ is a union of components. Now $\mathcal{C}$ is a component, if any given pair $a \in \mathcal{C}_{i}$ and $a^{\prime} \in \mathcal{C}_{i^{\prime}}$ can be joined by a path in $\mathcal{C}$. But $I$ being connected, we may as well suppose $i$ and $i^{\prime}$ have an upper bound $j \in I$. If $\mathcal{C}_{j}$ is a component, $a$ and $a^{\prime}$ can even be joined by a path in $\mathcal{C}_{j}$, completing the proof of the lemma.

Now an automorphism $g$ of a partially ordered set $\mathcal{P}$ such that given $\mathcal{O} \in \mathcal{P}$ there is a $b \in \Sigma_{1}(\mathcal{P})$ with $\partial_{1} b \subset \mathcal{O}$ and $\partial_{0} b \subset g \mathcal{O}$ obviously leaves each connected component of $\mathcal{P}$ globally invariant. If $G$ is a connected topological group acting continuously on a topological space $M$ and $\mathcal{P}$ is a base for the topology of $M$, then it is easy to see that given $\mathcal{O} \in \mathcal{K}$ there is a $\mathcal{O}_{1} \in \mathcal{K}$ and a neighbourhood $\mathcal{N}$ of the unit in $G$ such that $\mathcal{N} \mathcal{O}_{1} \subset \mathcal{O}$. It follows that $G$ leaves any path-component of $\mathcal{P}$ globally invariant. Of course, this may also be deduced from Corollary 3A.2.

After these generalities on partially ordered sets, we turn to the theory of superselection sectors and need a partially ordered set $\mathcal{K}$ equipped with a binary relation $\perp$ satisfying a), b) and c) of Sec. 3.1. Note that b) just says that $\mathcal{O}^{\perp}$ is a sieve of $\mathcal{K}$. There are two derived binary relations $\tilde{\perp}$ and $\hat{\perp}$ defined by supplementing $\mathcal{O}_{1} \perp \mathcal{O}_{2}$ by requiring that there exists an $\mathcal{O}_{3} \in \mathcal{K}$ such that

$$
\mathcal{O}_{1} \perp \mathcal{O}_{3}, \quad \mathcal{O}_{2} \perp \mathcal{O}_{3}
$$

or such that

$$
\mathcal{O}_{1}, \mathcal{O}_{2} \subset \mathcal{O}_{3},
$$

respectively. These relations automatically satisfy a) and b) but c) remains to be checked and will not prove to be a problem in our applications to curved spacetime. The operation of passing from $\perp$ to $\tilde{\perp}$ or $\hat{\perp}$ is idempotent and if $\mathcal{K}$ is directed, all three relations coincide. Furthermore, by Lemma 5.6, the corresponding notions of duality coincide for additive nets when $\mathcal{K}$ is the set of regular diamonds in a globally hyperbolic spacetime.

If $\mathcal{O}_{1} \perp \mathcal{O}_{2}$ and $\mathcal{O}_{3}$ has non-trivial causal complement in $\mathcal{O}$, i.e. if there exists an $\mathcal{O}_{4}$ with $\mathcal{O}_{3} \perp \mathcal{O}_{4}, \mathcal{O}_{3}, \mathcal{O}_{4} \subset \mathcal{O}_{2}$ then trivially $\mathcal{O}_{1} \tilde{\perp} \mathcal{O}_{3}$. Now a regular diamond is a union of a sequence of smaller regular diamonds with non-trivial causal complement in the original regular diamond. Thus when $\mathcal{K}$ is the set of regular diamonds, the difference between the relations $\perp$ and $\tilde{\perp}$ is, in this sense, a boundary effect.

The difference between $\tilde{\perp}$ and $\hat{\perp}$ merely reflects the potential difficulty of finding suitably large regular diamonds. If we replace the set $\mathcal{K}$ of regular diamonds by the set $\tilde{\mathcal{K}}$ of sieves in $\mathcal{K}$ with non-trivial causal complement, defining the causal complement $\mathcal{S}^{\perp}$ of a sieve $\mathcal{S}$ to be the sieve $\mathcal{S}^{\perp}:=\cap_{\mathcal{O} \in \mathcal{S}} \mathcal{O}^{\perp}$, then $\tilde{\perp}=\hat{\perp}$. In fact, if $\mathcal{S}_{1} \tilde{\perp} \mathcal{S}_{2}$, then $\left(\mathcal{S}_{1} \cup \mathcal{S}_{2}\right)^{\perp}=\mathcal{S}_{1}^{\perp} \cap \mathcal{S}_{2}^{\perp} \neq \emptyset$ so that $\mathcal{S}_{1} \tilde{\perp} \mathcal{S}_{2}$.

If $\mathcal{K}$ is a base of open sets of a topological space $M$ and the relation $\perp$ on $\mathcal{K}$ is induced by a relation $\perp$ on $\operatorname{Open}(M)$ satisfying a) and b) of Sec. 3.1 and which is local in the sense that if $X \in \operatorname{Open}(M)$ and $X \subset \cup_{i} \mathcal{O}_{i}$, then $\mathcal{O}_{i} \perp \mathcal{O}$ for all $i$ implies 
$X \perp \mathcal{O}$. This condition is obviously satisfied by the relation of causal disjointness on a globally hyperbolic spacetime. It implies that $\mu\left(X^{\perp}\right)=\mu(X)^{\perp}$. We also have $\nu(\mathcal{S})^{\perp}=\nu\left(\mathcal{S}^{\perp}\right)$ for any sieve $\mathcal{S}$ in $\mathcal{K}$.

Lemma 3.A4 When restricted to causally closed open sets and sieves, the maps $\mu$ and $\nu$ are inverses of one another.

Proof. If $\mathcal{S}$ is a sieve and $X:=\nu(\mathcal{S})$, then $\mu(X)^{\perp}=\mathcal{S}^{\perp}$. If $\mathcal{S}$ is causally closed, so is $X$ since $\mu$ is injective. On the other hand, if $X$ is causally closed and we set $\mathcal{S}:=\mu(X)$, then $S^{\perp \perp}=\mu\left(X^{\perp \perp}\right)=\mu(X)$ and $\mathcal{S}$ is causally closed. It remains to show that $\mathcal{S}=\mu \nu(\mathcal{S})$ if $\mathcal{S}$ is causally closed. But, in this case,

$$
\mathcal{S} \subset \mu \nu(\mathcal{S}) \subset \mu \nu(\mathcal{S})^{\perp \perp}=\mathcal{S}^{\perp \perp}=\mathcal{S}
$$

completing the proof.

By a representation $\pi$ of a net of von Neumann algebras $\mathcal{A}$ over $\mathcal{K}$ we mean normal representations $\pi_{\mathcal{O}}$ of $\mathcal{A}(\mathcal{O})$ on a Hilbert space $\mathcal{H}_{\pi}$ such that $\pi_{\mathcal{O}_{1}}$ is $\pi_{\mathcal{O}_{2}}$ restricted to $\mathcal{A}\left(\mathcal{O}_{1}\right)$, whenever $\mathcal{O}_{1} \subset \mathcal{O}_{2}$ in $\mathcal{K}$.

If $G$ is a group of automorphisms of $\mathcal{K}$ and $(\mathcal{A}, \alpha)$ is a covariant net then a covariant representation is a pair $(\pi, U)$ consisting of a representation $\pi$ of $\mathcal{A}$ and a unitary representation of $G$ on $\mathcal{H}_{\pi}$ such that $U(g) \pi_{\mathcal{O}}(A)=\pi_{g \mathcal{O}}\left(\alpha_{g}(A) U(g), A \in \mathcal{A}(\mathcal{O}), g \in G\right.$.

We now provide a cohomological interpretation of superselection sectors leading to a proof of the Extension Theorem of Sec. 3.1. To enter into the spirit of the cohomological interpretation, we regard $\mathcal{O}^{\perp}, \mathcal{O} \in \mathcal{K}$ as being a covering of $\mathcal{K}$, the causal covering. The selection criterion selects those representations that are trivial on the causal cover and these representations allow a cohomological description in analogy with locally trivial bundles.

For each $a \in \Sigma_{0}$ we pick a unitary $V_{a}$ such that

$$
V_{a} \pi_{\mathcal{O}}(A)=A V_{a}, \quad A \in \mathcal{A}(\mathcal{O}), \quad \mathcal{O} \perp a
$$

and set $z(b):=V_{\partial_{0} b} V_{\partial_{1} b}^{*}, b \in \Sigma_{1}$. Obviously if $\mathcal{O} \in|b|^{\perp}, z(b) \in \mathcal{A}(\mathcal{O})^{\prime}$ thus $z(b) \in$ $\mathcal{A}^{d}(|b|)$. Furthermore,

$$
z\left(\partial_{0} c\right) z\left(\partial_{2} c\right)=z\left(\partial_{1} c\right), \quad c \in \Sigma_{2}
$$

so that $z$ is a unitary 1 -cocycle with values in the dual net $\mathcal{A}^{d}$. We consider such 1 -cocycles as objects of a category $Z^{1}\left(\mathcal{A}^{d}\right)$, where an arrow $t$ in this category from $z$ to $z^{\prime}$ is a $t_{a} \in \mathcal{A}^{d}(a), a \in \Sigma_{0}$, such that

$$
t_{\partial_{0} b} z(b)=z^{\prime}(b) t_{\partial_{1} b}, \quad b \in \Sigma_{1} .
$$

This makes $Z^{1}\left(\mathcal{A}^{d}\right)$ into a $W^{*}$-category. Note that $\left\|t_{a}\right\|$ is independent of $a$.

If we were to make a different choice $V_{a}^{\prime}$ of unitaries $V_{a}$, then setting $z^{\prime}(b):=$ $V_{\partial_{0} b}^{\prime} V_{\partial_{1} b}^{\prime *}$ and $w_{a}:=V_{a}^{\prime} V_{a}^{*}$, we see that $w_{a} \in \mathcal{A}^{d}(a)$ and $w_{\partial_{0} b} z(b)=z^{\prime}(b) w_{\partial_{1} b}$. Thus $w \in\left(z, z^{\prime}\right)$ is a unitary and the 1-cocycle attached to $\pi$ is defined up to unitary equivalence in $Z^{1}\left(\mathcal{A}^{d}\right)$. More generally, if $T \in\left(\pi, \pi^{\prime}\right)$ and $\pi$ and $\pi^{\prime}$ are trivial on the causal cover and $z$ and $z^{\prime}$ are associated cocycles defined by unitaries $V_{a}$ and $V_{a}^{\prime}$, as above, set

$$
t_{a}:=V_{a}^{\prime} T V_{a}^{*}, a \in \Sigma_{0}
$$


Then $t_{a} \in \mathcal{A}^{d}(a)$ and

$$
t_{\partial_{0} b} z(b)=V_{\partial_{0} b}^{\prime} T V_{\partial_{0} b}^{*} V_{\partial_{0} b} V_{\partial_{1} b}^{*}=V_{\partial_{0} b}^{\prime} T V_{\partial_{1} b}^{*}=V_{\partial_{0} b}^{\prime} V_{\partial_{1} b}^{\prime *} V_{\partial_{1} b}^{\prime} T V_{\partial_{0} b}^{*}=z^{\prime}(b) t_{\partial_{1} b},
$$

so that $t \in\left(z, z^{\prime}\right)$. Conversely, if $t \in\left(z, z^{\prime}\right)$ then $T:=V_{a}^{\prime *} t_{a} V_{a}$ is independent of $a$ so that

$$
T \pi_{\mathcal{O}}(A)=\pi_{\mathcal{O}}^{\prime}(A) T, \quad A \in \mathcal{A}(\mathcal{O}), \quad \mathcal{O} \in \mathcal{K} .
$$

and we clearly have a close relation between $Z^{1}\left(\mathcal{A}^{d}\right)$ and the $W^{*}$-category $\operatorname{Rep}{ }^{\perp} \mathcal{A}$ of representations of $\mathcal{A}$ trivial on the causal cover.

However, any cocycle $z$ arising from such a representation has two special properties that may not be shared by a general 1-cocycle. First, $z$ is trivial on $\mathcal{B}(\mathcal{H})$, i.e. there are unitaries $V_{a}, a \in \Sigma_{0}$, on $\mathcal{H}$ such that $z(b)=V_{\partial_{0} b} V_{\partial_{1} b}^{*}, b \in \Sigma_{1}$.

If $\mathcal{K}$ is directed then $\Sigma_{*}(\mathcal{K})$ admits a contracting homotopy 53 . In this case every 1 -cocycle of $\mathcal{A}^{d}$ is trivial in $\mathcal{B}\left(\mathcal{H}_{0}\right)$. In general, if we consider the graph with vertices $\Sigma_{0}$ and arrows $\Sigma_{1}$ then the category generated by this graph has as arrows the paths in $\mathcal{K}$. Thus every 1 -cocycle extends to a functor from this category. When $z$ is trivial on $\mathcal{B}\left(\mathcal{H}_{0}\right)$ then $z(p)$ for a path $p$ depends only on the endpoints $\partial_{0} p$ and $\partial_{1} p$ of the path. Conversely, if $z(p)$ just depends on the endpoints of $p$ and $\mathcal{K}$ is connected, then $z$ is trivial on $\mathcal{B}\left(\mathcal{H}_{0}\right)$. To see this we pick a base point $a_{0} \in \Sigma_{0}$, then given $a \in \Sigma_{0}$ a path $p_{a}$ with $\partial_{0} p_{a}=a$ and $\partial_{1} p_{a}=a_{0}$ and finally define $y(a)=z\left(p_{a}\right)$. $z(p) y\left(\partial_{1} p\right)=y\left(\partial_{0} p\right)$, so we have trivialized $z$ in $\mathcal{B}\left(\mathcal{H}_{0}\right)$.

Secondly, for any path $p, z(p) A z(p)^{*}=A$ whenever $A \in \mathcal{A}(\mathcal{O})$ and $\partial_{0} p, \partial_{1} p \in \mathcal{O}^{\perp}$. The full subcategory of $Z^{1}\left(\mathcal{A}^{d}\right)$ whose objects satisfy these two conditions will be denoted by $Z_{t}^{1}\left(\mathcal{A}^{d}\right)$.

The following simple result shows that the second condition is automatically satisfied in an important special case.

Lemma 3A.5 If $\mathcal{O}^{\perp}$ is connected, then any object $z$ of $Z^{1}\left(\mathcal{A}^{d}\right)$, trivial on $\mathcal{B}\left(\mathcal{H}_{0}\right)$ satisfies

$$
z(p) A z(p)^{*}=A, \quad \partial_{0} p, \partial_{1} p \in \mathcal{O}^{\perp}, A \in \mathcal{A}(\mathcal{O}) .
$$

Proof. Since $\mathcal{O}^{\perp}$ is connected, it suffices to prove the result when the path $p$ is a 1 -simplex $b$ with $|b| \in \mathcal{O}^{\perp}$. But then, $z(b) \in \mathcal{A}^{d}(|b|) \subset \mathcal{A}(\mathcal{O})^{\prime}$.

Having discussed these two conditions, we can give our cohomological characterization of the selection criterion.

Theorem 3A.6 The $W^{*}$-categories $\operatorname{Rep}^{\perp} \mathcal{A}$ and $Z_{t}^{1}\left(\mathcal{A}^{d}\right)$ are equivalent.

Proof. We pick unitaries $V_{a}^{\pi}, a \in \Sigma_{0}$, as above, for each object $\pi$ of $\operatorname{Rep}^{\perp} \mathcal{A}$. Given an arrow $T \in\left(\pi, \pi^{\prime}\right)$ in that category, we define for $b \in \Sigma_{1}, a \in \Sigma_{0}$

$$
F(\pi)(b)=V_{\partial_{0} b}^{\pi} V_{\partial_{1} b}^{\pi *} ; \quad F(T)_{a}:=V_{a}^{\pi^{\prime}} T V_{a}^{\pi *} .
$$

Then $F$ is a faithful *-functor and our computations above show that it is full. Hence, it remains to show that each object $z$ of $Z_{t}^{1}\left(\mathcal{A}^{d}\right)$, is equivalent to an object in the 
image of $F$. We show this by constructing a representation $\pi^{z}$. We pick unitaries $V_{a}$, $a \in \Sigma_{0}$, on $\mathcal{H}_{0}$ such that $z(b)=V_{\partial_{0} b} V_{\partial_{1} b}^{*}, \quad b \in \Sigma_{1}$, and define

$$
\pi_{\mathcal{O}}^{z}(A)=V_{a}^{*} A V_{a}, \quad a \in \mathcal{O}^{\perp}, \quad A \in \mathcal{A}(\mathcal{O}) .
$$

This is well defined since $\mathcal{K}$ is connected and for any path $p$ with $\partial_{0} p, \partial_{1} p \in \mathcal{O}^{\perp}$ we have $z(p) \in \mathcal{A}(\mathcal{O})^{\prime}$. Furthermore, the definition respects the net structure since

$$
\pi_{\mathcal{O}_{1}}^{z}(A)=\pi_{\mathcal{O}_{2}}^{z}(A), \quad A \in \mathcal{A}\left(\mathcal{O}_{1}\right), \quad \mathcal{O}_{1} \subset \mathcal{O}_{2} .
$$

Hence we get a representation of the net $\mathcal{A}$, trivial on the covering by construction and $V_{\partial_{0} b} V_{\partial_{1} b}^{*}=z(b)$ is an associated 1-cocycle. This completes the proof.

We now consider the problem of extending representations of a net $\mathcal{A}$, trivial on the causal cover, to representations of the bidual net $\mathcal{A}^{d d}$, again trivial on the causal cover.

Theorem 3A.7 If each $\mathcal{O}^{\perp}$ is connected, every object $\pi$ of $\operatorname{Rep}^{\perp} \mathcal{A}$ admits a unique extension to an object of $\operatorname{Rep}^{\perp} \mathcal{A}^{\text {dd }}$. Furthermore there is a canonical isomorphism of $W^{*}$-categories $\operatorname{Rep}^{\perp} \mathcal{A}$ and $\operatorname{Rep}^{\perp} \mathcal{A}^{d d}$.

Proof. Let $V_{a}, a \in \Sigma_{0}$ be unitaries realizing the equivalence of $\pi$ and $\pi^{0}$ on $a^{\perp}$. Then $z(b):=V_{\partial_{0} b} V_{\partial_{1} b}^{*}, b \in \Sigma_{1}$ is an associated object of $Z_{t}^{1}\left(\mathcal{A}^{d}\right)$. Since each $\mathcal{O}^{\perp}$ is connected, $z$ is at the same time an object of $Z^{1}\left(\mathcal{A}^{\text {ddd }}\right)$ by Lemma 3.A.4. If we define

$$
\tilde{\pi}_{\mathcal{O}}(A):=V_{a}^{*} A V_{a}, \quad A \in \mathcal{A}^{d d}(\mathcal{O}), \quad a \in \mathcal{O}^{\perp},
$$

this gives a well defined element of $\operatorname{Rep}^{\perp} \mathcal{A}^{d d}$ just as in the proof of Theorem 3A.6. Furthermore, $\tilde{\pi}$ obviously extends $\pi$ by the choice of the $V_{a}$. If we make another choice $V_{a}^{\prime}$ of the $V_{a}$ then $V_{a}^{\prime} V_{a}^{*} \in \mathcal{A}^{d}(a)$ so that $\tilde{\pi}$ remains unchanged and is consequently the unique extension of $\pi$ to an object of $\operatorname{Rep}^{\perp} \mathcal{A}^{d d}$. Passing to the extensions does not change the intertwiners by Theorem 3A.6.

For the further development of superselection theory, we must assume duality $\mathcal{A}=\mathcal{A}^{d}$, although essential duality would do whenever each $\mathcal{O}^{\perp}$ is connected. We shall even need to assume $\tilde{\perp}$-duality, but this coincides with duality in curved spacetime whose status is commented on in Sec. 4.2.

The next goal is to show that sectors have a tensor structure. More precisely, we shall show that $Z^{1}(\mathcal{A})$ has a canonical structure of a tensor $W^{*}$-category arising by adjoining endomorphisms. If $\mathcal{A}$ is a net of von Neumann algebras, then there is an associated net $\mathcal{O} \mapsto \operatorname{End} \mathcal{A}(\mathcal{O})$ of tensor $W^{*}$-categories. End $\mathcal{A}(\mathcal{O})$ has as objects the normal endomorphisms of the net $\mathcal{O}_{1} \mapsto \mathcal{A}\left(\mathcal{O}_{1}\right)$, i.e. normal endomorphisms $\rho_{\mathcal{O}_{1}}$ of $\mathcal{A}\left(\mathcal{O}_{1}\right)$ compatible with the net structure. An arrow $T \in(\rho, \sigma)$ in $\operatorname{End} \mathcal{A}(\mathcal{O})$ is a $T \in \mathcal{A}(\mathcal{O})$ such that

$$
T \rho(A)=\sigma(A) T, \quad A \in \mathcal{A}\left(\mathcal{O}_{1}\right), \mathcal{O} \subset \mathcal{O}_{1} .
$$

The tensor structure is defined on the lines of Sec. 3.3 and the net structure is given by the obvious restriction mappings. 
The construction of appropriate endomorphisms is just a variant on that already used to pass from a 1 -cocycle $z \in Z_{t}^{1}\left(\mathcal{A}^{d}\right)$ to a representation $\pi^{z}$. Given $a \in \Sigma_{0}$, and $A \in \mathcal{A}(\mathcal{O}), a \subset \mathcal{O}$ pick a path $p$ with $\partial_{0} p=a$ and $\partial_{1} p \in \mathcal{O}^{\perp}$ and set

$$
y(a)(A):=z(p) A z(p)^{*} .
$$

$y(a)(A)$ is independent of the choice of $p$ since $z \in Z_{t}^{1}\left(\mathcal{A}^{d}\right)$. Given $X \in \mathcal{A}\left(\mathcal{O}_{1}\right)$ with $\mathcal{O}_{1} \perp \mathcal{O}, \mathcal{O}_{2}$ with $\mathcal{O}_{2} \perp \mathcal{O}$ and $\mathcal{O}_{2} \perp \mathcal{O}_{1}$ and choosing $\partial_{1} p=\mathcal{O}_{2}$, we see that $y(a)(A)$ and $X$ commute so that $y(a)(A) \in \mathcal{A}(\mathcal{O})$ by $\tilde{\perp}$-duality. Thus $y(a)$ is an object of $\operatorname{End} \mathcal{A}(a)$.

But $y(a)$ is not only localized in $a$ in the sense of net automorphisms but also in the sense of superselection theory in that $y(a)(A)=A$ whenever $A \in \mathcal{A}\left(\mathcal{O}_{1}\right)$ where $\mathcal{O}_{1} \in a^{\perp}$ and $\mathcal{O}_{1}, a \subset \mathcal{O}$, since the endpoints of $p$ lie in $\mathcal{O}_{1}^{\perp}$. We write $\Delta(a)$ to denote the objects of End $\mathcal{A}(a)$ satisfying this second localization condition and denote by $\mathcal{T}(a)$ the corresponding full tensor $C^{*}$-subcategory of $\operatorname{End} \mathcal{A}(a)$.

Lemma 3A.8 Let $p$ be a path with $\partial_{1} p, \partial_{0} p \subset \mathcal{O}$ then

$$
z(p) y\left(\partial_{1} p\right)(A)=y\left(\partial_{0} p\right)(A) z(p), \quad A \in \mathcal{A}(\mathcal{O}) .
$$

Proof. Given $A \in \mathcal{A}(\mathcal{O})$ and a path $p$ with $\partial_{1} p, \partial_{0} p \subset \mathcal{O}$, pick paths $p^{\prime}, p^{\prime \prime}$ with $\partial_{0} p^{\prime}=\partial_{1} p, \partial_{0} p^{\prime \prime}=\partial_{0} p$ and $\partial_{1} p^{\prime}, \partial_{1} p^{\prime \prime} \in \mathcal{O}^{\perp}$, then

$$
z(p) y\left(\partial_{1} p\right)(A)=z(p) z\left(p^{\prime}\right) A z\left(p^{\prime}\right)^{*}=z\left(p^{\prime \prime}\right) A z\left(p^{\prime \prime}\right)^{*} z(p)=y\left(\partial_{0} p\right)(A) z(p),
$$

as required.

Furthermore if $t \in(z, \hat{z}), A \in \mathcal{A}(\mathcal{O})$ and $p$ is a path with $\partial_{0} p=a \subset \mathcal{O}$ and $\partial_{1} p \subset \mathcal{O}^{\perp}$ then

$$
t_{a} y(a)(A)=t_{a} z(p) X z(p)^{*}=\hat{z}(p) t_{\partial_{1} p} A z(p)^{*}=\hat{z}(p) A \hat{z}(p)^{*} t_{a}=\hat{y}(a)(A) t_{a} .
$$

In other words $t_{a} \in(y(a), \hat{y}(a))$.

These results admit the following interpretation.

Theorem 3A.9 Let $\mathcal{A}$ be a net over $(\mathcal{K}, \perp)$ satisfying $\tilde{\perp}$-duality. If $z$ is a 1 -cocycle of $\mathcal{A}$ trivial in $\mathcal{B}\left(\mathcal{H}_{0}\right)$ then $(y, z)$ is a 1 -cocycle in the net $\mathcal{T}$ of tensor $W^{*}$-categories and the map $z \mapsto(y, z)$ together with the identity map on arrows is an isomorphism of $Z_{t}^{1}(\mathcal{A})$ and $Z_{t}^{1}(\mathcal{T})$.

Now, $\mathcal{T}$ being a net of tensor $W^{*}$-categories, $Z^{1}(\mathcal{T})$ is itself a tensor $W^{*}$-category. Given 1-cocycles $\left(y_{1}, z_{1}\right)$ and $\left(y_{2}, z_{2}\right)$, their tensor product is the 1-cocycle $(y, z)$, where

$$
y(a)=y_{1}(a) y_{2}(a), \quad z(b)=z_{1}(b) y_{1}\left(\partial_{1} b\right)\left(z_{2}(b)\right) .
$$

If both $\left(y_{1}, z_{1}\right)$ and $\left(y_{2}, z_{2}\right)$ are trivial in $\mathcal{B}\left(\mathcal{H}_{0}\right)$ then so is their tensor product. The tensor product on arrows is defined as follows: if $t_{i}$ maps from $\left(y_{i}, z_{i}\right)$ to $\left(y_{i}^{\prime}, z_{i}^{\prime}\right)$ for $i=1,2$, then the tensor product $t_{1} \otimes t_{2}$ is given by

$$
\left(t_{1} \otimes t_{2}\right)_{a}=t_{1, a} y_{1}(a)\left(t_{2, a}\right) .
$$


This completes our goal of describing superselection structure in terms of a tensor $W^{*}$-category. Note that we could have used the subnet $\mathcal{T}_{t}$ in place of $\mathcal{T}$ defined by requiring an object $\rho$ of $\mathcal{T}(\mathcal{O})$ to be transportable, i.e. there exists a map $a \mapsto \rho_{a}$, where $\rho_{a}$ is an object of $\mathcal{T}(a)$ and $\rho_{a}=\rho$ when $a=\mathcal{O}$ and a map $\Sigma_{1} \ni b \mapsto u(b)$, where $u(b)$ is an arrow from $\rho_{\partial_{1} b}$ to $\rho_{\partial_{0} b}$ in $\mathcal{T}(|b|)$. In fact the tensor $W^{*}$-categories $Z_{t}^{1}(\mathcal{T})$ and $Z_{t}^{1}\left(\mathcal{T}_{t}\right)$ are canonically isomorphic. In Sec. 3.3, we show how to get a net $\left(\mathcal{T}_{t}, \varepsilon_{c}\right)$ of braided tensor $W^{*}$-categories and it is a simple general fact that this leads to a braided tensor $W^{*}$-category, $\left(Z_{t}^{1}\left(\mathcal{T}_{t}\right), \varepsilon_{c}\right)$. We need only set

$$
\varepsilon_{c}\left(z, z^{\prime}\right)_{a}:=\varepsilon\left(y(a), y^{\prime}(a)\right) .
$$

Since this expression obviously acts correctly on the arrows evaluated in $a$ and the laws for a braiding hold for each $a$, the only point that has to be checked is that $\varepsilon\left(z, z^{\prime}\right)$ is an arrow from $z \times z^{\prime}$ to $z^{\prime} \times z$. However, if $b \in \Sigma_{1}, z(b) \in\left(\rho_{\partial_{1} b}, \rho_{\partial_{0} b}\right)$ in $\mathcal{T}_{t}(|b|)$ and similarly for $z^{\prime}(b)$. Thus

$$
z^{\prime}(b) \times z(b) \circ \varepsilon\left(\rho_{\partial_{1} b}, \rho_{\partial_{1} b}^{\prime}\right)=\varepsilon\left(\rho_{\partial_{0} b}, \rho_{\partial_{0} b}^{\prime}\right) \circ z(b) \times z^{\prime}(b)
$$

as required.

Thus the cohomological approach leads to a braided tensor $W^{*}$-category $\left(Z_{t}^{1}\left(\mathcal{T}_{t}\right)\right.$, $\left.\epsilon_{c}\right)$ describing superselection structure and in the context of globally hyperbolic spacetimes this is even a symmetric tensor $W^{*}$-category for spacetime dimensions $\geq 2$. It should be noted that except when $\mathcal{K}$ is directed, we have not given a direct description of this structure in terms of transportable localized endomorphisms. In particular, it not clear that every transportable localized endomorphism arises from a 1-cocycle. Furthermore, if $\rho$ and $\sigma$ are in $\Delta_{t}(\mathcal{O})$ and $T$ is a bounded operator on the ambient Hilbert space, such that

$$
T \rho(A)=\sigma(A) T, \quad A \in \mathcal{A}\left(\mathcal{O}_{1}\right), \mathcal{O} \subset \mathcal{O}_{1},
$$

then $T$ commutes with $\mathcal{A}\left(\mathcal{O}_{2}\right)$ for $\mathcal{O}_{2} \perp \mathcal{O}$ provided there is a $\mathcal{O}_{1}$ with $\mathcal{O}, \mathcal{O}_{2} \subset$ $\mathcal{O}_{1}$. This means, we would need duality with respect to the modified relation $\hat{\perp}$ to be able to conclude that $T \in \mathcal{A}(\mathcal{O})$ and hence that $T$ is an arrow from $\rho$ to $\sigma$ in $\mathcal{T}_{t}(\mathcal{O})$. Conversely, if $\pi$ and $\pi^{\prime}$ are representations satisfying the selection criterion and restricting to endomorphisms $\rho$ and $\rho^{\prime}$ in $\Delta_{t}(\mathcal{O})$ then it is not clear that an arrow $T \in\left(\rho, \rho^{\prime}\right)$ in $\mathcal{T}_{t}(\mathcal{O})$ will at the same time intertwine $\pi$ and $\pi^{\prime}$.

These points should be bourne in mind, when, in the main body of the text, we avoid the cohomological description and put the emphasis on transportable localized endomorphisms.

To proceed with the analysis of statistics, we need to use left inverses and we examine, at this point, the notions involved and the relations between them. If $\pi$ is a representation of $\mathcal{A}$ on $\mathcal{H}_{0}$ then we define a left inverse $\phi$ of $\pi$ to be given by unital positive linear mappings $\phi_{\mathcal{O}}$ on $\mathcal{B}\left(\mathcal{H}_{0}\right)$ compatible with the net inclusions and satisfying

$$
\phi_{\mathcal{O}}\left(A \pi_{\mathcal{O}}(B)\right)=\phi_{\mathcal{O}}(A) B, \quad A, B \in \mathcal{A}(\mathcal{O}) .
$$


Note that if $\pi_{\mathcal{O}}(B)=B$ then $\phi_{\mathcal{O}}(B)=B$. If $\pi$ is localized in $\mathcal{O}$ in the sense that

$$
\pi_{\mathcal{O}_{1}}(A)=A, \quad \mathcal{O} \perp \mathcal{O}_{1}, \quad A \in \mathcal{A}\left(\mathcal{O}_{1}\right),
$$

then $\phi$ is localized in $\mathcal{O}$ in the same sense. Furthermore, if $\mathcal{O}_{1} \hat{\perp} \mathcal{O}_{2}$ and $\mathcal{O} \subset \mathcal{O}_{2}$, then $\phi_{\mathcal{O}_{2}}(A) B=B \phi_{\mathcal{O}_{2}}(A)$ for $A \in \mathcal{A}\left(\mathcal{O}_{2}\right)$ and $B \in \mathcal{A}\left(\mathcal{O}_{1}\right)$. In fact, picking $\mathcal{O}_{3}$ with $\mathcal{O}_{1}, \mathcal{O}_{2} \subset \mathcal{O}_{3}$ we have

$$
\begin{gathered}
\phi_{\mathcal{O}_{2}}(A) B=\phi_{\mathcal{O}_{3}}(A) B=\phi_{\mathcal{O}_{3}}\left(A \pi_{\mathcal{O}_{3}}(B)\right) \\
=\phi_{\mathcal{O}_{3}}\left(A \pi_{\mathcal{O}_{1}}(B)\right)=\phi_{\mathcal{O}_{3}}(A B) .
\end{gathered}
$$

Since $A$ and $B$ commute, we interchange them and reverse the steps to conclude that $\phi_{\mathrm{O}_{2}}(A)$ and $B$ commute. This proves the following result.

Lemma 3A.10 If $\phi$ is a left inverse for a representation $\pi$ localized in $\mathcal{O}$ then $\phi$ is localized in $\mathcal{O}$ and if duality holds for the relation $\hat{\perp}, \phi_{\mathcal{O}_{1}} \mathcal{A}\left(\mathcal{O}_{1}\right) \subset \mathcal{A}\left(\mathcal{O}_{1}\right)$ for $\mathcal{O} \subset \mathcal{O}_{1}$.

The restriction of $\pi$ to the net $\mathcal{O}_{1} \mapsto \mathcal{A}\left(\mathcal{O}_{1}\right), \mathcal{O}_{1} \supset \mathcal{O}$ is a localized endomorphism $\rho$ and a object of the tensor $W^{*}$-category $\operatorname{End} \mathcal{A}(\mathcal{O})$. The above notion of left inverse adapts easily to localized endomorphisms. If $\rho$ is localized in $\mathcal{O}$, a left inverse of $\rho$ is a family $\mathcal{O}_{1} \supset \mathcal{O} \mapsto \phi_{\mathcal{O}_{1}}$ of unital positive linear mappings on the $\mathcal{A}\left(\mathcal{O}_{1}\right)$, compatible with the net inclusions and satisfying

$$
\phi_{\mathcal{O}_{1}}\left(A \rho_{\mathcal{O}_{1}}(B)\right)=\phi_{\mathcal{O}_{1}}(A) B, \quad A, B \in \mathcal{A}\left(\mathcal{O}_{1}\right) .
$$

Obviously, a left inverse for $\rho$ considered as a representation yields a left inverse for the endomorphism $\rho$ on restriction. If $\bar{\rho}$ is a conjugate for $\rho$ then we get a left inverse $\phi$ for $\rho$ by setting

$$
\phi_{\mathcal{O}_{1}}(A):=V^{*} \bar{\rho}_{\mathcal{O}_{1}}(A) V, \quad A \in \mathcal{A}\left(\mathcal{O}_{1}\right), \quad \mathcal{O}_{1} \supset \mathcal{O},
$$

where $V \in(\mathrm{id}, \bar{\rho} \rho)$ is an isometry.

The restriction of $\pi$ to the net $\mathcal{O}_{1} \mapsto \mathcal{A}\left(\mathcal{O}_{1}\right), \mathcal{O}_{1} \supset \mathcal{O}$ is a localized endomorphism $\rho$ and a object of the tensor $W^{*}$-category $\operatorname{End} \mathcal{A}(\mathcal{O})$. We now show that a left inverse $\phi$ for $\rho$ induces a left inverse of $\rho$ in the categorical sense 47]. In other words, we need a set

$$
\phi_{\sigma, \tau}:(\rho \sigma, \rho \tau) \rightarrow(\sigma, \tau),
$$

of linear mappings where $\sigma, \tau$ are objects of the category. These have to be natural in $\sigma$ and $\tau$, i.e. given $S \in\left(\sigma, \sigma^{\prime}\right)$ and $T \in\left(\tau, \tau^{\prime}\right)$ we have

$$
\phi_{\sigma^{\prime}, \tau^{\prime}}\left(1_{\rho} \otimes T \circ X \circ 1_{\rho} \otimes S^{*}\right)=T \circ \phi_{\sigma, \tau}(X) \circ S^{*}, X \in(\rho \sigma, \rho \tau),
$$

and furthermore to satisfy

$$
\phi_{\sigma \nu, \tau \nu}\left(X \otimes 1_{\pi}\right)=\phi_{\sigma, \tau}(X) \otimes 1_{\nu}, X \in(\rho \sigma, \rho \tau)
$$

for each object $\nu$. We will require that $\phi$ is positive in the sense that $\phi_{\sigma, \sigma}$ is positive for each $\sigma$ and normalized in the sense that

$$
\phi_{\iota, \iota}\left(1_{\rho}\right)=1_{\iota}
$$


We say that $\phi$ is faithful if $\phi_{\sigma, \sigma}$ is faithful for each object $\sigma$.

Now, given $T \in(\rho \sigma, \rho \tau)$, we recall that $T \in \mathcal{A}(\mathcal{O})$. Hence we set

$$
\phi_{\sigma, \tau}(T)=\phi_{\mathcal{O}}(T)
$$

and since $\phi_{\mathcal{O}}(T) \in \mathcal{A}(\mathcal{O})$ by Lemma 3A.9, we conclude without difficulty that we get a left inverse for $\rho$ in this way.

On the other hand, if we are dealing with a representation satisfying the selection criterion then we know that, by passing to an associated 1-cocycle, we get a field $a \mapsto y(a)$ of localized endomorphisms under the weaker assumption that duality holds for the relation $\tilde{\perp}$. In this case, we would actually like a left inverse for the 1 -cocycle considered as an object of the tensor $W^{*}$-category $Z_{t}^{1}(\mathcal{A})$. To this end, we pick, for each of the associated endomorphisms $y(a)$ a left inverse $\phi_{a}$ and ask whether $a \mapsto \phi_{a}\left(t_{a}\right)$ is an arrow from $z^{\prime}$ to $z^{\prime \prime}$, whenever $a \mapsto t_{a}$ is an arrow from $z \times z^{\prime}$ to $z \times z^{\prime \prime}$. Thus $t_{a} \in \mathcal{A}(a)$ and

$$
\left(z \times z^{\prime \prime}\right)(b) t_{\partial_{1} b}=t_{\partial_{0} b}\left(z \times z^{\prime}\right)(b)
$$

It follows that

$$
z^{\prime \prime}(b) \phi_{\partial_{1} b}\left(t_{\partial_{1} b}\right)=\phi_{\partial_{1} b}\left(y\left(\partial_{1} b\right)\left(z^{\prime \prime}(b)\right) t_{\partial_{1} b}\right)=\phi_{\partial_{1} b}\left(z(b)^{*} t_{\partial_{0} b} z(b)\right) z^{\prime}(b)
$$

and we deduce the following lemma.

Lemma 3A.11 If $z \in Z_{t}^{1}(\mathcal{A})$ and $a \mapsto y(a)$ is the associated field of endomorphisms. Then a field $a \mapsto \phi_{a}$ of left inverses of the $y(a)$ defines a left inverse for $z$ by the formula

$$
\phi_{z^{\prime}, z^{\prime \prime}}(t)_{a}:=\phi_{a}\left(t_{a}\right)
$$

provided $\phi_{\partial_{0} b}=\phi_{\partial_{1} b} \operatorname{Ad} z(b)^{*}$ for $b \in \Sigma_{1}$.

There is no a priori reason to suppose that every left inverse for a 1-cocycle arises from such a field of left inverses. In particular a map $t \in\left(z, z^{\prime}\right) \mapsto t_{a} \in\left(y(a), y^{\prime}(a)\right)$ might not be surjective. We can also not just begin with a left inverse $\phi_{a}$ for $y(a)$ since it is not clear that we get a field of left inverses using the cocycle. However, if we assume, as in Sec. 3.4, that $\mathcal{K}$ has an asymptotically causally disjoint net $\mathcal{O}_{n}$, then we can construct left inverses for 1-cocycles. If $z$ is an object of $Z_{t}^{1}(\mathcal{A})$, we denote by $z(a, n)$, the evaluation of $z$ on a path $p$ with $\partial_{0} b=a$ and $\partial_{1} b=\mathcal{O}_{n}$. This is independent of the chosen path. We now define $\phi_{a}(X)$ to be a Banach-limit over $n$ of $z(a, n)^{*} X z(a, n)$. Then $\phi_{a}$ is a positive linear map satisfying

$$
\phi_{a}(X) A=\phi_{a}\left(X \pi_{\mathcal{O}}^{a}(A)\right), \quad A \in \mathcal{A}(\mathcal{O}) .
$$

Furthermore, from the cocycle identity we have

$$
\phi_{\partial_{0} b}=\phi_{\partial_{1} b} \operatorname{Ad}(z(b))
$$

Since each $\phi_{a}$ defines a left inverse for $y(a)$, we have constructed a left inverse for $z$ by Lemma 3A.11. 
One sometimes wishes to consider nets defined over a wider class of regions than say just the set of regular diamonds. Thus in Sections 4 and 5, we are interested in defining the von Neumann algebras of wedge regions. Furthermore, another reason for wanting von Neumann algebras associated with large rather than small regions is that we can only compose endomorphisms if we find a joint localization region for the endomorphisms involved. We consider here the task of extending the domain of definition of the net in the context of the present formalism where $\mathcal{K}$ is a partially ordered set commenting on the relation with regions of spacetime afterwards. Thus instead of a region, we use the notion of a sieve $\mathcal{S}$, see above, and consider the set $\tilde{\mathcal{K}}$ of sieves $\mathcal{S}$ of $\mathcal{K}$ such that neither $\mathcal{S}$ nor $\mathcal{S}^{\perp}$ are the empty set, ordered under inclusion. To each such sieve $\mathcal{S}$, we associate the von Neumann algebra $\mathcal{A}(\mathcal{S})$ generated by the $\mathcal{A}(\mathcal{O})$ with $\mathcal{O} \in \mathcal{S}$ in the defining representation.

We now show that a representation $\pi$ of $\mathcal{A}$ satisfying the selection criterion has a natural extension to a representation of the net $\mathcal{S} \mapsto \mathcal{A}(\mathcal{S})$. We pick for each $a \in \Sigma_{0}$ a unitary $V_{a}$ such that

$$
\pi_{\mathcal{O}}(A)=V_{a}^{*} A V_{a}, \quad A \in \mathcal{A}(\mathcal{O}), \quad \mathcal{O} \in a^{\perp}
$$

and then define

$$
\pi_{\mathcal{S}}(A):=V_{a}^{*} A V_{a}, \quad A \in \mathcal{A}(\mathcal{S}), a \in \mathcal{S}^{\perp} .
$$

Note that this expression is well defined being independent of the choice of $a \in \mathcal{S}^{\perp}$ since if $a^{\prime} \in \mathcal{S}^{\perp}$ then

$$
V_{a^{\prime}} V_{a}^{*} \in \cap_{\mathcal{O} \in \mathcal{S}} \mathcal{A}(\mathcal{O})^{\prime}=\mathcal{A}(\mathcal{S})^{\prime} .
$$

In the same way, we see that $\pi_{\mathcal{S}}$ is independent of the choice of $a \mapsto V_{a}$. Note, too that we get a representation of the extended net in that if $\mathcal{S}_{1} \subset \mathcal{S}_{2}$ then $\pi_{\mathcal{S}_{1}}$ is the restriction of $\pi_{\mathcal{S}_{2}}$ to $\mathcal{A}\left(\mathcal{S}_{1}\right)$. Obviously, an intertwiner $T \in\left(\pi, \pi^{\prime}\right)$ over $\mathcal{K}$ remains and intertwiner over $\tilde{\mathcal{K}}$ so that effectively $\operatorname{Rep}^{\perp} \mathcal{A}$ remains unchanged when we extend the net.

That part of the formalism related to the concept of localized endomorphism is however sensitive to exteding the net. Although localized endomorphisms do not play the same fundamental role as 1-cocycles, we have found it convenient to use them in developing the theory. The problems involved in using them are two: they are not defined on the whole net and the natural map $\left(z, z^{\prime}\right) \mapsto\left(y(a), y^{\prime}(a)\right)$ may not be surjective. Extending the net improves matters in that localized endomorphisms are then defined on more operators and hence have fewer intertwiners. Since localized endomorphisms require subsets satisfying $\hat{\perp}$-duality, we benefit from the equality $\tilde{\perp}=\hat{\perp}$ on $\tilde{\mathcal{K}}$.

Supposing we have as usual a field $a \mapsto y(a), a \in \Sigma_{0}$, of localized endomorphisms derived from a 1-cocycle, then we know that if $\mathcal{O} \tilde{\perp} \mathcal{O}_{1}$ and $a \perp \mathcal{O}_{1}, y(a)(\mathcal{A}(\mathcal{O})) \subset$ $\mathcal{A}\left(\mathcal{O}_{1}\right)^{\prime}$. Hence $y(a)(\mathcal{A}(\mathcal{S})) \subset \cap_{\mathcal{O}_{1} \in \mathcal{S}^{\tilde{I}}} \mathcal{A}\left(\mathcal{O}_{1}\right)^{\prime}$. We conclude that if $\tilde{\perp}$-duality holds for $\mathcal{S}$ in the defining representation in the sense that

$$
\mathcal{A}(\mathcal{S})=\mathcal{A}\left(\mathcal{S}^{\tilde{\perp}}\right)^{\prime}
$$


then $y(a)$ acts as an endomorphism of $\mathcal{A}(\mathcal{S}), y(a)(\mathcal{A}(\mathcal{S})) \subset \mathcal{A}(\mathcal{S})$. Now if $\mathcal{S}$ satisfies $\tilde{\perp}$-duality then so does $\mathcal{S}^{\tilde{\perp}}$. Furthermore, $\mathcal{A}(\mathcal{S})=\mathcal{A}\left(\mathcal{S}^{\perp}\right)^{\prime} \supset \mathcal{A}\left(\mathcal{S}^{\perp \perp}\right)$. Thus $\mathcal{A}(\mathcal{S})=$ $\mathcal{A}\left(\mathcal{S}^{\perp \perp}\right)$. Hence, we may as well restrict attention to causally closed sieves and choose as our index set the set $\mathcal{L}$ of non-trivial causally closed sieves $\mathcal{S}$ for which $\tilde{\perp}$-duality holds either for $\mathcal{S}$ or for $\mathcal{S}^{\perp}$. This choice has the disadvantage of depending on the theory under consideration but it allows a smooth treatment of endomorphisms. In particular, if $\tilde{\perp}$ duality holds for $\mathcal{S}$ and $a \in \mathcal{S}^{\perp}$ then the endomorphism $y(a)$ associated with a 1 -cocycle satisfies $y(a)\left(\mathcal{A}\left(\mathcal{S}^{\perp}\right)\right) \subset \mathcal{A}\left(\mathcal{S}^{\perp}\right)$, because, as we have seen above, duality holds for $\mathcal{S}^{\tilde{\perp}}$ and $\mathcal{A}\left(\mathcal{S}^{\perp}\right)=\mathcal{A}\left(\mathcal{S}^{\tilde{\perp}}\right)$.

We shall be assuming $\tilde{\perp}$-duality for the elements of $\mathcal{K}$. Thus $\mathcal{K} \subset \mathcal{L}$ and $\left\{\mathcal{O}^{\perp}\right.$ : $\mathcal{O} \in \mathcal{K}\} \subset \mathcal{L}$. Thus $\mathcal{L}$ is both coinitial and cofinal in $\tilde{\mathcal{K}}$. Let us call two localized endomorphisms comparable if they are both localized in a common sieve in $\tilde{\mathcal{K}}$ and hence in some element of $\mathcal{L}$. In this case, it makes sense to talk about intertwining operators between the two localized endomorphisms. If $\rho_{i}$ is localized in $\mathcal{S}_{i}, i=1,2$, then $\rho_{1}$ and $\rho_{2}$ are comparable, if and only if $\mathcal{S}_{1} \cap \mathcal{S}_{2} \neq \emptyset$.

We turn now to the notion of left inverse. If we consider $\pi$ as a representation of the extended net $\mathcal{S} \mapsto \mathcal{A}(\mathcal{S})$, then there is an obvious modification of the notion of left inverse as we just need to replace $\mathcal{O}$ everywhere by $\mathcal{S}$. Suppose $\pi$ is localized in $\mathcal{S}$ and $\phi$ is a left inverse for $\pi$, then given $\mathcal{S}_{1} \supset \mathcal{S}$ and $\mathcal{O} \in \mathcal{S}_{1}^{\hat{\perp}}$, we remark that there is a sieve $\mathcal{S}_{2}$ with $\mathcal{O} \in \mathcal{S}_{2}$ and $\mathcal{S}_{1} \subset \mathcal{S}_{2}$. Given $A \in \mathcal{A}\left(\mathcal{S}_{1}\right)$ and $B \in \mathcal{A}(\mathcal{O})$ we have

$$
\begin{gathered}
\phi_{\mathcal{S}_{1}}(A) B=\phi_{\mathcal{S}_{2}}(A) B=\phi_{\mathcal{S}_{2}}\left(A \pi_{\mathcal{S}_{2}}(B)\right) \\
=\phi_{\mathcal{S}_{2}}\left(A \pi_{\mathcal{O}}(B)\right)=\phi_{\mathcal{S}_{2}}(A B) .
\end{gathered}
$$

Since $A$ and $B$ commute, we interchange them and reverse the steps to conclude that $\phi_{\mathcal{S}_{1}}(A)$ and $B$ commute. Recalling that $\tilde{\perp}=\hat{\perp}$ on $\tilde{\mathcal{K}}$, this proves the following result.

Lemma 3A.12 Let $\phi$ be a left inverse for a representation $\pi$ of the extended net $\mathcal{S} \mapsto \mathcal{A}(\mathcal{S})$ localized in $\mathcal{S}$. Then, if $\mathcal{S} \subset \mathcal{S}_{1}$ and $\tilde{\perp}$-duality holds for $\mathcal{S}_{1}, \phi_{\mathcal{S}_{1}} \mathcal{A}\left(\mathcal{S}_{1}\right) \subset$ $\mathcal{A}\left(\mathcal{S}_{1}\right)$.

\section{The Conformal Spin and Statistics Relation for Spacetimes With Bifurcate Killing Horizon}

In the present chapter, we shall specialize our considerations to the class of spacetimes with a bifurcate Killing horizon (bKh), whose definition we now summarize, following Kay and Wald [40]. The interested reader is strongly recommended to consult this reference for further details not spelled out here. The main purpose here is to show that, from the original theory, we can construct a family of local algebras localized on the horizon, which possesses a conformal symmetry. Therefore horizon localized superselection sectors have a conformal spin and we prove that this coincides with their statistics phase. 


\subsection{Spacetimes with bKh}

A spacetime with a bKh is a triple $\left(M, g, \tau_{t}\right)$ where $(M, g)$ is a four-dimensional, globally hyperbolic spacetime, although spacetimes with a bKh generalize to other spacetime dimensions. $\left(\tau_{t}\right)_{t \in \mathbb{R}}$ is a non-trivial one-parameter group of isometries of $(M, g)$, assumed to be $C^{\infty}$, and hence the flow of a Killing vector field $\xi$ on $M$ for the metric $g$. We often refer to $\left(\tau_{t}\right)_{t \in \mathbb{R}}$ as the Killing flow (of the spacetime with bKh). We shall assume that $(M, g)$ is orientable and that the set $\Sigma \subset M$ of fixed points of $\left(\tau_{t}\right)_{t \in \mathbb{R}}$ is a two-dimensional smooth, acausal, orientable, connected submanifold of $M$. It is worth noting that $\Sigma$, when compact, automatically lies in some Cauchy-surface, see 40 for a proof.

From this data we can construct the bKh, $\mathrm{h}$, as follows: at each point $p \in \Sigma$ we choose a pair of linearly independent, lightlike, future-oriented vectors $\chi_{A}(p), \chi_{B}(p) \in$ $T_{p} M$, normal to $\Sigma$. They are unique up to scalars and they may be chosen so that $\Sigma \ni p \mapsto \chi_{A}(p)$ and $\Sigma \ni p \mapsto \chi_{B}(p)$ are smooth vector fields along $\Sigma$ since $M$ and $\Sigma$ are orientable. Now let $\gamma_{A p}$ and $\gamma_{B p}$ be the maximal geodesics with tangents $\chi_{A}(p)$ and $\chi_{B}(p)$ at $p \in \Sigma$, respectively. Since $\left(\tau_{t}\right)$ leaves each $p \in \Sigma$ fixed, it maps each of the curves $\gamma_{A p}$ and $\gamma_{B p}$ into itself. Moreover, $\gamma_{A p}$ and $\gamma_{A p^{\prime}}$ do not intersect for $p \neq p^{\prime}$, and the same holds with $B$ in place of $A$. Now one defines sets $\mathrm{h}_{A}$ and $\mathrm{h}_{B}$ to be the lightlike hypersurfaces in $M$ formed by the $\gamma_{A p}$ and $\gamma_{B p}$, respectively, as $p$ ranges over $\Sigma$. Then $\mathrm{h}:=\mathrm{h}_{A} \cup \mathrm{h}_{B}$ is the $\mathrm{bKh}$, and one distinguishes the following subsets:

$$
\begin{array}{ll}
\mathrm{h}_{A}^{R}:=\left(\mathrm{h}_{A} \backslash \Sigma\right) \cap J^{+}(\Sigma), & \mathrm{h}_{A}^{L}:=\left(\mathrm{h}_{A} \backslash \Sigma\right) \cap J^{-}(\Sigma), \\
\mathrm{h}_{B}^{R}:=\left(\mathrm{h}_{B} \backslash \Sigma\right) \cap J^{-}(\Sigma), & \mathrm{h}_{B}^{L}:=\left(\mathrm{h}_{B} \backslash \Sigma\right) \cap J^{+}(\Sigma) .
\end{array}
$$

The Killing vector field $\xi$ is conventionally assumed to be future oriented on $\mathrm{h}_{A}^{R}$. The bKh divides the spacetime $M$ locally into four disjoint parts, $F:=J^{+}(\Sigma)$, $P:=J^{-}(\Sigma), R:=\left(J^{-}\left(\mathrm{h}_{A}^{R}\right) \backslash \mathrm{h}_{A}^{R}\right) \cap\left(J^{+}\left(\mathrm{h}_{B}^{R}\right) \backslash \mathrm{h}_{B}^{R}\right)$ and $L:=\left(J^{-}\left(\mathrm{h}_{B}^{L}\right) \backslash \mathrm{h}_{B}^{L}\right) \cap\left(J^{+}\left(\mathrm{h}_{A}^{L}\right) \backslash \mathrm{h}_{A}^{L}\right)$, the future, past, right and left parts of the bKh, respectively.

To give a rather simple illustration, consider $(M, g)$ as Minkowski spacetime (of dimension 4). Then choose an inertial coordinate system and define $\Sigma$ as the twodimensional hyperplane $\left\{\left(x^{0}, x^{1}, x^{2}, x^{3}\right) \in \mathbb{R}^{4}: x^{0}=x^{1}=0\right\}$. There is a smooth, one-parameter group $\tau_{t}=\Lambda_{t}, t \in \mathbb{R}$, of pure Lorentz transformations leaving $\Sigma$ fixed; they are defined by

$$
\Lambda_{t}\left(x_{0}, x_{1}, x_{2}, x_{3}\right):=\left(\cosh (t) x_{0}+\sinh (t) x_{1}, \sinh (t) x_{0}+\cosh (t) x_{1}, x_{2}, x_{3}\right) .
$$

Then $\mathrm{h}=\mathrm{h}_{A} \cup \mathrm{h}_{B}$ is a bKh, where $\mathrm{h}_{A}=\left\{\left(u, u, x_{2}, x_{3}\right): u \in \mathbb{R},\left(x_{2}, x_{3}\right) \in \mathbb{R}^{2}\right\}$ and $\mathrm{h}_{B}=\left\{\left(v,-v, x_{2}, x_{3}\right): v \in \mathbb{R},\left(x_{2}, x_{3}\right) \in \mathbb{R}^{2}\right\}$. Here, the regions $R$ and $L$ correspond to the usual "right wedge" and "left wedge" regions in Minkowski spacetime. Other important examples of spacetimes with a bKh include e.g. deSitter and SchwarzschildKruskal spacetimes, as well as the Schwarzschild-deSitter spacetimes and (certain regions of the) Kerr-Newman spacetimes. (The latter have at least two bKhs with different surfaces gravities, see below. This leads [40] to conclude that there are no regular, Killing-flow invariant states of the free scalar field on such spacetimes.)

Let us now look at how the Killing flow acts on the bKh in greater detail. Each of the geodesic generators $\gamma_{A p}$ of the $\mathrm{h}_{A}$-part of the $\mathrm{bKh}$ is defined on some interval 
$I_{p}$. We may choose an affine parametrization of $\gamma_{A p}$, with affine parameter $U$, such that $\gamma_{A p}(U=0)=p$ and $\left.\frac{d}{d U} \gamma_{A p}\right|_{U=0}=\chi_{A}(p)$ for all $p \in \Sigma$. This parametrizes all the geodesics and, since the vector field $\chi_{A}(p)$ depends smoothly on $p \in \Sigma$, by assumption, the affine parametrization of the curves $\gamma_{A p}$ depends smoothly on $p \in \Sigma$. Since $\gamma_{A p}$ is left invariant under the Killing flow, $I_{p}$ must be invariant under a (non-trivial) smooth representation of the additive group $\mathbb{R}$ (with 0 as the only fixed point), and thus $I_{p}=\mathbb{R}$. A similar result holds for the domains of the geodesic generators $\gamma_{B p}$ of $\mathrm{h}_{B}$. Therefore, each point $q \in \mathrm{h}_{A}$ is uniquely determined by the pair $(U, p)$, where $q=\gamma_{A p}(U)$. Hence we have a diffeomorphism $\psi_{A}: \mathrm{h}_{A} \rightarrow \mathbb{R} \times \Sigma$ assigning to $q \in \mathrm{h}_{A}$ the pair $(U, p) \in \mathbb{R} \times \Sigma$ with $q=\gamma_{A p}(U)$. I As explained below, certain choices of $\chi_{A}$ and $\chi_{B}$ turn out to be particularly useful for our purposes and lead to the following relation (cf. [40], see also [57]):

$$
\tau_{t} \circ \psi_{A}{ }^{-1}(U, p)=\psi_{A}{ }^{-1}\left(\mathrm{e}^{\kappa t} U, p\right), \quad t, U \in \mathbb{R}, p \in \Sigma,
$$

where the number $\kappa>0$, called the surface gravity, is an invariant of the bKh under consideration. (For the Schwarzschild-Kruskal spacetime of a black hole with mass $m_{\mathrm{bh}}>0, \kappa$ is proportional to $m_{\mathrm{bh}}$. The reader is referred to 40, 61, for more information about the notion of surface gravity.) Constructing a diffeomorphism $\psi_{B}: \mathrm{h}_{B} \rightarrow \mathbb{R} \times \Sigma$, similarly, where $\psi_{B}(q)=(V, q)$ iff $q=\gamma_{B p}(V)$, the affine geodesic parameter being now denoted by $V$, one can show that

$$
\tau_{t} \circ \psi_{B}{ }^{-1}(V, p)=\psi_{B}{ }^{-1}\left(\mathrm{e}^{-\kappa t} V, p\right), \quad t, V \in \mathbb{R}, p \in \Sigma,
$$

with the same $\kappa>0$ as in the previous equation.

There are a few other geometric actions on $\mathrm{h}_{A}$ and $\mathrm{h}_{B}$, induced by identifying these parts of the bKh with $\mathbb{R} \times \Sigma$ via the maps $\psi_{A}$ and $\psi_{B}$. First, there are the affine translations

$$
\begin{aligned}
\ell_{a} \circ \psi_{A}{ }^{-1}(U, p) & :=\psi_{A}{ }^{-1}(U+a, p), \\
\ell_{a} \circ \psi_{B}{ }^{-1}(V, p) & :=\psi_{B}{ }^{-1}(V+a, p), \quad a, U, V \in \mathbb{R}, p \in \Sigma .
\end{aligned}
$$

In contrast to the dilations on $\mathrm{h}_{A}$ and $\mathrm{h}_{B}$, induced by restricting the Killing flow to the $\mathrm{bKh}$, the translations will not, in general, extend to isometries of the full spacetime. Another action is the (affine) reflection, 凹

$$
\begin{aligned}
& \iota \circ \psi_{A}{ }^{-1}(U, p):=\psi_{A}^{-1}(-U, p), \\
& \iota \circ \psi_{B}{ }^{-1}(V, p):=\psi_{B}{ }^{-1}(-V, p), \quad U, V \in \mathbb{R}, p \in \Sigma .
\end{aligned}
$$

Again, $\iota$ need not extend to an isometry of the full spacetime to the bKh. However, Kay and Wald 40] have shown that, if the spacetime with bKh is analytic, there is

\footnotetext{
${ }^{6}$ Notice that $\psi_{A}$ depends on the choice of the vector field $\Sigma \ni p \mapsto \chi_{A}(p)$ along $\Sigma$. It may be rescaled at each point: $\tilde{\chi}_{A}(p)=\phi(p) \chi_{A}(p)$, with $\phi: \Sigma \rightarrow \mathbb{R}$ a smooth, strictly positive function, would serve just as well when constructing $\mathrm{h}_{A}$. A similar remark applies to the $\mathrm{h}_{B}$-horizon.

${ }^{7}$ The definitions of $\ell_{a}$ and $\iota$ involve $\psi_{A}$ (or $\psi_{B}$ ) so these quantities, cf. the previous footnote, depend on the scaling freedom when choosing $\psi_{A}$ (or $\psi_{B}$ ).
} 
a neighbourhood $N$ of $\mathrm{h}$ and an orientation and chronology-reversing isometry $\mathbf{j}$ of $N$ ("horizon reflection") commuting with the action of $\left(\tau_{t}\right)$ which reflects the affine parameter of geodesics passing orthogonally through $\Sigma$.

In the next step, we shall specify some families of regions analogous in some respects to the "shifted wedges" in Minkowski spacetime. With their help, we can then formulate a version of geometric modular action for quantum field theories on spacetimes with a bKh in the operator-algebraic framework. To begin with, we note (cf. [40]) that the parts $F, P, R$ and $L$ of a spacetime with bKh (see above) satisfy

$$
\begin{array}{ll}
F \cap P=\Sigma, & F \cap R=\emptyset, \quad P \cap R=\emptyset, \\
F \cap L=\emptyset, & P \cap L=\emptyset .
\end{array}
$$

Thus, as we have already seen from the example above, $R$ and $L$ may be viewed as playing the role of the right and left wedge regions in Minkowski spacetime. If $\tilde{M}:=F \cup P \cup L \cup R$, then $\tilde{M}, L$ and $R$, with the appropriate restrictions of $g$ as Lorentzian metric, are globally hyperbolic spacetimes. It may, however, happen that $\tilde{M} \neq M$, see 40 for examples. As we shall later assume that $M=\tilde{M}$, this possibility need not concern us. One can see from (2.3) that the regions $F, P, R$ and $L$ are invariant under the Killing flow $\left(\tau_{t}\right)$. This implies that $\tilde{M}$ is also invariant under $\left(\tau_{t}\right)$. For open intervals $(a, b)$ with $a<b$ and $a, b \in \mathbb{R} \cup\{ \pm \infty\}$, we now define

$$
\mathrm{h}_{A}(a, b):=\left\{\psi_{A}{ }^{-1}(U, p): a<U<b, p \in \Sigma\right\}
$$

with an analogous definition of $\mathrm{h}_{B}(a, b)$. Notice that with this notation,

$$
\mathrm{h}_{A}^{R}=\mathrm{h}_{A}(0, \infty), \quad \mathrm{h}_{A}^{L}=\mathrm{h}_{A}(-\infty, 0) .
$$

The "shifted right wedge" can then be defined as

$$
R_{a}:=R \backslash \mathrm{cl} J^{-}\left(\mathrm{h}_{A}(-\infty, a)\right)
$$

for $a>0$, where cl means "closure".

\section{Lemma 4.1.}

$$
\tau_{t}\left(R_{a}\right)=R_{\mathrm{e}^{\kappa t} \cdot a} \text { for all } t \in \mathbb{R}, a \geq 0 .
$$

Proof. Since $\left(\tau_{t}\right)$ is a group of isometries leaving $R$ invariant,

$$
\begin{aligned}
\tau_{t}\left(R_{a}\right) & =\tau_{t}\left(R \backslash \mathrm{cl} J^{-}\left(\mathrm{h}_{A}(-\infty, a)\right)\right) \\
& =\tau_{t}(R) \backslash \tau_{t}\left(\operatorname{cl} J^{-}\left(\mathrm{h}_{A}(-\infty, a)\right)\right) \\
& =R \backslash \operatorname{cl} J^{-}\left(\mathrm{h}_{a}\left(-\infty, \mathrm{e}^{\kappa t} \cdot a\right)\right) \\
& =R_{\mathrm{e}^{\kappa t} \cdot a} .
\end{aligned}
$$


Similarly, setting

$$
L_{-a}:=L \backslash \operatorname{cl} J^{+}\left(\mathrm{h}_{A}(-\infty,-a)\right)
$$

for $a>0(!)$, we find as before that

$$
\tau_{t}\left(L_{-a}\right)=L_{-\mathrm{e}^{-\kappa t} \cdot a}, \quad t \in \mathbb{R}, a>0 .
$$

In this section, a non-void open $\mathcal{O} \subset M$ is called a diamond if it is of the form $\mathcal{O}=\operatorname{int} D(G)$ where $G$ is an open subset of a Cauchy surface $C$ (not necessarily acausal) such that $\partial G$ is continuous and $\mathcal{O}^{\perp}$ non-void; moreover $\mathcal{O}$ or $\mathcal{O}^{\perp}$ is required to be connected.

Below we study nets of von Neumann algebras indexed by the diamond regions in a given spacetime with bKh. Hence we would like the regions $R_{a}$ and $L_{-a}$ to be diamonds. Our task is thus to verify this if $\chi_{A}$ and $\chi_{B}$ are chosen suitably. By assumption, there is an acausal Cauchy surface $C$ passing through $\Sigma$. Let $C_{1}$ be another acausal Cauchy surface lying strictly in the future of $C$, i.e. $C_{1} \subset \operatorname{int} J^{+}(C)=$ $J^{+}(C) \backslash C$. Then we suppose that $\chi_{A}$ has been chosen such that each point $q \in \mathrm{h}_{A} \cap C_{1}$ has affine parameter $U=1$, which means that $q=\psi^{-1}(1, p)$ for some $p \in \Sigma$. Clearly such a choice is always possible (it amounts to a suitable choice of the smooth rescaling function $\phi: \Sigma \rightarrow \mathbb{R}$ ). Under the Killing flow $\tau_{t}$ we get a family $C_{\mathrm{e}^{\kappa t}}:=\tau_{t}\left(C_{1}\right), t \in \mathbb{R}$, of acausal Cauchy surfaces (not necessarily forming a foliation) having the property that each $q \in \mathrm{h}_{A} \cap C_{\mathrm{e}^{\kappa t}}$ is represented as $q=\psi_{A}^{-1}\left(\mathrm{e}^{\kappa t}, p\right)$ with suitable $p \in \Sigma$. Obviously, a similar construction can be carried out with a Cauchy surface $C_{-1}$ lying strictly in the past of $C$ and leads to family of acausal Cauchy surfaces $C_{-\mathrm{e}^{\kappa t}}=\tau_{t}\left(C_{-1}\right)$. (Moreover, similar constructions can be made for $\chi_{B}, \mathrm{~h}_{B}$. ) As we first chose $C_{1}$ and then adjusted $\chi_{A}$ to give all points of $C_{1} \cap \mathrm{h}_{A}$ affine parameter $U=1$ it is not obvious that we can choose $C_{-1}$ to give all points of $C_{-1} \cap \mathrm{h}_{A}$ affine parameter $U=-1$. It would suffice if there were a global isometry of $\mathrm{M}$ acting as a horizon-reflection symmetry $\mathbf{j}$ since then one may simply choose $C_{-1}=\mathbf{j}\left(C_{1}\right)$. The existence of such an isometry will be required later, but not for the next lemma, where an arbitrary pair of Cauchy surfaces $C_{1}$ and $C_{-1}$ with the indicated properties is assumed given, and the corresponding vector fields $\chi_{A}$ and $\chi_{A}^{(-)}$assumed chosen so that each point on $C_{1} \cap \mathrm{h}_{A}$ has affine parameter $U=1$ with respect to $\chi_{A}$ and each point on $C_{-1} \cap \mathrm{h}_{A}$ affine parameter $U=-1$ with respect to $\chi_{A}^{(-)}$.

Lemma 4.2. If $M=\tilde{M}$, then $R^{\perp}=L, L^{\perp}=R$ and $R, L$ and $R_{a}, L_{-a}, a>0$, are diamonds.

Proof. By assumption, we have $M=F \cup P \cup R \cup L$, and $F \cup P=J(\Sigma)$. Since $\Sigma$ is part of a Cauchy surface, it follows that $\Sigma^{\perp}=\operatorname{int} D(C \backslash \Sigma)$. Hence $R \cup L=\operatorname{int} D(C \backslash \Sigma)$. Now define $C_{R}:=C \cap R, C_{L}:=C \cap L$. Then $C_{R} \cap C_{L}=\emptyset$ since $L \cap R=\emptyset$ (see 401), and $C_{L} \cup C_{R}=C \backslash \Sigma$. Therefore we obtain int $D(C \backslash \Sigma)=\operatorname{int} D\left(C_{R} \cup C_{L}\right)=$ $\operatorname{int} D\left(C_{L}\right) \cup \operatorname{int} D\left(C_{R}\right)$ where the last equality is a consequence of the fact that $C_{L}$ and $C_{R}$ are disjoint open subsets of a Cauchy surface. The boundary of $C_{L}$ and $C_{R}$ is in both cases the smooth manifold $\Sigma$. Hence $L=\operatorname{int} D\left(C_{L}\right)$ and $R=\operatorname{int} D\left(C_{R}\right)$ 
are diamonds, and since $C_{L}$ and $C_{R}$ are disjoint and their union yields $C$ up to the common boundary $\Sigma$ of $C_{L}$ and $C_{R}$, this entails $R^{\perp}=L$ and $L^{\perp}=R$.

Now we define the following sets: $\Sigma_{a}:=C_{a} \cap \mathrm{h}_{A}, C_{a R}:=C_{a} \cap R, C_{a L}:=C_{a} \cap L$, $C_{a F}=C_{a} \cap F$. One can see that $C_{a} \cap P=\emptyset$, for there would otherwise be causal curves joining pairs of points on $C_{a}$ and this is excluded. It follows that $C_{a}=$ $C_{a L} \cup C_{a R} \cup C_{a F}$ is the union of three disjoint parts, and int $D\left(C_{a R}\right)=\left(C_{a F} \cup\right.$ $\left.C_{a L}\right)^{\perp}$. The common boundary of $C_{a R}$ and $C_{a L} \cup C_{a F}$ is the smooth manifold $\Sigma_{a}$, implying that int $D\left(C_{a R}\right)$ is a diamond. Moreover, it is obvious that $\mathrm{h}_{A}(a, \infty) \subset$ $J^{+}\left(\Sigma_{a}\right), \mathrm{h}_{A}(-\infty, a) \subset J^{-}\left(\Sigma_{a}\right)$, and by standard arguments it follows that $J^{+}\left(\Sigma_{a}\right)=$ $\operatorname{cl} J^{+}\left(\mathrm{h}_{A}(a, \infty)\right)$ and $J^{-}\left(\Sigma_{a}\right)=\mathrm{cl} J^{-}\left(\mathrm{h}_{A}(-\infty, a)\right)$. Let us check that $R_{a}=\operatorname{int} D\left(C_{a R}\right)$. First we notice that int $D\left(C_{a R}\right) \subset R$ is fairly obvious ( $R$ is causally closed, i.e. $R^{\perp \perp}=R$, and $C_{a R}$ is an acausal hypersurface in $\left.R\right)$, and so is $\operatorname{int} D\left(C_{a R}\right)=\left(C_{a F} \cup\right.$ $\left.C_{a L}\right)^{\perp} \subset \Sigma_{a}^{\perp}=M \backslash J\left(\Sigma_{a}\right)$, implying int $D\left(C_{a R}\right) \subset R_{a}$. To show the reverse inclusion it is sufficient to prove that $R_{a} \cap \operatorname{cl} J\left(C_{a F} \cup C_{a L}\right)=\emptyset$. We have $\operatorname{cl} J\left(C_{a F} \cup C_{a L}\right)=$ $\operatorname{cl} J\left(C_{a F}\right) \cup \operatorname{cl} J\left(C_{a L}\right)$ and $C_{a L} \subset L$ and $R=L^{\perp}$ imply that $R \cap \operatorname{cl} J\left(C_{a L}\right)=\emptyset$. Now consider an arbitrary past-directed causal curve $\gamma$ starting at some point on $C_{a F}$. For $\gamma$ to meet $R_{a}$, it must intersect $\mathrm{h}_{A}$. However, any intersection of $\gamma$ with $\mathrm{h}_{A}$ must be contained in $\mathrm{h}_{A}(-\infty, a]$ since $\gamma$ is past-directed and we have seen that $\mathrm{h}_{A}(a, \infty) \subset J^{+}\left(\Sigma_{a}\right) \subset J^{+}\left(C_{a F}\right)$. Thus, since only the part of $\gamma$ lying in the causal past of its intersection with $\mathrm{h}_{A}$ can enter $R, \gamma$ never meets $R_{a}=R \backslash c l J^{-}\left(\mathrm{h}_{A}(-\infty, a)\right)$, showing that $\mathrm{cl} J\left(C_{a F}\right) \cap R_{a}=\emptyset$. Therefore $R_{a}=\operatorname{int} D\left(C_{a R}\right)$ is a diamond. An analogous argument works for $L_{-a}$.

\subsection{Conformal Spin-Statistics Relation}

Our aim in this subsection will be to show that the net $\mathcal{O} \mapsto \mathcal{A}(\mathcal{O})$ on a spacetime with bKh induces a net of von Neumann algebras $(a, b) \mapsto \mathcal{C}(a, b)$, indexed by the open intervals $(a, b)$ of the real line and allowing an extension to a conformally covariant theory on the circle $S^{1}$. Moreover, we shall see that this net is to be viewed as containing precisely the observables localized arbitrarily closely to the $\mathrm{h}_{A}$-horizon. (A similar construction works for the $\mathrm{h}_{B}$-horizon). The variant of Wiesbrock's results on modular inclusion [64] which is needed to show this may be familiar to experts, but for the reader's convenience we present the arguments in an appendix to this chapter (Sec. 4.3).

Earlier results [32, 33] on the spin-statistics connection for conformally covariant theories on $S^{1}$ then apply, yielding a conformal spin-statistics theorem for the subnets of the initial theory consisting of observables concentrated on the parts $h_{A}$ and $h_{B}$ of the horizon.

We begin with a spacetime with a bKh, $\left(M, g, \tau_{t}, \Sigma, \mathrm{h}\right)$, where we assume henceforth that $M=\tilde{M}$ (cf. Sec. 4.1). Furthermore, we assume given a net $\mathcal{K} \ni \mathcal{O} \mapsto \mathcal{A}(\mathcal{O})$ assigning to each member $\mathcal{O}$ in the collection $\mathcal{K}$ of regions in $M$ a von Neumann algebra $\mathcal{A}(\mathcal{O})$ on a Hilbert space $\mathcal{H}_{\mathcal{A}}$. For convenience, we shall work not with $\mathcal{K}$, the collection of regular diamonds ordered under inclusion, but extend the domain of our observable net $\mathcal{A}$ in the canonical way to include a larger collection $\mathcal{L}$ of open subsets of our spacetime. As discussed in the appendix to Sec. 3, this choice does not 
change the superselection structure in that each representation satisfying the selection criterion based on $\mathcal{K}$ extends uniquely to a representation satisfying the selection criterion based on $\mathcal{L}$, the intertwining operators thereby remaining unchanged. Again as discussed in the Appendix to Sec. 3, the formalism changes only in so far as the localized endomorphisms are now defined on larger algebras and this proves to be an advantage. We choose $\mathcal{L}$ to be the set of non-empty causally closed subsets $\mathcal{S}$ of $M$ with non-empty causal complements such that for the given net $\mathcal{A} \tilde{\perp}$-duality holds either for $\mathcal{S}$ or for $\mathcal{S}^{\perp}$. By virtue of Lemma 3A.4, this is the same as the partially ordered set $\mathcal{L}$ defined in the Appendix to Sec. 3 in terms of sieves. We recall, too, that if $\mathcal{A}$ is additive, or even inner regular, as a net over $\mathcal{K}$, then $\tilde{\perp}$-duality coincides with $\perp$-duality.

Indeed, even though we assume duality for all diamonds, such assumption is actually used only for two kinds of regions, the translated wedges $L_{a}$ and $R_{a}$, and some tubular neighborhoods of the horizon intervals $\mathrm{h}_{A}(a, b)$ or $\mathrm{h}_{B}(a, b)$, which are in turn tubular neighborhoods in $\mathrm{h}_{A}$ or $\mathrm{h}_{B}$ of a suitable translation of $\Sigma$. We observe that the obstructions to duality are usually homological in nature, and that is why duality is generally assumed to hold for regular diamonds. On the other hand the surface $\Sigma$, even though not necessarily homologically trivial, is often relatively trivial, meaning that $k$-cycles in $\Sigma$ which are trivial in $M$ are trivial in $\Sigma$ too.

In the following we shall consider the subnet of $\mathcal{O} \mapsto \mathcal{A}(\mathcal{O})$ generated by the observables located arbitrarily closely to the (half) horizon $\mathrm{h}_{A}$. Let us adopt the setting of Lemma 4.2 and start with a given acausal Cauchy surface $C$ containing $\Sigma$ and choose an acausal Cauchy surface $C_{1}$ lying strictly in the future of $C$ and the vector field $\chi_{A}$ so that each point on $C_{1} \cap \mathrm{h}_{A}$ has affine parameter $U=1$. Then we define for $0<a<b<\infty$,

$$
\mathcal{B}_{A}^{R}(a, b):=\bigcap_{\mathcal{O}}\left\{\mathcal{A}(\mathcal{O}): \mathcal{O} \supset \overline{\mathrm{h}_{A}(a, b)}\right\}
$$

where the intersection is taken over diamonds $\mathcal{O}$. Likewise, one may also assume that another acausal Cauchy surface $C_{-1}$, lying strictly in the past of $C$, has been selected and that another (possibly identical) copy $\chi_{A}^{(-)}$of $\chi_{A}$ has been chosen to give each point of $C_{-1} \cap \mathrm{h}_{A}$ an affine parameter $U=-1$. Correspondingly, we set for $-\infty<-b<-a<0$;

$$
\mathcal{B}_{A}^{L}(-b,-a):=\bigcap_{\mathcal{O}}\left\{\mathcal{A}(\mathcal{O}): \mathcal{O} \supset \overline{\mathrm{h}_{A}(-b,-a)}\right\} .
$$

Finally, with these assumptions, one may also define

$$
\mathcal{B}_{A}\left(a^{\prime}, b^{\prime}\right):=\bigcap_{\mathcal{O}}\left\{\mathcal{A}(\mathcal{O}): \mathcal{O} \supset \overline{\mathrm{h}_{A}\left(a^{\prime}, b^{\prime}\right)}\right\}
$$

for $-\infty<a^{\prime}<b^{\prime}<\infty$. Substituting $B$ for $A$ in the above, algebras $\mathcal{B}_{B}^{R / L}(a, b)$, $\mathcal{B}_{B}\left(a^{\prime}, b^{\prime}\right)$ can be defined and all results formulated in the sequel for the algebras $\mathcal{B}_{A}$ hold with obvious modifications for the algebras $\mathcal{B}_{B}$ too. 
Lemma 4.3. Suppose that the net $\mathcal{O} \mapsto \mathcal{A}(\mathcal{O})$ satisfies the following assumptions:

- (I) Irreducibility: $\vee_{\mathcal{O} \in \mathcal{K}} \mathcal{A}(\mathcal{O})=B(\mathcal{H})$.

- (II) Additivity: $\mathcal{O} \subset \cup_{i \in I} \mathcal{O}_{i}, \quad \mathcal{O}_{i}, \mathcal{O} \in \mathcal{K} \quad \Rightarrow \quad \mathcal{A}(\mathcal{O}) \subset \vee_{i \in I} \mathcal{A}\left(\mathcal{O}_{i}\right)$.

- (III) Haag duality: $\mathcal{A}\left(\mathcal{O}^{\perp}\right)=\mathcal{A}(\mathcal{O})^{\prime}, \quad \mathcal{O} \in \mathcal{K} \quad$ (implying locality).

Then

$$
\begin{aligned}
\mathcal{B}_{A}^{R}(a, b) & =\mathcal{A}\left(R_{a}\right) \cap \mathcal{A}\left(R_{b}\right)^{\prime} \\
\mathcal{B}_{A}^{L}(-b,-a) & =\mathcal{A}\left(L_{-a}\right) \cap \mathcal{A}\left(L_{-b}\right)^{\prime} \\
\mathcal{B}_{A}\left(-a^{\prime}, b\right) & =\mathcal{A}\left(L_{-a^{\prime}}\right)^{\prime} \cap \mathcal{A}\left(R_{b}\right)^{\prime}
\end{aligned}
$$

for all $0<a<b<\infty,-a^{\prime}<0$.

Proof. We shall only give the proof of the first equality, since the remaining cases are completely analogous, requiring some largely obvious notational changes.

We recall that $C_{a}=\tau_{\ln a / \kappa}\left(C_{1}\right)$ for any $a>0$, and also the notation $\Sigma_{a}=C_{a} \cap \mathrm{h}_{A}$, $C_{a R}=C_{a} \cap R, C_{a F}=C_{a} \cap F$ and $C_{a L}=C_{a} \cap L$ used in the proof of Lemma 4.2. Then we define the subsets $\widetilde{L}_{a}:=\left(R_{a}\right)^{\perp}=\operatorname{int} D\left(C_{a L} \cup C_{a F}\right), F_{a}:=J^{+}\left(\Sigma_{a}\right)$ and $P_{a}:=J^{-}\left(\Sigma_{a}\right)$, and analogous sets with $a$ replaced by $b$. Next, we define $C^{\vee}:=$ $C_{a L} \cup C_{a F} \cup \overline{\mathrm{h}_{A}(a, b)} \cup C_{a R}$, and aim at demonstrating that this set is a Cauchy surface. It is fairly obvious that $C^{\vee}$ is achronal, i.e. $C^{\vee} \cap i n t ~ J^{ \pm}\left(C^{\vee}\right)=\emptyset$. It is also not difficult to check that $M=\widetilde{L}_{a} \cup R_{b} \cup F_{a} \cup P_{b}$ where the sets forming the union are pairwise disjoint except for the intersection $F_{a} \cap P_{b}=\overline{\mathrm{h}_{A}(a, b)}$. Now let $\gamma$ be an arbitrary endpointless causal curve in $M$. If $\gamma$ enters $\widetilde{L}_{a}$ or $R_{b}$, it must intersect $\overline{C_{a L} \cup C_{a F}}$ or $\overline{C_{b R}}$, hence $C^{\vee}$. Suppose that $\gamma$ enters $F_{a}$. Since $F_{a}$ is past-compact, $\gamma$ must intersect one of the regions $R_{b}, \widetilde{L}_{a}$ or $P_{b}$, as $\gamma$ would otherwise have a past-endpoint. On the other hand, a causal curve without endpoint intersecting $F_{a}$ can only meet $P_{b}$ if it intersects $\overline{\mathrm{h}_{A}(a, b)}$, too. Hence, if $\gamma$ enters $F_{a}$, it must also intersect $C^{\vee}$. Using the same argument with obvious modifications for the case that $\gamma$ enters $P_{b}$, one arrives at the same conclusion. This shows that every causal curve without endpoints in $M$ intersects $C^{\vee}$, implying $M=D\left(C^{\vee}\right)$, and therefore $C^{\vee}$ is a Cauchy surface.

Now we note that int $D(U) \supset \overline{\mathrm{h}_{A}(a, b)}$ for each open neighbourhood $U$ of $\overline{\mathrm{h}_{A}(a, b)}$ in $C^{\vee}$ since $J\left(\overline{\mathrm{h}_{A}(a, b)}\right)=P_{b} \cup F_{a}$ has empty intersection with $\operatorname{cl}\left(C^{\vee} \backslash U\right)$. Thus $\overline{\mathrm{h}_{A}(a, b)}$ is an intersection of diamonds. Moreover, whenever $\mathcal{O} \supset \overline{\mathrm{h}_{A}(a, b)}$ is any diamond, it is obvious that we can find some open subset $U$ of $C^{\vee}$ with piecewise smooth boundary $\overline{\mathrm{h}_{A}(a, b)} \subset U \subset \mathcal{O} \cap C^{\vee}$, implying $\overline{\mathrm{h}_{A}(a, b)} \subset$ int $D(U) \subset \mathcal{O}$. Hence, to establish the lemma, it suffices to consider diamonds of the form $\mathcal{O}=\operatorname{int} D(U)$. Obviously, the causal complement $\mathcal{O}^{\perp}$ of each such $\mathcal{O}$ may be written as $\mathcal{O}^{\perp}=\mathcal{O}_{R}^{\perp} \cup \mathcal{O}_{L}^{\perp}$ where $\mathcal{O}_{R}^{\perp}=\mathcal{O}^{\perp} \cap R_{a}=\operatorname{int} D\left(C_{a R} \backslash \bar{U}\right)$ and $\mathcal{O}_{L}^{\perp}=\mathcal{O}^{\perp} \cap \widetilde{L}_{a}=\operatorname{int} D\left(\left(C_{a L} \cup C_{a F}\right) \backslash \bar{U}\right)$ are both diamonds. Notice that the union of $\mathcal{O}_{L}^{\perp}$ and $\mathcal{O}_{R}^{\perp}$ over all $\mathcal{O}=\operatorname{int} D(U)$ yield $\widetilde{L}_{a}$ and 
$R_{b}$, respectively. Consequently we have

$$
\begin{aligned}
\bigcap_{\mathcal{O}} \mathcal{A}(\mathcal{O}) & =\left(\bigvee_{\mathcal{O}} \mathcal{A}(\mathcal{O})^{\prime}\right)^{\prime}=\left(\bigvee_{\mathcal{O}} \mathcal{A}\left(\mathcal{O}^{\perp}\right)\right)^{\prime} \\
& =\left(\bigvee_{\mathcal{O}} \mathcal{A}\left(\mathcal{O}_{L}^{\perp} \cup \mathcal{O}_{R}^{\perp}\right)\right)^{\prime}=\left(\bigvee_{\mathcal{O}} \mathcal{A}\left(\mathcal{O}_{L}^{\perp}\right) \vee \mathcal{A}\left(\mathcal{O}_{R}^{\perp}\right)\right)^{\prime} \\
& =\left(\mathcal{A}\left(\widetilde{L}_{a}\right) \vee \mathcal{A}\left(R_{b}\right)\right)^{\prime}=\left(\mathcal{A}\left(R_{a}\right)^{\prime} \vee \mathcal{A}\left(R_{b}\right)\right)^{\prime} \\
& =\mathcal{A}\left(R_{a}\right) \cap \mathcal{A}\left(R_{b}\right)^{\prime},
\end{aligned}
$$

where the second equality follows from Haag duality, the third has been justified above, the fourth and fifth equalities use additivity and the last but one again follows from Haag duality.

The formulation of the subsequent result necessitates introducing further assumptions and related notation.

We shall write $\mathcal{B}_{A}^{R}(a, \infty):=\bigvee_{b>a} \mathcal{B}_{A}^{R}(a, b)$, and define the other horizon-algebras associated with unbounded intervals in a similar manner by additivity. Let $\Omega \in$ $\underline{\mathcal{H}}$ be a unit vector vector, then we denote by $\mathcal{H}_{A}^{R}(\Omega):=\overline{\mathcal{B}_{A}^{R}(0, \infty) \Omega}, \mathcal{H}_{A}^{L}(\Omega):=$ $\overline{\mathcal{B}_{A}^{L}(-\infty, 0) \Omega}$ and $\mathcal{H}_{A}(\Omega):=\overline{\mathcal{B}_{A}(-\infty, \infty) \Omega}$ the Hilbert subspaces generated by applying the various algebras of observables concentrated on the $\mathrm{h}_{A}$-horizon on that vector. We say that $\left(\mathcal{B}^{R}(0, \infty), \Omega\right)$ is a standard pair if $\Omega$ is separating for $\mathcal{B}^{R}(0, \infty)$. It is by definition cyclic with respect to the Hilbert subspace $\mathcal{H}_{A}^{R}(\Omega)$. The modular objects (with respect to $\mathcal{H}_{A}^{R}(\Omega)$ ) of such a standard pair will be denoted by $J_{R, \Omega}$, $\Delta_{R, \Omega}$. The like objects for $L$ in place of $R$ are defined similarly.

In the following, we shall focus attention on the next two assumptions:

(IV) Geometric modular group on the horizon: There is a unit vector $\Omega \in \mathcal{H}$ so that

(i) $\quad\left(\mathcal{B}_{A}^{R}(0, \infty), \Omega\right)$ is a standard pair, and

$$
\Delta_{R, \Omega}^{i t} \mathcal{B}_{A}^{R}(a, \infty) \Delta_{R, \Omega}^{-i t}=\mathcal{B}_{A}^{R}\left(\mathrm{e}^{-2 \pi t / \kappa} a, \infty\right),
$$

(ii) $\quad\left(\mathcal{B}_{A}^{L}(0, \infty), \Omega\right)$ is a standard pair, and

$$
\Delta_{L, \Omega}^{i t} \mathcal{B}_{A}^{L}(-\infty,-a) \Delta_{L, \Omega}^{-i t}=\mathcal{B}_{A}^{L}\left(-\infty,-\mathrm{e}^{2 \pi t / \kappa} a\right),
$$

for all $a>0, t \in \mathbb{R}$, where $\kappa>0$ is the surface gravity of the bKh.

(V) Geometric modular conjugation on the horizon: For the $\Omega$ as in (IV), we have $\mathcal{H}_{A}(\Omega)=\mathcal{H}_{A}^{R}(\Omega)=\mathcal{H}_{A}^{L}(\Omega)$ and moreover

$$
J_{R, \Omega} \mathcal{B}_{A}^{R}(a, \infty) J_{R, \Omega}=\mathcal{B}_{A}^{L}(-\infty,-a), \quad a \geq 0 .
$$

Let us now assume that the net $\mathcal{O} \mapsto \mathcal{A}(\mathcal{O})$ satisfies assumptions (I-IV). Thus we see that $\left(\mathcal{B}_{A}^{R}(1, \infty) \subset \mathcal{B}_{A}^{R}(0, \infty), \Omega\right)$ is a thsm inclusion and $\left(\mathcal{B}_{A}^{L}(-\infty,-1) \subset\right.$ $\left.\mathcal{B}_{A}^{L}(-\infty, 0), \Omega\right)$ is a - hsm inclusion. Then the results of [64, [1] yield two continuous unitary groups $U^{R / L}(a), a \in \mathbb{R}$, having positive/negative spectrum and satisfying the 
following relations for $a>0$ :

$$
\begin{array}{rlrl}
\Delta_{R}^{-i t} U^{R}(a) \Delta_{R}^{i t} & =U^{R}\left(\mathrm{e}^{2 \pi t} a\right), & \Delta_{L}^{i t} U^{L}(a) \Delta_{L}^{-i t}=U^{R}\left(\mathrm{e}^{2 \pi t} a\right), \\
J_{R} U^{R}(a) J_{R} & =U^{R}(-a), & J_{L} U^{L}(a) J_{L}=U^{L}(-a), \\
U^{R}(a) \mathcal{B}_{A}^{R}(0, \infty) U^{R}(-a) & =\mathcal{B}_{A}^{R}(a, \infty), & & \\
U^{L}(a) \mathcal{B}_{A}^{L}(-\infty, 0) U^{L}(-a) & =\mathcal{B}_{A}^{L}(-\infty,-a) &
\end{array}
$$

where we have dropped the index $\Omega$ on the modular objects to simplify notation. Without further assumptions, $U^{R}$ and $U^{L}$ are unrelated and so are the nets $\mathcal{B}_{A}^{R}$ and $\mathcal{B}_{A}^{L}$. However, if we suppose that $(\mathrm{V})$ holds, too, then it follows from the way these unitaries are constructed (cf. [64]), that $J_{R} U^{R}(a) J_{R}=U^{L}(a), a \in \mathbb{R}$. Therefore we obtain the following:

Corollary 4.4. Under assumptions (I-IV) the nets of horizon-algebras indexed by the intervals of the half real lines,

$$
\begin{aligned}
(a, b) & \mapsto \mathcal{B}_{A}^{R}(a, b), & 0<a<b<\infty, \\
(-b,-a) & \mapsto \mathcal{B}_{A}^{L}(-b,-a), & -\infty<-b<-a<0,
\end{aligned}
$$

extend to local conformal nets $I \mapsto \mathcal{M}^{R}(I)$ and $I \mapsto \mathcal{M}^{L}(I)$ of von Neumann algebras on $S^{1}$ on the Hilbert spaces $\mathcal{H}_{0}^{R}=\overline{\mathcal{B}_{A}^{R}(a, b) \Omega}$ and $\mathcal{H}_{0}^{L}=\overline{\mathcal{B}_{0}^{L}(-b,-a) \Omega}$, respectively (where the $0<a<b<\infty$ are arbitrary).

If $(\mathrm{V})$ is assumed, too, then the net $\left(a^{\prime}, b^{\prime}\right) \mapsto \mathcal{B}_{A}\left(a^{\prime}, b^{\prime}\right)$ on the full real line extends to a local conformal net $I \mapsto \mathcal{M}(I)$ on $\mathcal{H}_{A}(\Omega)$.

Proof. The first part is a variant of Wiesbrocks's result [64, 65], cf. also [33]. We supply the relevant argument as Proposition 4A.2 in Sec. 4.3.

If assumption ( $\mathrm{V})$ is added so that $J_{R}$ intertwines $U^{R}$ and $U^{L}$, the adjoint action of $U^{R}(a)$ on the net $\mathcal{B}_{A}$ is geometrically correct, i.e. $U^{R}(a) \mathcal{B}_{A}\left(a^{\prime}, b^{\prime}\right) U^{R}(-a)=\mathcal{B}_{A}\left(a^{\prime}+\right.$ $\left.a, b^{\prime}+a\right), a \in \mathbb{R}, a^{\prime}<b^{\prime}$. Thus the net $\mathcal{B}_{A}$ together with its dilation and translation symmetries coincides with both $\mathcal{C}^{R}$ and $\mathcal{C}^{L}$ (derived from the nets $\mathcal{B}_{A}^{R}$ and $\mathcal{B}_{A}^{L}$ as in Proposition 4A.2) and their respective translation and dilation symmetries. Thus the corresponding extensions to conformally covariant theories coincide.

Condition (IV) may be viewed as a weak form of the Hawking-Unruh effect: an observer moving with the Killing flow of the bKh registers a thermal ensemble in the "vacuum" state (see [56, 62]). The term "vacuum" here means a state invariant under the space-time isometries and fulfilling additional stability conditions, in fact (IV) and (V) may be viewed as a weak form of such conditions, namely applying to the subsystem of observables concentrated on the horizon. As the group of affine translations along the geodesic generators of the horizon has positive generator derived from the modular inclusion of horizon-algebras, $\Omega$ can be justly interpreted as a vacuum vector for the horizon-algebras (cf. the principle of geometric modular action 17] or modular covariance [15]). Clearly, if $\Omega$ induces a KMS-state for the Killing flow at the Hawking temperature on $\mathcal{A}(R)$, then (IV,i) follows by Lemma 4.3. Likewise, if $\Omega$ induces a KMS-state for the Killing flow at negative Hawking temperature on $\mathcal{A}(L)$, then $(\mathrm{IV}, \mathrm{ii})$ follows by Lemma 4.3 . 
The motivation for Condition (V) is that, a horizon (or wedge) reflection symmetry should be implemented in a "vacuum" representation by the modular conjugations $J_{R}$, in analogy with the Bisognano-Wichmann result for quantum fields in Minkowski space [4, 5, 6, 56]. Our condition is actually a bit weaker in that $J_{R}$ need not implement a point-transformation of the underlying spacetime manifold. However, Condition $(\mathrm{V})$ implicitly imposes a relation between the horizon segments $\mathrm{h}_{A}(-\infty,-a)$ and $\mathrm{h}_{A}(a, \infty)$.

We finally comment on whether these assumptions are realistic. For the free scalar field, conditions (I,II) hold generally in representations induced by quasifree Hadamard states (for $\mathcal{O} \in \mathcal{K}$ based on relatively compact subsets of Cauchy surfaces, and, in more special cases, even when the base is unbounded), see [59. The HartleHawking state, i.e. the candidate for the "vacuum" state of the free scalar field on the Schwarzschild-Kruskal spacetime, should also satisfy all the assumptions [37, 40] ((III) has not been checked in the generality formulated here, but a version of (III) sufficient to imply the spin-statistics theorem in the sequel does hold). As is known from the Bisognano-Wichmann result [4], the assumptions are fulfilled for local von Neumann algebras generated by (finite-component) Wightman fields in Minkowski spacetime ((III) then holds for wedge-regions and this suffices to establish the spin-statistics relations [31, 42]). Results of Borchers [5, 6] yield (III-V) generally for algebraic quantum field theories in two spacetime dimensions. With additional conditions these generalize to higher dimensions [0, 8, [6].

Now we can formulate the conformal spin and statistics theorem. Our aim is to define the spin of a sector as the conformal spin on the horizon. To this end we need to restrict to considering sectors that are horizon localizable, namely having a representative which acts trivially on the algebras $\mathcal{B}_{A}(a, b)^{\prime}$ for some $a, b \in \mathbb{R}$ (or the same for the $B$ horizon). However this is not sufficient in general because the sector on the horizon may not be covariant. As shown in [33] covariance of localized endomorphisms with finite statistics is automatic when the net is strongly additive, which is always the case for the dual net. Unfortunately extending a sector on a conformal net to a sector on the dual net may produce soliton sectors. Therefore we shall only consider those sectors which are not only horizon localizable, but also dual localizable, namely which give rise to a localized sector on the dual net of the horizon conformal net. Clearly if we have a dual localizable sector on the net $\mathcal{O} \mapsto$ $\mathcal{A}(\mathcal{O})$ satisfying assumptions (I-V) with non-zero statistical parameter $\lambda$, we obtain a covariant sector on the dual net on the horizon with the same statistical parameter, since this is determined by the intertwiners. The following theorem is now a simple consequence of the conformal spin and statistics theorem in [32].

Theorem 4.5. Let $\mathcal{O} \mapsto \mathcal{A}(\mathcal{O})$ be a theory on a spacetime with bKh satisfying assumptions ( $\mathrm{I}-\mathrm{V})$ and $\rho$ a dual localizable sector with finite statistics. Then $\rho$ gives rise to a covariant sector on the dual net on the horizon, therefore a conformal spin $s_{\rho}$ is defined, and the conformal spin and statistics relation holds, namely $s_{\rho}=\kappa_{\rho}$. 


\section{Remarks Concluding Section 4.2}

The idea of passing from a quantum field theory initially formulated over a fourdimensional spacetime to observables concentrated on a lightlike hypersurface (i.e. pieces of a bKh) is not a new one and once was popular in quantum field theory under the keyword "infinite momentum frame". [43, 55] are just two references in this direction. This matter is studied for the first time in the operator algebraic framework in [26]. One motivation is that symmetries may be enhanced by restricting to a subtheory concentrated on a lightlike hypersurface, a particularly attractive possibility for quantum field theory in curved spacetimes where symmetries of the underlying four-dimensional spacetime are rather limited. As proved in this section, for bKh spacetimes restricting to the horizon does indeed give conformally covariant nets.

Sewell [56] was the first to observe that this allows one to formulate a BisognanoWichmann theorem relating to the Hawking effect for quantum fields on blackhole spacetimes, in the setting of a Wightman field theory (see e.g. 62 for further discussion). In this context, two papers rigorously establish related results for free field models [37, 22]. Kay and Wald [40] realized that such results may be generalized to spacetimes with a bKh and obtained strong theorems for free fields in this setting. An operator-algebraic version of aspects of Sewell's work appears in [57 where the nets $\mathcal{B}_{A}(a, b)$ are used.

We ought to mention that in general it is not very clear how "big" the algebras $\mathcal{B}_{A}(a, b)$ (or $\left.\mathcal{B}_{B}(a, b)\right)$ are in the original algebras $\mathcal{A}(\mathcal{O})$.

If $\Omega$ is cyclic for $\mathcal{B}_{A}(a, b)$ then it is resonable to expect that sectors are horizon localizable (on the $A$-horizon). Moreover in this case the conformal net on the horizon is strongly additive by definition, therefore it coincides with its dual net (cf. [33]), and then horizon-localizability and dual localizability are equivalent.

It is known that $\Omega$ is cyclic for $\mathcal{B}_{A}(a, b)$ when free fields on the $n$-dimensional Minkowski space are considered, $n \neq 2$. We give here a simple argument based on [16].

By a "free field" on Minkowski space we here mean a local net $\mathcal{A}$ of von Neumann algebras indexed by regions of Minkowski space which can be constructed by second quantization from a net $K$ of real vector spaces in a complex Hilbert space $H$, plus the usual assumptions of Poincare covariance, positive energy, and in particular the Bisognano-Wichmann property and irreducibility: $\cap_{W} \mathcal{A}(W)=\mathbb{C} I$.

Working in the first quantization space $H$ from now on, we first observe that irreducibility means $\cap_{W} K(W)=\{0\}$ and, by the Bisognano-Wichmann property, this is equivalent to there being no fixed vectors for the action of the Poincaré group on $H$.

Then, by a Theorem of Mackey (cf. e.g. [68], Proposition 2.3.5), the absence of invariant vectors for the whole Poincaré group is equivalent, when $n \neq 2$, to the absence of invariant vectors for any given translation, hence the spectrum of the generator of any light-like translation is strictly positive, i.e. zero is not an eigenvalue.

Now, given two wedges $W_{1}, W_{2}$, the cyclicity of the vacuum vector $\Omega$ for $\mathcal{A}\left(W_{1}\right) \cap$ $\mathcal{A}\left(W_{2}\right)$ is equivalent to $\left(K\left(W_{1}\right) \cap K\left(W_{2}\right)\right)+i\left(K\left(W_{1}\right) \cap K\left(W_{2}\right)\right)$ being dense in $H$, 
this being in turn equivalent to having $\left\{v \in \operatorname{dom}\left(s_{W_{1}}\right) \cap \operatorname{dom}\left(s_{W_{2}}\right): s_{W_{1}} v=s_{W_{2}} v\right\}$ dense in $H$, where $s_{W_{j}}$ denotes the "first quantized" Tomita operator defined by $s_{W_{j}}(\chi+i \phi):=\chi-i \phi, \chi, \phi \in K\left(W_{j}\right)$. When $W_{1}=\left\{(t, x): x_{1}>\left|x_{0}\right|\right\}$ and $W_{2}$ is a translation of the causal complement of $W_{1}, W_{2}=\left\{(t, x): x_{1}-c<-|t-c|\right\}, c>0$, the situation met when considering the vector space associated with the interval $(0, c)$ on the $A$-horizon, this is in turn equivalent, again by the Bisognano-Wichmann property, to the density of the space

$$
\left\{v \in \operatorname{dom}\left(\delta_{1}^{1 / 2} T(c) \delta_{1}^{1 / 2}\right): T(c) \delta_{1}^{1 / 2} T(c) \delta_{1}^{1 / 2} v=v\right\}
$$

where $a \rightarrow T(a)$ denotes the representation of the light-like translations along the $A$ horizon and $\delta_{1}$ denotes the "first quantized" modular operator for the space $K\left(W_{1}\right)$. This property clearly depends only on the restriction of the representation of the Poincaré group to the subgroup $\mathbb{P}_{1}$ generated by boosts and light-like translations with strictly positive generator (relative to the wedge $W_{1}$ ). As the logarithm of the generator of translations and the generator of the boosts give rise to (and are determined by) a representation of the CCR in one dimension, the strictly positive energy representations of $\mathbb{P}_{1}$ have a simple structure: they are always a multiple of the unique irreducible representation. Therefore the density of the space in eqn. (4.25) holds either always or never, and hence can be checked in the irreducible case. But this is the case of the current algebra on the circle, where cyclicity holds by conformal covariance.

Of course, the vector $\Omega$ is not expected to be cyclic in general for the algebra generated by the $\mathcal{B}_{A}(a, b)$, and it might even happen that $\mathcal{B}_{A}(a, b)$ contains only multiples of the identity. Field nets giving rise to non-trivial superselection sectors of the observable net localizable on the horizon can easily be constructed just by requiring the vacuum to be cyclic for the horizon field algebras. However it is not clear, in general, how strong the requirement of dual localizability is.

\subsection{Appendix to Chapter 4}

For the benefit of the non-expert reader, we present in detail in this Appendix the arguments leading from the results in [64, 65, 33] to Corollary 4.5. To begin with, we state a result about modular inclusions needed in the following.

Lemma 4A.1. Let $(\mathcal{N} \subset \mathcal{M}, \Omega)$ be a pair of von Neumann algebras with a unit vector $\Omega$ cyclic and separating for $\mathcal{M}$ and such that $\Delta^{i t} \mathcal{N} \Delta^{-i t} \subset \mathcal{N}$ for all $-t \geq 0$ (or $t \geq 0$ ), where $\Delta^{i t}, t \in \mathbb{R}$, is the modular group of $\mathcal{M}, \Omega$. Then $\mathcal{M}=\vee_{t \in \mathbb{R}} \Delta^{i t} \mathcal{N} \Delta^{-i t}$ if and only if $\Omega$ is cyclic for $\Delta^{i t} \mathcal{N} \Delta^{-i t}$ for some (hence for any) $t \in \mathbb{R}$.

Proof. If $\Omega$ is cyclic for $\Delta^{i t} \mathcal{N} \Delta^{-i t}$ for a given $t$, then it is cyclic for $\vee_{t \in \mathbb{R}} \Delta^{i t} \mathcal{N} \Delta^{-i t}$, too. However this von Neumann algebra is invariant under the modular group of $\mathcal{M}$, and hence coincides with $\mathcal{M}$ by Takesaki's theorem. Conversely, let $\xi$ be orthogonal to $\Delta^{i t} \mathcal{N} \Delta^{-i t} \Omega$. Then for any $x \in \Delta^{i t} \mathcal{N} \Delta^{-i t}$ we have $x \Omega \in \operatorname{dom}\left(\Delta^{1 / 2}\right)$, hence the function $z \mapsto\left(\Delta^{i z} x \Omega, \xi\right)$ is analytic on the strip $-i / 2<\Im z<0$ and continuous on the boundary. But as we have a + hsm inclusion, it vanishes for negative real $z$ and 
hence everywhere. Thus $\xi$ is orthogonal to $\vee_{t \in \mathbb{R}} \Delta^{i t} \mathcal{N} \Delta^{-i t} \Omega=\mathcal{M} \Omega$, completing the proof.

Proposition 4A.2. Let $(\mathcal{N} \subset \mathcal{M}, \Omega)$ be a a pair of von Neumann algebras with a unit vector $\Omega$ which is cyclic and separating for $\mathcal{M}$ and such that $\vee_{t \in \mathbb{R}} \Delta^{i t} \mathcal{N} \Delta^{-i t}=\mathcal{M}$ and $\Delta^{i t} \mathcal{N} \Delta^{-i t} \subset \mathcal{N}$ for all $-t \geq 0$, where $\Delta^{i t}, t \in \mathbb{R}$, is the modular group of $\mathcal{M}, \Omega$. Then, setting

$$
\begin{aligned}
& \mathcal{H}_{0}=\overline{\left(\mathcal { N } \cap \left(\Delta^{\left.\left.-i \mathcal{N} \Delta^{i}\right)^{\prime}\right) \Omega}\right.\right.} \\
& \mathcal{C}(a, b)=\left(\Delta^{-i \frac{\log a}{2 \pi}} \mathcal{N} \Delta^{i \frac{\log a}{2 \pi}}\right) \cap\left(\Delta^{-i \frac{\log b}{2 \pi}} \mathcal{N} \Delta^{i \frac{\log b}{2 \pi}}\right)^{\prime}\left\lceil\mathcal{H}_{0}, \quad 0<a<b,\right.
\end{aligned}
$$

the family $(a, b) \mapsto \mathrm{C}(a, b)$ extends to a local conformal net of von Neumann algebras acting on the Hilbert space $\mathcal{H}_{0}$.

Proof. Set

$$
\mathcal{N}_{a}=\Delta^{-i \frac{\log a}{2 \pi}} \mathcal{N} \Delta^{i \frac{\log a}{2 \pi}}, \quad a>0
$$

By the previous lemma $\Omega$ is cyclic for $N_{a}, a>0$, therefore we may apply a result of Wiesbrock and Araki-Zsido ([64, 1]) to the + hsm inclusion $(\mathcal{N} \subset \mathcal{M}, \Omega)$ and get a one parameter group of unitaries $U(a)$ on $\mathcal{H}$ with positive generator satisfying

$$
\begin{aligned}
\Delta^{-i t} U(a) \Delta^{i t} & =U\left(e^{2 \pi t} a\right) \\
J U(a) J & =U(-a)
\end{aligned}
$$

Hence we have

$$
\mathcal{N}_{a}=U(a) \mathcal{M} U(a)^{*}, \quad a \geq 0,
$$

and this equation is used to define $\mathcal{N}_{a}$ for negative $a$.

We now set

$$
\begin{aligned}
\mathcal{C}(a, b) & =\mathcal{N}_{a} \cap \mathcal{N}_{b}^{\prime} \uparrow \mathcal{H}_{0}, & -\infty<a<b<+\infty \\
\mathcal{C}(-\infty, b) & =\vee_{a<b} \mathcal{C}(a, b), & -\infty<b<+\infty \\
\mathcal{C}(a,+\infty) & =\vee_{b>a} \mathcal{C}(a, b), & -\infty<a<+\infty
\end{aligned}
$$

and the definition of $\mathcal{C}(a, b)$ clearly agrees with (4.26) when $0<a<b<\infty$. Furthermore,

$$
\mathcal{H}_{0}=\overline{\mathcal{N} \cap \mathcal{N}_{e^{2 \pi}}^{\prime} \Omega}=\overline{\mathrm{C}\left(1, e^{2 \pi}\right) \Omega} .
$$

Moreover, the operators $J, \Delta$ restricted to $\mathcal{H}_{0}$ give the modular conjugation and operator of $(\mathcal{C}(0, \infty), \Omega)$. Similarly, using the results of [64, 1] anew, the restriction of $U(a)$ to $\mathcal{H}_{0}$ (again denoted by $U(a)$ ) coincides with the unitary group derived from the + hsm inclusion $(\mathfrak{C}(1, \infty) \subset \mathcal{C}(0, \infty), \Omega)$. Now a standard Reeh-Schlieder argument, based on the positivity of the generator of $U(a)$, shows that $\overline{\mathfrak{C}(-\infty, b) \Omega}$ is independent of $b$, while the "modular" Reeh-Schlieder argument in Lemma 4A.1 shows that $\overline{\mathrm{C}(a, b) \Omega}$ is independent of $a \in(-\infty, b)$. Thus the inclusion $(\mathrm{C}(1, \infty) \subset$ $\mathcal{C}(0, \infty), \Omega)$ is standard. We have proved that $\mathcal{H}_{0}=\overline{\mathfrak{C}(a, b) \Omega}$ for any $-\infty \leq a<b \leq$ $+\infty$, and that $\mathcal{C}$ gives a translation-dilation covariant net of von Neumann algebras on $\mathcal{H}_{0}$. Then we get a conformally covariant net by a result of Wiesbrock ([65], see also [33]). 


\section{$5 \quad$ The Spin and Statistics Relation for Spacetimes with Rotation Symmetry}

In this section, we present a proof of the spin and statistics relation for superselection sectors on a globally hyperbolic spacetime with some rotational symmetry.

The main assumption here is the existence of a suitable family of regions, called wedges, each being equipped with a reflection mapping it to its causal complement and of a net of von Neumann algebras with a common cyclic vector whose modular conjugations implement the said reflections, in the spirit of [18] and [45].

Moreover we assume the existence of rotational spacetime symmetries, rotating a wedge to its causal complement and belonging to the commutator of the spacetime symmetry group. As we shall see, our geometric assumptions are satisfied in many interesting spacetimes and form the geometric basis for the rotational spin and statistics theorem, explained in more detail below.

\subsection{Geometric Assumptions}

A spacetime with rotation and reflection symmetry is a quadruple $\left(M, \mathcal{W}, G_{+}, \mathbf{j}\right)$, where $M$ is a globally hyperbolic spacetime, $\mathcal{W}$ is a family of open subregions called wedges, $G_{+}$is a Lie group of proper (i.e. orientation preserving) transformations of $M$ and $\mathbf{j}$ is a map from $\mathcal{W}$ to the antichronous (i.e. time reversing) reflections in $G_{+}$; we write it as $W \mapsto \mathbf{j}_{W}$. We denote the orthochronous subgroup of $G_{+}$by $G_{+}^{\uparrow}$ and the identity component of $G_{+}$by $G_{0}$. The universal covering of $G_{0}$ is denoted by $\widetilde{G}$. The $\mathbb{Z}_{2}$ action implemented by any $\mathbf{j}_{W}$ on $G_{0}$ lifts to an action on $\widetilde{G}$. The quadruple has to satisfy the following properties:

(a) $\mathbf{j}$ leaves $\mathcal{W}$ globally invariant and verifies $\mathbf{j}_{W}(W)=W^{\perp}$ and $\mathbf{j}_{g W}=g \mathbf{j}_{W} g^{-1}$, $W \in \mathcal{W}, g \in G_{+}$.

(b) There is $W \in \mathcal{W}$ and an element $\mathfrak{h}$ in the Lie algebra of $G_{0}$ such that

(1) $\exp (2 \pi \mathfrak{h})$ is the identity in $G_{0}$,

(2) $\mathbf{j}_{W} \exp (t \mathfrak{h}) \mathbf{j}_{W}=\exp (-t \mathfrak{h})$,

(3) $\exp (\pi \mathfrak{h}) W=W^{\perp}$,

(4) $\cap_{0 \leq t \leq \pi / 2} \exp (t \mathfrak{h}) W$ is non-empty.

(c) $\mathfrak{h}$ belongs to the commutator of the Lie algebra of $G_{0}$.

Remark 5.1. Two wedges $W, \widetilde{W}$ are called orthogonal if $\mathbf{j}_{W} \widetilde{W}=\widetilde{W}$ and $\mathbf{j}_{\widetilde{W}} W=W$. It is easy to see that $W$ and $\exp \left(\frac{\pi}{2} \mathfrak{h}\right) W$ are orthogonal. Indeed, making use of assumptions (b 2) and (b 3), we get

$$
\begin{aligned}
\mathbf{j}_{W} \exp \left(\frac{\pi}{2} \mathfrak{h}\right) W & =\exp \left(-\frac{\pi}{2} \mathfrak{h}\right) \mathbf{j}_{W} W \\
& =\exp \left(-\frac{\pi}{2} \mathfrak{h}\right) W^{\perp}=\exp \left(-\frac{\pi}{2} \mathfrak{h}\right) \exp (\pi \mathfrak{h}) W \\
& =\exp \left(\frac{\pi}{2} \mathfrak{h}\right) W .
\end{aligned}
$$


The second equation is proved analogously.

We shall also consider spaces where property (c) is replaced by the following property:

$\left(c^{\prime}\right)$ There exists a wedge $\widetilde{W}$, orthogonal to $W$, such that $\mathbf{j}_{\widetilde{W}}$ commutes with $\exp (t \mathfrak{h})$.

Remark 5.2. (i) Assumption (a) has to be seen as a part of the definition of a wedge. The first part of property (a) says that any wedge is $G_{+}$-equivalent to its causal complement, hence a wedge is in some sense "a half" of $M$ or, more precisely, is the causal completion of "a half" of a Cauchy surface. The second part means that $\mathbf{j}_{W}$ commutes with the stabilizer of $W$ and, when $G_{+}$acts transitively on $\mathcal{W}$, says that $\mathbf{j}$ is determined by its value on one wedge.

(ii) Properties (b) describe the rotation symmetry. In view of property (b 1) we call the group elements $\exp (t \mathfrak{h})$ rotations. Property (c) ensures that all characters of $\widetilde{G}$ are trivial on the cycle $\{\exp (t \mathfrak{h}), t \in[0,2 \pi]\}$, since the latter belongs to the commutator subgroup of $\widetilde{G}$ where all characters are trivial. As we shall see, this makes the spin well defined.

(iii) The element $\mathbf{j}_{W}$, seen as an automorphism of the Lie algebra of $G_{0}$, has eigenvalues 1 and -1 and by (a) the eigenspace corresponding to 1 consists of generators of transformations preserving $W$. Therefore (b 2) essentially says that not all rotations preserve $W$. More precisely, $W$ may be rotated to its spacelike complement by (b 3). (iv) Property (b 3) mainly expresses the fact that $2 \pi$ is the minimal period of the one-parameter group $\exp (t \mathfrak{h})$.

(v) Property (b) is stated for one wedge $W$, but then holds for any wedge in the family $\mathcal{W}_{0}:=\left\{g W: g \in G_{+}\right\}$. We are of course interested in the case where the cycle $\{\exp (t \mathfrak{h}), t \in[0,2 \pi]\}$ is not homotopy trivial and hence gives rise to a non-trivial notion of spin. However this is not needed for the proof of the spin and statistics theorem nor do we require that the $\exp (2 \pi \mathfrak{h})$ generate the homotopy group of $G_{0}$.

(vi) Property $\left(\mathrm{c}^{\prime}\right)$ implies that $W, e^{\pi / 2 \mathfrak{h}} W$ and $\widetilde{W}$ are mutually orthogonal. It also implies that $r_{W}:=\mathbf{j}_{W} \mathbf{j}_{\widetilde{W}}$ is an involution in $G_{+}^{\uparrow}$ and that $\exp (2 t \mathfrak{h})=\left[\exp (t \mathfrak{h}), r_{W}\right]$, where the square brackets here denote the multiplicative commutator. As a consequence, the rotations $\exp (t \mathfrak{h})$ belong to the commutator subgroup of $G_{+}^{\uparrow}$. In this sense $\left(\mathrm{c}^{\prime}\right)$ is a weak form of $(\mathrm{c})$. $G_{+}^{\uparrow}$ and $G_{0}$ do not always coincide. Of course $r_{W} \in G_{+}^{\uparrow}$, but we do not require that $r_{W}$ belongs to $G_{0}$.

(vii) Property (b) fixes the the generator $\mathfrak{h}$ up to a sign, indeed (b 1) fixes the generator up to an integer, (b 3) implies this integer to be odd, and (b 5) requires this integer to be 1 or -1 . When the spacetime is two-dimensional, i.e. when the Cauchy surface is 1-dimensional, the orientation fixes a direction on any spacelike curve (from left to right). In this case we choose the sign in such a way that the element $\mathfrak{h}$ generates a rotation in the prescribed direction.

\section{Assumptions (a), (b), (c) and ( $\left.\mathrm{c}^{\prime}\right)$ in some spacetimes}

In the case of the $n$-dimensional Minkowski spacetime $M^{n}$, a wedge is any $G_{+}-$ transform of the region $W=\left\{\left|x_{0}\right|<x_{1}\right\}$ if $n>2$, and of the region $\{x>0\}$ if $n=1$. 
Taking $\mathbf{j}_{W}$ to be the reflection w.r.t. the edge of the wedge, the map $\mathbf{j}$ turns out to be uniquely defined by property (a).

When $G_{+}$is the proper Poincaré group and $n \geq 3$, property (b) holds with $W$ as above and $\mathfrak{h}$ as the generator of rotations in the $\left(x_{0}, x_{1}\right)$-plane. Indeed the proper orthochronous Poincaré group is perfect, hence property (c) is obviously satisfied. If $n \geq 4$ then $\left(\mathrm{c}^{\prime}\right)$ is satisfied too, with $\widetilde{W}=\left\{\left|x_{0}\right|<x_{2}\right\}$.

When $G_{+}$is the proper conformal group, properties (b) and (c) are satisfied for any $n \geq 1, \mathfrak{h}$ being the generator of a suitable group of (conformal) rotations. Property $\left(\mathrm{c}^{\prime}\right)$ is satisfied when $n \geq 3, W$ being as before, $\mathfrak{h}$ being the generator of rotations in the $\left(x_{0}, x_{1}\right)$-plane and $\widetilde{W}$ a double cone with spherical basis centred on the origin.

Since the $n$-dimensional de Sitter spacetime $D^{n}$ may be defined as the hyperboloid $x_{0}^{2}+1=|\mathbf{x}|^{2}$ in $M^{n+1}$, the wedges can be defined as the intersection of this hyperboloid with the wedges in $M^{n+1}$ whose edge contains the origin. Then properties (b), (c) or $\left(\mathrm{c}^{\prime}\right)$ hold for $D^{n}$ if and only if properties (b), (c) or $\left(\mathrm{c}^{\prime}\right)$ hold for $M^{n+1}$ (with Poincaré symmetry), respectively.

Note that the Cauchy surface of $D^{n}$ is compact and the same is true for $M^{n}$ with conformal symmetry, since in this case the quantum field theories actually live on (a covering of) the Dirac-Weyl compactification of $M^{n}$ (cf. [14).

Whenever the spin makes sense in the above examples, i.e. whenever (c) or $\left(c^{\prime}\right)$ holds, the group $G_{+}^{\uparrow}$ has no non-trivial finite dimensional representations, a much stronger requirement than $(c)$ or $\left(c^{\prime}\right)$. In this case the spin and statisitcs relation may be proved as in 45].

Moreover, in these examples, modular covariance makes sense, i.e. there is a natural definition of the geometric action of $\Delta^{i t}$, furthermore, the Bisognano-Wichmann property has been proved for Wightman fields ([4, 10]), wedges separate spacelike points and every double cone is an intersection of wedges. Therefore geometric modular conjugation follows from modular covariance (as in [31], cf. [18]) and modular covariant free fields may be constructed canonically as in [16] by second quantizing (anti-)unitary representations of $G_{+}$.

We now describe a class of spacetimes where these additional features do not hold, namely where the group admits one-dimensional representations and the wedges do not separate points. Nevertheless, these cases are still covered by the spin and statistics theorem we are going to present below. 


\section{Spherically symmetric black holes}

We call spherically symmetric black holes those spacetimes $\left(K, g_{K}\right)$ whose structure is very similar to the Schwarzschild-Kruskal spacetime, i.e. they are isometric to $X \times S^{n}$, $X$ being the set of points $\left(x_{0}, x_{1}\right) \in \mathbb{R}^{2}$ with $x_{0}^{2}-x_{1}^{2}<\mu^{2}, \mu \in \mathbb{R} \cup\{\infty\}$, with the metriç

$$
d s_{K}^{2}=a\left(x_{0}^{2}-x_{1}^{2}\right)\left(d x_{0}^{2}-d x_{1}^{2}\right)-b\left(x_{0}^{2}-x_{1}^{2}\right) d^{2} \sigma,
$$

where $d^{2} \sigma$ is the usual Riemannian metric on the sphere $S^{n}$ and $a$ and $b$ are smooth, strictly positive functions. Then the hypersurface $x_{0}=0$ is a Cauchy surface and $\left(K, g_{K}\right)$ is globally hyperbolic. The structure of such spacetimes is in some respects similar to that of Minkowski spacetime. For instance, if points in $X \times S^{n}$ are represented as $\left(x_{0}, x_{1}, \sigma\right)$, then one may define a one-parametric group of isometries $\Lambda_{t}$, $t \in \mathbb{R}$, by replacing the pair $\left(x_{2}, x_{3}\right) \in \mathbb{R}^{2}$ by $\sigma \in S^{n}$ in definition (4.2) and then define $\Sigma$ and $\mathrm{h}_{A}$ and $\mathrm{h}_{B}$, correspondingly. Hence $\left(K, g_{K}\right)$ has the structure of a spacetime with $\mathrm{bKh}$, where the Killing flow is $\tau_{t}=\Lambda_{t}, t \in \mathbb{R}$. Moreover, there is an horizon reflection $\mathbf{j}\left(x_{0}, x_{1}, \sigma\right)=\left(-x_{0},-x_{1}, \sigma\right)$ which is a PT symmetry, i.e. an orientation and chronology-reversing isometry.

Let us investigate further the isometries of such spacetimes. To simplify the matter a bit, we assume that $\left(K, g_{K}\right)$ does not admit translations in the $X$-part of $K=X \times S^{n}$ as symmetries. (This is not really a restriction; our findings can be modified by taking the semidirect product of the translational symmetry group $T_{X}$ with the non-translational symmetry group $G$ in the presence of such symmetries. For our treatment of the connection between rotational spin and statistics, translational symmetries are irrelevant.) Since $\left(K, g_{K}\right)$ is orientable and time-orientable, we consider the groups $G_{+}$and $G_{+}^{\uparrow}$ of proper (i.e. orientation preserving) and proper orthochronous (i.e. time-orientation preserving) isometries, respectively. In the following, we describe the proper orthochronous subgroup $G_{+}^{\uparrow}$.

The form of the metric tensor $g_{K}$ and the assumed triviality of $T_{K}$ imply that all isometries leave $\Sigma$ globally fixed and that an element of $G_{+}^{\uparrow}$ acting trivially on $\Sigma$ has to be an element of the Killing flow. Conversely, orientation preserving isometries of $\Sigma$, i.e. elements of $S O(n+1)$, naturally give rise to symmetries in $G_{+}^{\uparrow}$. Indeed, $\hat{R}\left(x_{0}, x_{1}, \sigma\right)=\left(x_{0}, x_{1}, R \sigma\right), R \in S O(n+1)$, gives an isometry of $\left(K, g_{K}\right)$. To extend orientation reversing isometries of $\Sigma$ to orientation preserving isometries of $K$, we obviously need a different procedure. To this end we note that each orientation reversing isometry of $\Sigma \equiv S^{n}$ can be written as a product of a rotation in $S O(n+1)$ and an equatorial reflection $r_{Q}$, where $Q$ denotes the $S^{n-1}$ equator of fixed points of such a reflection. More precisely, $r_{Q}$ reflects points on $S^{n}$ about $Q$ along the great circles orthogonal to the equator $Q$. In other words, $r_{Q}$ acts as a reflection of the normal geodesic spray of $Q$ in $S^{n}$. Note that such equatorial reflections generate the action of $O(n+1)$ on $S^{n}$. In fact, if an equator $Q_{1}$ is inclined at angle $\phi$ to an equator $Q_{2}$, then $r_{Q_{1}} r_{Q_{2}}$ is a rotation by $2 \phi$ about the axis defined by the intersection of $Q_{1}$ and $Q_{2}$.

\footnotetext{
${ }^{8}$ It is customary to write coordinate indices as upper indices, but our deviating from this convention is unlikely to cause confusion.
} 
Now choosing a normalized, timelike, future-oriented, rotation-invariant normal vector field $\xi_{0}$ along $\Sigma$ there is a unique normalized, spacelike, outward-oriented, rotation invariant normal vector field $\xi_{1}$ along $\Sigma$ such that $\xi_{0}+\xi_{1}$ is parallel to the vector field $\chi_{A}$. It is therefore equivalent to choosing an orthonormal frame on the $X$-component of $K=X \times S^{n}$. Moreover the Killing flow acts transitively on the set of possible such choices.

An equatorial reflection $r_{Q}$ extends to an orientation and chronology-preserving isometry $\hat{r}_{Q, 0} \in G_{+}^{\uparrow}$ by setting $\hat{r}_{Q, 0}:=\left(x_{0},-x_{1}, r_{Q} \sigma\right)$ and we define $\hat{r}_{Q, t}:=\Lambda_{t} \hat{r}_{Q, 0} \Lambda_{t}^{-1}$, where $\Lambda_{t}, t \in \mathbb{R}$, is the Killing flow. Each $\hat{r}_{Q, t} \in G_{+}^{\uparrow}$ is an involution. On the other hand, by the above observation, each involution in $G_{+}^{\uparrow}$ restricting to some $r_{Q}$ on $\Sigma$ must be of the form $\hat{r}_{Q, t}$ for some $t \in \mathbb{R}$. Clearly, $\hat{r}_{Q, t}$ determines a unique normalized, spacelike, outward-oriented, rotation invariant normal vector field $\xi_{1}$ along $\Sigma$ which is anti-invariant under $\hat{r}_{Q, t}$.

Thus $G_{+}^{\uparrow}$ is generated by the Killing flow, the (extensions of the) orientation preserving isometries of $\Sigma$ and the reflections $\hat{r}_{Q, t}$ so that $G_{+}^{\uparrow} \equiv(\mathbb{R} \times S O(n+1)) \times_{\sigma} \mathbb{Z}_{2}$, where $\sigma$ denotes the conjugation by $\hat{r}_{Q, 0}$, for some given equator $Q$. Consequently $G_{0} \equiv(\mathbb{R} \times S O(n+1))$ and $G_{+}$, being generated by $G_{+}^{\uparrow}$ and the horizon reflection $\mathbf{j}$, is isomorphic to $(\mathbb{R} \times S O(n+1)) \times_{\sigma} \mathbb{Z}_{2} \times \mathbb{Z}_{2}$. The following lemma obviously holds.

Lemma 5.3. On a spherically symmetric black hole, the commutator subalgebra of the Lie algebra of the identity component $G_{0}$ of the group of proper isometries is isomorphic to so $(n+1)$. The commutator subgroup of $G_{0}$ is isomorphic to $S O(n+1)$.

We now show that the reflection symmetries $\hat{r}_{Q, t}$ are naturally associated with wedge-like subregions of $K$. Indeed, given a normalized, spacelike, outward-oriented, rotation-invariant normal vector field $\xi_{1}$ along $\Sigma$, its (two-sided, maximally extended) geodesic spray gives a geodesic-foliated Cauchy surface containing $\Sigma$, and it is easy to see that all such Cauchy surfaces arise in this way. Therefore, given a reflection $\hat{r}_{Q, t}$ and an open hemisphere $E$ in $\Sigma \equiv S^{n}$ with $\partial E=Q$, we may consider the open causal completion $W(E, t)$ of the part of the Cauchy surface generated by the spacelike vectors determined by $\hat{r}_{Q, t}$ and based on $E$. Put differently, defining $\hat{E}_{0}:=$ $\left\{\left(0, x_{1}, \sigma\right): x_{1} \in \mathbb{R}, \sigma \in E\right\}$ and $\hat{E}_{t}:=\Lambda_{t} \hat{E}_{0} \Lambda_{t}^{-1}, t \in \mathbb{R}$, then $W(E, t)=\operatorname{int} D\left(\hat{E}_{t}\right)$ where $D\left(\hat{E}_{t}\right)$ is the domain of dependence of $\hat{E}_{t}$. We also mention that the edge of the wedge $W(E, t)$ is the spacelike cylinder generated by the geodesic spray of the vectors of $\xi_{1}$ based on $\partial E$, i.e. the set $\Lambda_{t}\left\{\left(0, x_{1}, \sigma\right): x_{1} \in \mathbb{R}, \sigma \in \partial E\right\}$. Hence each $W(E, t)$ is a diamond. The set of such wedge-regions will be denoted by $\mathcal{W}_{0}$. The following proposition immediately follows.

Proposition 5.4. (i) $W(E, t)^{\perp}=\hat{r}_{\partial E, t} W(E, t)=W\left(E^{\prime}, t\right)$, where $E^{\prime}$ denotes the interior of the complement of $E$.

(ii) $\hat{R} W(E, t)=W(R E, t)$, for any $R \in S O(n+1)$.

(iii) $\Lambda_{s} W(E, t)=W(E, s+t)$.

(iv) The group $G_{+}^{\uparrow}$ acts transitively on the family $\mathcal{W}$ of wedges $W(E, s)$.

(v) The group $G_{+}^{\uparrow}$ is generated by the reflections $\hat{r}_{Q, t}$. 
Now we show that these spacetimes fit in the scheme proposed at the beginning of this section. Let us define $\mathcal{W}$ as $\mathcal{W}_{0} \cup\{R\} \cup\{L\}, \mathbf{j}_{R}=\mathbf{j}_{L}$ as the horizon reflection $\mathbf{j}$ and $\mathbf{j}_{W(E, t)}=\mathbf{j}_{R} \hat{r}_{\partial E, t}$.

Proposition 5.5. If $n \geq 2$ then properties (a), (b), (c) and ( $\left.c^{\prime}\right)$ hold. If $n=1$ then properties (a), (b) and ( $\left.c^{\prime}\right)$ hold.

Proof. Proposition 5.4 immediately gives (a). Then let $W=W(E, t)$ and choose $\mathfrak{h} \in \operatorname{so}(n+1)$ as an eigenvector with eigenvalue -1 of $\mathbf{j}_{W(E, t)}$, normalized in such a way that $\exp (\vartheta \mathfrak{h})$ is a rotation through an angle $\vartheta$. Then property (b) is obviously satisfied and choosing $\widetilde{W}=R$ we get property $\left(\mathrm{c}^{\prime}\right)$. When $n \geq 2$, (c) follows by Lemma 5.3.

\subsection{Quantum Field Theories on Spacetimes with Rotation Symmetry}

Now we consider a net $\mathcal{O} \mapsto \mathcal{A}(\mathcal{O})$ of von Neumann algebras indexed by elements $\mathcal{O} \in \mathcal{K} \cup \mathcal{W}$ where $\mathcal{K}$ is the set of regular diamonds and $\mathcal{W}$ is a set of wedges with the properties discussed in the previous section; this net describes the observables of a local quantum theory on $M$. We require irreducibility, additivity and Haag duality as in assumptions (I-III) of Sect. 4.2 and, moreover,

(VI) Reeh-Schlieder property: There exists a unit vector $\Omega$ (vacuum) cyclic for the von Neumann algebras associated with all wedge regions.

(VII) Geometric modular conjugation:

$$
J_{W} \mathcal{A}(\mathcal{O}) J_{W}=\mathcal{A}\left(\mathbf{j}_{W} \mathcal{O}\right)
$$

where $\mathcal{O}$ is any regular diamond and $J_{W}$ denotes the modular conjugation associated with the algebra $\mathcal{A}(W)$ and the vector $\Omega$, cf. Sect. 4.2 .

(VIII) Covariance: There exists a unitary representation $U$ of the group $G_{+}^{\uparrow}$ such that $U(g) \Omega=\Omega$ for any $g \in G_{+}^{\uparrow}, U(g) \mathcal{A}(\mathcal{O}) U(g)^{*}=\mathcal{A}(g \mathcal{O})$ for any $g \in G_{+}^{\uparrow}$ and any regular diamond $\mathcal{O}$ and $J_{W} U(g) J_{W}=U\left(\mathbf{j}_{W} g \mathbf{j}_{W}\right)$ for any wedge $W$.

Let us note that, under the previous hypotheses, the representation $U$ extends to an (anti)-unitary representation of $G_{+}$with a geometric action on the net verifying $U\left(\mathbf{j}_{W}\right)=J_{W}$.

Proposition 5.6. Under the above assumptions, the net satisfies duality for the relation $\hat{\perp}$, namely

$$
\mathcal{A}(\mathcal{O})=\bigcap_{\mathcal{O}_{1} \hat{\perp} \mathcal{O}} \mathcal{A}\left(\mathcal{O}_{1}\right)^{\prime}
$$

where (cf. Appendix to Section 3) $\mathcal{O}_{1} \hat{\perp} \mathcal{O}$ if $\mathcal{O}_{1} \perp \mathcal{O}$ and $\exists \mathcal{O}_{2} \in \mathcal{K}: \mathcal{O}_{1}, \mathcal{O} \subset \mathcal{O}_{2}$. 
Proof. Let $\mathcal{O}_{1} \perp \mathcal{O}$. By Lemma 2.1, for any $x \in \mathcal{O}_{1}$ there exist $\mathcal{O}_{x}, \tilde{\mathcal{O}}_{x} \in \mathcal{K}$ such that $\mathcal{O} \perp \mathcal{O}_{x}$ and $\mathcal{O}, \mathcal{O}_{x} \subset \tilde{\mathcal{O}}_{x}$, in particular $\mathcal{O} \hat{\perp} \mathcal{O}_{x}$. Then

$$
\mathcal{A}(\mathcal{O}) \subseteq \cap_{\mathcal{O}_{1} \perp \mathcal{O}} \cap_{x \in \mathcal{O}_{1}} \mathcal{A}\left(\mathcal{O}_{x}\right)^{\prime}=\cap_{\mathcal{O}_{1} \perp \mathcal{O}} \mathcal{A}\left(\mathcal{O}_{1}\right)^{\prime}=\mathcal{A}(\mathcal{O})
$$

where the first equality follows by additivity and the second by duality.

(IX) equivalence of local and global intertwiners: Given a representation $\pi$ satisfying the selection criterion and localized in a wedge $W$, let $\rho_{W}$ denote the associated endomorphism of $\mathcal{A}(W)$, then

$$
(\pi, \pi)=\left(\rho_{W}, \rho_{W}\right)
$$

Remark 5.7. ( $i$ ) This assumption implies factoriality for the algebras associated with wedge regions, that irreducibility of representations coincides with irreducibility on a wedge and that the equality $\left(\pi, \pi^{\prime}\right)=\left(\rho_{W}, \rho_{W}^{\prime}\right)$ holds for pair of representations (see [32]).

(ii) Assumption (IX) has been shown to follow from dilation invariance [54, and it is conjectured that it already follows from the existence of a non-trivial scaling limit. We give an explicit proof of its validity for Minkowski space of any dimension in the Appendix to this section.

(iii) If we assume $G_{+}^{\uparrow}$ to be continuously represented by automorphisms $\alpha_{g}, G_{+}$to be generated by $\left\{\mathbf{j}_{W}, W \in \mathcal{W}\right\}$ and $\mathrm{Ad} J_{W_{1}} J_{W_{2}}=\alpha_{g}$, with $g=\mathbf{j}_{W_{1}} \mathbf{j}_{W_{2}}$, we get covariance (VIII). Moreover we obtain algebraic covariance for any sector with finite statistics, namely $\rho \simeq \alpha_{g} \rho \alpha_{g}^{-1}, g \in G_{+}^{\uparrow}$. By an argument of Müger [18, this implies that any sector is covariant w.r.t. a continuous representation of a central extension of $G_{+}^{\uparrow}$. When the wedges separate spacelike points, i.e. regular diamonds are intersections of wedges, geometric modular conjugation (VII) also follows (cf. [18]).

\section{Spin and Statistics under property (c)}

Theorem 5.8. Let $\pi$ be a representation satisfying the selection criterion and localized in $\mathcal{O} \subset W$. Suppose the associated endomorphism $\rho_{W}$ of the von Neumann algebra of the wedge $W$ has finite index. Let $j$ be the antilinear morphism implemented by the modular conjugation of $(\mathcal{A}(W), \Omega)$. Then $j \cdot \pi \cdot j$ is a conjugate of $\pi$ and $\pi$ has finite statistics.

Remark 5.9. To inclusions of von Neumann algebras one can assign an invariant, a positive number called the index (cf. 44] and refs. cited there). The index of the endomorphism $\rho_{W}$ is that assigned to the inclusion $\rho_{W}(\mathcal{A}(W)) \subset \mathcal{A}(W)$. For discussion of the relation between the statistical dimension of a superselection sector in quantum field theory in Minkowski spacetime and the index of its associated localized endomorphisms, the reader is again referred to [44]. 
Proof. Pick a representation $\pi^{\prime}$ equivalent to $\pi$ and localized in $W^{\perp}$. Then arguing as in [30], we see that $j \pi j$ and $\pi^{\prime}$ yield conjugate endomorphisms of the von Neumann algebra of the wedge $W^{\perp}$. The next step is to deduce from Assumption IX that $j \pi j$ and $\pi$ are conjugate representations. This circumstance is obscured by the fact that the product even of localized representations is defined only up to equivalence. For this reason, we use cocycles from $Z_{t}^{1}(\mathcal{A})$ instead of representations, recalling Theorem 3A.5. We have a faithful tensor ${ }^{*}$-functor $Z_{t}^{1}(\mathcal{A}) \rightarrow \mathcal{T}(a)$ taking a cocycle $z$ into the associated endomorphism $y(a)$ in $a$ and an arrow $t$ into $t_{a}$. If $a \subset W$, then there is a tensor ${ }^{*}$-functor from $\mathcal{T}(a)$ into the category of endomorphisms of the von Neumann algebra of the wedge $W$, mapping an object $\rho$ onto its restriction to the algebra of the wedge $\rho_{W}$ and acting as the identity on arrows. Assumption IX means that the composition of these functors is even full. Thus if $y(a)_{W}$ and $\bar{y}(a)_{W}$ are the images of $z$ and $\bar{z}$ and are conjugates, $z$ and $\bar{z}$ are conjugates. If $z$ is a cocycle associated with $\pi^{\prime}$ and $\bar{z}$ is a cocycle associated with $j \pi j$, then the endomorphisms of $\mathcal{A}\left(W^{\perp}\right)$ obtained by restriction are conjugates and so are the equivalent endomorphisms $y(a)_{W}$ and $\bar{y}(a)_{W}$. Hence $z$ has a left inverse and finite statistics.

By assumption, $J_{W}$ implements a spacetime reflection consisting of a time reversing (since $J_{W}$ is anti-unitary) and a space reversing transformation since, preserving the overall orientation, it has to reverse the orientation of any globally invariant Cauchy surface. Therefore the previous theorem is indeed a PCT theorem.

In the following we choose a rotationally symmetric spacetime $\left(M, \mathcal{W}, G_{+}, \mathbf{j}\right)$ satisfying properties (a), (b) and (c), a local net $\mathcal{O} \mapsto \mathcal{A}(\mathcal{O})$ verifying the above assumptions and an irreducible, $\widetilde{G}$-covariant, superselection sector with finite statistics.

If $\pi$ is a representation obeying the selection criterion with finite statistics, as above, let $\rho$ be a localized endomorphism defined using an associated cocycle. The standard left inverse for the cocycle gives us a left inverse $\phi$ for $\rho$, cf. Lemma 3A.10. When the statistics operator $\varepsilon(\rho, \rho)$ is uniquely defined, namely when the space-time dimension is greater than or equal to $3, \phi_{\rho, \rho}(\varepsilon(\rho, \rho))$ is an intertwiner between $\pi$ and itself. Therefore, when $\pi$ is irreducible, it is a complex number, cf. Sec. 3.4.

When the dimension of a Cauchy surface is one, there are two choices for the statistics and correspondingly two choices for the statistics parameter. In this case, we choose the statistics operator $\varepsilon$ associated with the connected component of $\mathcal{G}^{\perp}$ where the 1-simplices have the chosen orientation (cf. Remark 5.2 (vii)).

Let us note that, by Assumption IX, a left inverse exists even when a Cauchy surface is compact.

The preceding theorem shows that the statistics phase is well defined. In fact, the same is true for the spin, as the following proposition shows.

Proposition 5.10. Let $\pi$ be a representative of the given sector and $\left(\pi, U_{\pi}\right)$ a covariant representation. Then:

(i) The quantity $s:=U_{\pi}(\exp (2 \pi \mathfrak{h}))$ is a complex number of modulus one depending only on the equivalence class of $\pi$ and not on the representation $U_{\pi}$. It is called the spin of the sector. 
(ii) Given $U_{\pi}$, let $\nu:=\operatorname{Ad} V \cdot \pi$ be an equivalent representation, then $\left(\nu, U_{\nu}\right)$ is a covariant representation, where

$$
U_{\nu}:=V U_{\pi} V^{*}
$$

does not depend on the intertwiner $V$.

Proof. (i) Since $\pi$ is irreducible, $U_{\pi}$ is fixed up to a one-dimensional representation. By Assumption (c), one-dimensional representations are trivial on $\exp (t \mathfrak{h})$, hence $U_{\pi}(\exp (2 \pi \mathfrak{h}))$ does not depend on the chosen representation. Since $\exp (2 \pi \mathfrak{h})$ is the identity element in $G_{0}$, the corresponding element in $\tilde{G}$ is a central element, so $U_{\pi}(\exp (2 \pi \mathfrak{h})$ is a scalar by irreducibility. Equation 5.2 shows that $s$ does not depend on the representative $\rho$. (ii) is obvious.

A priori $s$ depends on the Lie algebra element $\mathfrak{h}$. However, this possibility is ruled out a posteriori by the spin and statistics relation. In the following, we fix the assignment $\pi \mapsto U_{\pi}$ for any representative $\pi$, as described in the above proposition.

Now we may state the main theorem of this section. The proof will require some lemmas.

Theorem 5.11. Let us consider a local net $\mathcal{O} \mapsto \mathcal{A}(\mathcal{O})$ on a rotationally symmetric spacetime $\left(M, \mathcal{W}, G_{+}, \mathbf{j}\right)$, satisfying the above assumptions (I-III), (VI-IX), and an irreducible $\tilde{G}$-covariant superselection sector with finite statistics on such a net. Then the spin of the sector agrees with its statistics phase.

Let $\pi$ be a representative of a sector with finite statistics, let $\mathcal{O}$ be contained in a wedge $W$ and let $\rho$ be an object of $\operatorname{End} \mathcal{A}(\mathcal{O})$ associated with $\pi$ and set

$$
\bar{\rho}:=j \cdot \rho \cdot j
$$

where $j$ is the modular antilinear morphism associated with $\mathcal{A}(W)$ and $\Omega . \bar{\rho}$ is an object of End $\mathcal{A}\left(\mathbf{j}_{W} \mathcal{O}\right)$. Let $V$ denote the Araki-Connes-Haagerup standard implementation (cf. e.g. 32]) of the restriction of $\rho$ to $\mathcal{A}(W)$.

Lemma 5.12. (cf. Lemma 3.1 of 39$]$ ) Let $\tilde{W}$ be a wedge orthogonal to $W$. Let $\rho_{\tilde{W}}$ and $\bar{\rho}_{\tilde{W}}$ denote the restrictions of $\rho$ and $\bar{\rho}$ to $\mathcal{A}(\tilde{W})$, then (id, $\rho_{\tilde{W}} \bar{\rho}_{\tilde{W}}$ ) is one dimensional and $V \in\left(\mathrm{id}, \rho_{\tilde{W}} \bar{\rho}_{\tilde{W}}\right) \cap \mathcal{A}(\tilde{\mathcal{O}})$, where $\tilde{\mathcal{O}}$ is any element of $\mathcal{L}$ G containing $\mathcal{O}$ and $\mathbf{j}_{W} \mathcal{O}$ with $\tilde{\mathcal{O}} \subset \tilde{W}$.

Proof. We remark that the existence of conjugates for finite statistics depends on Assumption IX and was discussed in the proof of 5.11. Since we are dealing with a sector, Assumption IX implies that (id, $\rho_{\tilde{W}} \bar{\rho}_{\tilde{W}}$ ) is one dimensional and contained in $\mathcal{A}(\tilde{\mathcal{O}})$. In fact, let $z$ yield $\rho$ in $\mathcal{O}$, i.e. $y(a)=\rho$ for $a=\mathcal{O}$, then the cocycle $\bar{z}$, defined by

$$
\bar{z}(b)=j\left(z\left(\mathbf{j}_{W} b\right)\right), \quad b \in \Sigma_{1},
$$

\footnotetext{
${ }^{9}$ Recall that $\tilde{\mathcal{O}}$ is in $\mathcal{L}$ if $\tilde{\mathcal{L}}$-duality holds either for $\tilde{\mathcal{O}}$ or for $\tilde{\mathcal{O}}^{\perp}$, cf. the discussion at beginning of Sect. 4.2 .
} 
yields $j \cdot \rho \cdot j$ in $\bar{a}=\mathbf{j}_{W} \mathcal{O}$. Let $\hat{b} \in \Sigma_{1}$ be defined by $\partial_{o} \hat{b}=a, \partial_{1} \hat{b}=\bar{a}$ and $|\hat{b}|=\tilde{\mathcal{O}}$. A simple computation shows that

$$
y(a)(\bar{z}(\hat{b})) V A=y(a) \bar{y}(a)(A) y(a)(\bar{z}(\hat{b})) V, \quad a \in \mathcal{A}(\tilde{W}) .
$$

Thus by Assumption IX, $y(a)(\bar{z}(\hat{b})) V \in \mathcal{A}(\mathcal{O})$. But $\bar{z}(\hat{b}) \in \mathcal{A}(|\hat{b}|)=\mathcal{A}(\tilde{\mathcal{O}})$. Hence $V \in \mathcal{A}(\tilde{\mathcal{O}})$ as claimed. Obviously, an isometry $V$ in $\left(\mathrm{id}, \rho_{\tilde{W}} \bar{\rho}_{\tilde{W}}\right)$ will implement $\rho_{W}$. Now a simple computation shows that $j(V) \in\left(\mathrm{id}, \bar{\rho}_{\mathbf{j}_{W} \tilde{W}} \rho_{\mathbf{j}_{W} \tilde{W}}\right)$. But $\mathbf{j}_{W} \tilde{W}=\tilde{W}$ since $W$ and $\tilde{W}$ are orthogonal. Hence, we may suppose that $V=j(V)$ and differs at most by a sign from the standard implementation of the restriction of $\rho$ to $\mathcal{A}(W)$.

Let $\pi$ be a representative of a sector with finite statistics and let $z$ be an associated cocycle. Let $\mathcal{O}$ be a diamond contained in the intersection of two wedges $W_{1}$ and $W_{2}$ and $\rho$ the object of $\operatorname{End} \mathcal{A}(\mathcal{O})$ associated with $z$. Write $j_{i}$ for the modular antilinear morphism associated with $\mathcal{A}\left(W_{i}\right)$ and $\bar{\rho}_{i}$ for $j_{i} \cdot \rho \cdot j_{i}, i=1,2$.

Lemma 5.13. Let $\rho, \bar{\rho}_{i}$ and $W_{i}, i=1,2$, be as above and suppose there exists a $g \in \tilde{G}$ with $W_{2}=g W_{1}$. The following identity between representations of the net $\mathcal{O}_{1} \mapsto \mathcal{A}\left(\mathcal{O}_{1}\right), \mathcal{O}_{1} \supset \mathcal{O}$, holds:

$$
\pi \bar{\rho}_{1}=\operatorname{Ad} U_{\pi}\left(\mathbf{j}_{1} g \mathbf{j}_{1} g^{-1}\right) \pi \bar{\rho}_{2} \operatorname{Ad} U\left(\mathbf{j}_{1} g \mathbf{j}_{1} g^{-1}\right)^{*},
$$

where $g \mapsto \mathbf{j}_{1} g \mathbf{j}_{1}$ denotes by abuse of notation the action of $\mathbf{j}_{1}$ lifted to $\tilde{G}$ and $\mathbf{j}_{1}:=\mathbf{j}_{W_{1}}$.

Proof. We have $J_{2}=U(g) J_{1} U(g)^{*}$, hence $J_{1} J_{2}=U\left(\mathbf{j}_{1} g \mathbf{j}_{1} g^{-1}\right)$ and $j_{1} j_{2}=\operatorname{Ad} U\left(\mathbf{j}_{1} g \mathbf{j}_{1} g^{-1}\right)$, therefore

$$
\bar{\rho}_{1}=\operatorname{Ad} U\left(\mathbf{j}_{1} g \mathbf{j}_{1} g^{-1}\right) \bar{\rho}_{2} \operatorname{Ad} U\left(\mathbf{j}_{1} g \mathbf{j}_{1} g^{-1}\right)^{*} .
$$

Thus by covariance

$$
\begin{gathered}
\rho \bar{\rho}_{1}=\rho \operatorname{Ad} U\left(\mathbf{j}_{1} g \mathbf{j}_{1} g^{-1}\right) \bar{\rho}_{2} \operatorname{Ad} U\left(\mathbf{j}_{1} g \mathbf{j}_{1} g^{-1}\right)^{*} \\
=\operatorname{Ad} U_{\pi}\left(\mathbf{j}_{1} g \mathbf{j}_{1} g^{-1}\right) \rho \bar{\rho}_{2} \operatorname{Ad} U\left(\mathbf{j}_{1} g \mathbf{j}_{1} g^{-1}\right)^{*}
\end{gathered}
$$

Lemma 5.14. Let $\rho, W_{1}$ and $W_{2}$ and $g$ be as in the previous lemma. Then there is a (unique) complex number $c\left(\rho, W_{1}, g\right)$ of modulus one such that

$$
U_{\pi}\left(\mathbf{j}_{1} g \mathbf{j}_{1} g^{-1}\right) V_{2} U\left(\mathbf{j}_{1} g \mathbf{j}_{1} g^{-1}\right)^{*}=c\left(\rho, W_{1}, g\right) V_{1}
$$

Proof. By Lemma 5.12, $V_{1} \in\left(\mathrm{id}, \rho_{\tilde{W}_{1}} \bar{\rho}_{\tilde{W}_{1}}\right)$. Furthermore, by the previous lemma, $U_{\pi}\left(\mathbf{j}_{1} g \mathbf{j}_{1} g^{-1}\right) V_{2} U\left(\mathbf{j}_{1} g \mathbf{j}_{1} g^{-1}\right)^{*}$ belongs to the same one dimensional space of intertwiners.

Lemma 5.15. Let $\rho$ and $\sigma$ be two endomorphisms associated with a given sector as above, the first localized in $W_{1} \cap W_{2}, W_{2}=g W_{1}$, the second in $h W_{1} \cap h W_{2}, g, h \in \tilde{G}$. Then $c\left(\rho, W_{1}, g\right)=c\left(\sigma, h W_{1}, h g h^{-1}\right)$. 
Proof. We first observe that if $\sigma=\operatorname{Ad} W^{*} \rho$ for some unitary $W \in \mathcal{A}\left(W_{1} \cap W_{2}\right)$, then $V_{i}^{\rho}=W^{*} J_{i} W^{*} J_{i} V_{i}^{\sigma}$ and this implies that $c\left(\sigma, W_{1}, g\right)=c\left(\rho, W_{1}, g\right)$. Then we note that $c\left(\rho, W_{1}, g\right)=c\left(\alpha_{h}^{-1} \rho \alpha_{h}, h W_{1}, h g h^{-1}\right)$, where $\alpha_{h}=\operatorname{Ad} U(h)$, because $U(h)$ establishes an isomorphism between the original structure and the structure transformed by $h$. Since $\alpha_{h}^{-1} \rho \alpha_{h}$ and $\sigma$ are associated with the same sector and both localized in $h W_{1} \cap h W_{2}$ and $h g h^{-1} h W_{1}=h W_{2}$, the result now follows.

The previous lemma shows that for the given sector there is a well defined function $c(W, g)$ satisfying

$$
c(W, g)=c\left(h W, h g h^{-1}\right)
$$

whenever $W \cap g W \neq \emptyset$.

Lemma 5.16. Let $W \in \mathcal{W}$. Then the function $g \mapsto c(W, g)$ is a local group character, namely for any $g, h \in \tilde{G}$ such that $W \cap g W \cap g h W \neq \emptyset$, we have

$$
c(W, g) c(W, h)=c(W, g h) .
$$

Proof. Choose associated endomorphisms localized in $W \cap g W \cap g h W$ and denote the involutions associated with $W$ and $g W$ by $\mathbf{j}_{1}$ and $\mathbf{j}_{2}$, respectively. Then from the definition of $c$ for the pairs $(W, g)$ and $(g W, h g W)$ and the equality

$$
\left(\mathbf{j}_{1} g \mathbf{j}_{1} g^{-1}\right)\left(\mathbf{j}_{2} h \mathbf{j}_{2} h^{-1}\right)=\mathbf{j}_{1} g \mathbf{j}_{1} g^{-1}\left(g \mathbf{j}_{1} g^{-1}\right) h\left(g \mathbf{j}_{1} g^{-1}\right) h^{-1}=\mathbf{j}_{1} h g \mathbf{j}_{1}(h g)^{-1}
$$

one obtains the relation

$$
c(W, g) c(g W, h)=c(W, g h)
$$

which means that the function $c$ is a local groupoid character. Then, making use of Lemma 5.15 we get

$$
c(W, g) c(W, h)=c\left(W, g h g^{-1}\right)=c\left(W,\left(g h g^{-1}\right) g\right)=c(W, g h) .
$$

In Proposition 5.10, we only used properties (b 1), (b 2). The rest of the argument makes essential use of further properties, more precisely, (b2) and (b3) are used in the following proposition, whilst (b3) and (b4), or rather, the orthogonality of Remark 5.1, are used to conclude the proof of Theorem 5.11.

Proposition 5.17. Under the given assumptions, we have $c\left(W, \exp \frac{\pi}{2} \mathfrak{h}\right)=1$.

Proof. Since $g \mapsto c(W, g)$ is a local representation, it is locally trivial on the commutator of $\widetilde{G}$, hence, by assumption (c), there exists $\varepsilon>0$ such that $c(W, \exp t \mathfrak{h})=1$ for $|t| \leq \varepsilon$. Because of assumption (b 4) the result follows by applying Lemma 5.16 sufficiently often.

Lemma 5.18. Let $\rho$ be an endomorphism associated with the sector and localized in $\mathcal{O} \subset W_{1} \cap W_{2}$, where $W_{1}$ and $W_{2}$ are orthogonal wedges, $W_{2}:=\exp \left(\frac{\pi}{2} \mathfrak{h}\right) W_{1}$ (cf. Remark 5.1). Let the standard implementations of its restriction to the algebras $\mathcal{A}\left(W_{1}\right), \mathcal{A}\left(W_{2}\right)$ be denoted by $V_{1}, V_{2}$ as before. Then the statistics parameter $\lambda_{\rho}$ can be written as $\lambda_{\rho}=V_{1}^{*} V_{2}^{*} V_{1} V_{2}$. 
Proof. As in 32], Lemma 3.5, we first show $\lambda_{\rho}=\rho\left(V_{1}^{*}\right) V_{1}$; indeed if $\rho^{\prime}$ is localized in $W_{1} \cap W_{2}^{\perp}$ and $u$ is a unitary in $\left(\rho, \rho^{\prime}\right)$ in $\operatorname{End} \mathcal{A}\left(W_{1}\right)$, then $u \in \mathcal{A}\left(W_{1}\right)$. Since $\left(W_{1} \cup W_{2}\right)^{\perp} \neq \emptyset, u^{*} A=u^{*} \rho^{\prime}(A)=\rho(A) u^{*}$, for $A \in \mathcal{A}\left(W_{2}\right)$. But $V_{1} \in \mathcal{A}\left(W_{2}\right)$ by Lemma 5.12. Thus $\rho\left(V_{1}^{*}\right) V_{1}=u^{*} V_{1}^{*} u V_{1}$. Now $\bar{\rho}_{1}:=j_{1} \cdot \rho \cdot j_{1}$ is localized in $W_{1}^{\perp} \cap W_{2}$ and, again since $\left(W_{1} \cup W_{2}\right)^{\perp} \neq \emptyset, \rho, \rho^{\prime}$ and $\bar{\rho}_{1}$ are comparable and $\hat{\rho}_{1}(u)=u$. Thus $V_{1}^{*} u V_{1}=\phi(u)$, where $\phi$ is the left inverse of $\rho$. Hence $\rho\left(V_{1}^{*}\right) V_{1}=u^{*} \phi(u)=$ $\phi(\varepsilon(\rho, \rho))=\lambda_{\rho}$. Now $V_{2} \in \mathcal{A}\left(W_{1}\right)$ and implements $\rho$ on $\mathcal{A}\left(W_{2}\right) . \quad \bar{\rho}_{2}:=j_{2} \cdot \rho \cdot j_{2}$ is localized in $W_{1} \cap W_{2}^{\perp}$ and since $\left(W_{1} \cup W_{2}\right)^{\perp} \neq \emptyset, \bar{\rho}_{2}\left(V_{1}\right)=V_{1}$ so we have

$$
V_{1}^{*} V_{2}^{*} V_{1} V_{2}=V_{1}^{*} \phi\left(V_{1}\right)=\phi\left(\rho\left(V_{1}^{*}\right) V_{1}\right)=\phi\left(\lambda_{\rho}\right)=\lambda_{\rho} .
$$

Before proceeding to the proof of Theorem 5.11, we prove a result about orthogonal wedges.

Lemma 5.19. Given two orthogonal wedges $W_{1}, W_{2}$ with reflections $\mathbf{j}_{1}$ and $\mathbf{j}_{2}$, there is a region $\mathcal{O}$ with non-empty causal complement which is invariant under $\mathbf{j}_{1}$ and $\mathbf{j}_{2}$.

Proof. Take $\mathcal{O}_{1}$ and $\mathcal{O}_{2}$ orthogonal to each other and contained in $W_{1} \cap W_{2}$, and set $\mathcal{O}=\mathcal{O}_{1} \cup \mathbf{j}_{1} \mathcal{O}_{1} \cup \mathbf{j}_{2} \mathcal{O}_{1} \cup \mathbf{j}_{1} \mathbf{j}_{2} \mathcal{O}_{1}$. Clearly $\mathcal{O}$ is causally disjoint from $\mathcal{O}_{2}$ and invariant under $\mathbf{j}_{1}$ and $\mathbf{j}_{2}$.

Proof of Theorem 5.11. We follow the reasoning of [32. Consider the two orthogonal wedges $W_{1}$, and $W_{2}=\exp \left(\frac{\pi}{2} \mathfrak{h}\right) W_{1}$ as in the preceding lemma and choose a representative endomorphism localized in a regular diamond $\mathcal{O} \in W_{1} \cap W_{2}$ and chosen such that there is an $\tilde{\mathcal{O}}$ containing $\mathcal{O}, \mathbf{j}_{1} \mathcal{O}, \mathbf{j}_{2} \mathcal{O}$ and $\mathbf{j}_{1} \mathbf{j}_{2} \mathcal{O}$. Then $\rho \bar{\rho}_{1} j_{2} \rho \bar{\rho}_{1} j_{2}=\rho \bar{\rho}_{2} j_{1} \rho \bar{\rho}_{2} j_{1}$ and are objects of $\operatorname{End} \mathcal{A}(\tilde{\mathcal{O}}) . \quad V_{1} J_{2} V_{1} J_{2}$ and $V_{2} J_{1} V_{2} J_{1}$ intertwine from the identity to this object in $\operatorname{End} \mathcal{A}(\tilde{\mathcal{O}})$. Thus $\beta_{\rho}:=\left(V_{1} J_{2} V_{1} J_{2}\right)^{*} V_{2} J_{1} V_{2} J_{1}$ is a scalar and we first show that it belongs to $(0,1]$, as in Lemma 3.4 in [32], observing that

$$
\beta_{\rho}=V_{1}^{*} U(\exp \pi \mathfrak{h}) V_{1}^{*} V_{2} U(\exp \pi \mathfrak{h}) V_{2}
$$

Then, by Equation 5.2 with $g=\exp \frac{\pi}{2} \mathfrak{h}$ and its adjoint and using the equation $c\left(W, \exp \frac{\pi}{2} \mathfrak{h}\right)=1$, proved in Proposition 5.17, we get

$$
V_{2}^{*} V_{1}=s_{\rho} U(\exp \pi \mathfrak{h}) V_{1}^{*} V_{2} U(\exp \pi \mathfrak{h})
$$

Inserting this equation into the expression for the statistics parameter given by Lemma 5.18 and comparing with Equation 5.4 we obtain

$$
\lambda_{\rho}=V_{1}^{*} V_{2}^{*} V_{1} V_{2}=s_{\rho} V_{1}^{*} U(\exp \pi \mathfrak{h}) V_{1}^{*} V_{2} U(\exp \pi \mathfrak{h}) V_{2}=s_{\rho} \beta_{\rho}
$$

and the result follows easily.

We conclude this subsection showing that the Spin and Statistics relation makes sense and is true for reducible covariant representations, too. Clearly the result follows from the irreducible case once we can show that the irreducible subrepresentations are still covariant. 
Proposition 5.20. Let $\pi$ be a representation satisfying the selection criterion and with finite statistics and covariant under the group $\widetilde{G}$. Then there exists a covariant representation $\left(\pi, U_{\pi}\right)$, where $U_{\pi}$ acts trivially on $\pi(\mathcal{A})^{\prime} . U_{\pi}$ is unique up to a one dimensional representation and any other choice of $U_{\pi}$ may be written as a product of $U_{\pi}$ and a representation $U_{\pi}^{o}$ contained in $\rho(\mathcal{A})^{\prime}$. In particular, each irreducible component of $\rho$ is $\widetilde{G}$-covariant.

Proof. Since $\pi$ has finite statistics, $\pi(\mathcal{A})^{\prime}$ and hence the centre of $\pi(\mathcal{A})$ are finite dimensional. Therefore if $\left(\pi, \widetilde{U}_{\pi}\right)$ is a covariant representation, $\widetilde{U}_{\pi}$ is trivial on such centre. Then, since $\widetilde{U}_{\pi}$ implements automorphisms of $\pi(\mathcal{A})$, it implements an action of $\widetilde{G}$ by automorphisms of $\rho(\mathcal{A})^{\prime}$, preserving any factorial component. Thus this action is implemented by a unitary representation $U^{o}$ in $\pi(\mathcal{A})^{\prime}$. Then $g \in \widetilde{G} \rightarrow \widetilde{U}_{r}(g) U^{o}(g)^{*}$ is a representation of $\widetilde{G}$ acting trivially on $\pi(\mathcal{A})^{\prime}$. Clearly such a representation decomposes into representations of the irreducible components of $\pi$, so these are $\widetilde{G}$-covariant.

Remark 5.21. The given proof of the spin and statistics relation does not rely on the continuity of the representations $U$ or $U_{\pi}$. Even Proposition 5.20 does not require continuity, because it relies on the fact that a connected Lie group acts trivially on a finite set and this is true without assuming continuity.

\section{Spin and Statistics under property $\left(c^{\prime}\right)$}

Now we give a proof of the Spin and Statisitics Theorem for rotationally symmetric spacetimes satisfying $\left(c^{\prime}\right)$ rather than $(c)$, such as the 3-dimensional SchwarzschildKruskal spacetime, for example.

Recall that in this case there is an involution $r_{W}:=\mathbf{j}_{W} \mathbf{j}_{W_{0}} \in G_{+}^{\uparrow}$ anticommuting with $\mathfrak{h}$ (cf. Remark 5.2 (vi)).

Let us denote by the subgroup of $G_{+}^{\uparrow}$ generated by $G_{0}$ and $r_{W}$ by $G_{1}$. If $r_{W}$ does not belong to $G_{0}, G_{1}$ is isomorphic to $G_{0} \times_{\sigma} \mathbb{Z}_{2}$, where $\sigma=\operatorname{Ad} r_{W}$. In the same way we can consider the group $\widetilde{G}_{1} \equiv \widetilde{G} \times_{\sigma} \mathbb{Z}_{2}$, where, by an abuse of notation, $\sigma$ lifted to $\widetilde{G}$ is still denoted by $\sigma$. We shall also denote the corresponding element in $\widetilde{G}_{1}$ by $r_{W}$. Clearly the covering map extends to an epimorphism from $\widetilde{G}_{1}$ to $G_{1}$. We want to show that any $\widetilde{G}$-covariant sector with finite statistics is $\widetilde{G}_{1}$-covariant, too.

Proposition 5.22. Let $\pi$ be an irreducible representation of $\mathcal{A}$ obeying the selection criterion, with finite statistics, and covariant under the group $\widetilde{G}$. Then it is covariant under $\widetilde{G}_{1}$.

Proof. Of course we may restrict to the case $r_{W} \notin G_{0}$. Since $r \equiv r_{W}$ is the product of $\mathbf{j}_{W}$ with $\mathbf{j}_{W_{0}}$, such reflections are implemented by the corresponding modular involutions $J, J_{0}$, and $j \rho j$ is equivalent to $j_{0} \rho j_{0}$, both being conjugate endomorphisms of $\rho$, there exists a unitary $U_{r}$ intertwining $\rho$ and $\alpha(r) \rho \alpha(r)$. Since $r^{2}=1, U_{r}^{2}$ implements the trivial action on $\mathcal{A}$, hence, $\rho$ being irreducibile, $U_{r}^{2}$ is a constant and we may choose $U_{r}$ selfadjoint. Then $U_{r} U_{\rho}(r g r) U_{r}$ is another representation of $\widetilde{G}$ realizing the covariance of $\rho$. Since $\rho$ is irreducible, we get $U_{r} U_{\rho}(g) U_{r}=\chi(g) U_{\rho}(r g r)$, where $\chi(g)$ 
is a character of $\widetilde{G}$. Applying this relation twice, we get $U_{\rho}(g)=\chi(g) \chi(r g r) U_{\rho}(g)$, namely $\chi(g) \chi(r g r)=1$. Now observe that, since $\chi$ is a Lie group representation, it is the exponential of a Lie algebra morphism $\kappa$ from the Lie algebra of $G_{0}$ to $\mathbb{R}$. Since $\widetilde{G}$ is simply connected, $\kappa / 2$ exponentiates to a character, which we denote by $\sqrt{\chi}$, whose square gives $\chi$, and we get

$$
U_{r} \sqrt{\chi}\left(g^{-1} U_{\rho}(g)\right) U_{r}=\sqrt{\chi}\left(r g^{-1} r\right) U_{\rho}(r g r),
$$

namely $\sqrt{\chi}\left(g^{-1}\right) U_{\rho}(g)$ and $U_{r}$ yield the required representation of $\widetilde{G}_{1}$.

Theorem 5.23. Let $\left(M, G_{+}, \mathbf{j}, \mathcal{W}\right)$ be a rotationally symmetric spacetime satisfying properties (a) and $(b)$ and $\left(c^{\prime}\right),(\mathcal{A}, U, \Omega)$ a covariant net verifying the mentioned axioms (I-III), (VI-IX), and let $\rho$ be a $\widetilde{G}$-covariant sector with finite statistics. Then the spin and statistics relation holds.

Proof. By property $\left(\mathrm{c}^{\prime}\right), s_{\rho}$ does not depend on $U_{\rho}$, as observed in Remark 5.2 (vi). Concerning the relation between spin and statistics, we may define a function $c(W, g)$, $g \in \widetilde{G}_{1}$, as in the proof of Theorem 5.11, which is indeed a local group representation namely, if $g, h \in \widetilde{G}_{1}$ verify $W \cap g W \cap g h W \neq \emptyset$, we have $c(W, g) c(W, h)=c(W, g h)$. Setting $\tilde{r}:=\exp \left(\frac{\pi}{2} \mathfrak{h}\right) r_{W} \exp \left(-\frac{\pi}{2} \mathfrak{h}\right)$, we get

$$
\tilde{r} W=\exp \left(\frac{\pi}{2} \mathfrak{h}\right) r_{W} \exp \left(-\frac{\pi}{2} \mathfrak{h}\right) W=\exp (\pi \mathfrak{h}) r_{W} W=W
$$

and $\tilde{r} \exp (t \mathfrak{h}) \tilde{r}=\exp (-t \mathfrak{h})$. Hence, for sufficiently small $t$,

$$
\begin{aligned}
c(W, \exp (2 t \mathfrak{h})) & =c(W, \tilde{r}) c(W, \tilde{r} \exp (t \mathfrak{h})) c(W, \exp (t \mathfrak{h})) \\
& =c(W, \tilde{r} \exp (t \mathfrak{h}) \tilde{r}) c(W, \exp (t \mathfrak{h})) \\
& =c(W, \exp (-t \mathfrak{h})) c(W, \exp (t \mathfrak{h})=1 .
\end{aligned}
$$

The proof now continues as in Theorem 5.11.

As before, the Spin and Statistics relation for reducible representations follows as soon as we prove that the irreducible representations are $\tilde{G}_{1}$-covariant, and this is a consequence of Proposition 5.20 and Proposition 5.22 .

Remark 5.24. Generally speaking, asking for an irreducible endomorphism to be covariant corresponds to asking for a projective representation of the group $G_{0}$, namely a representation of a central extension of $G_{0}$ by some subgroup of $\mathbb{T}^{1}$ implementing the action of $G_{0}$ on $\rho(\mathcal{A})$. This means that there may be an extension at the Lie algebra level, not only a covering. However, we are not aware of any physical example where non-trivial Lie algebra central extensions exist (for the Poincaré group on the two-dimensional Minkowski space, such non-trivial extensions exist, but are incompatible with the positive energy requirement). As a consequence, we have only treated the case of the universal covering. 


\subsection{Appendix. Equivalence between local and global inter- twiners in Minkowski spacetime}

In this appendix we prove that Assumption IX concerning the equivalence of local and global intertwiners holds for sectors localized in a wedge region of a Minkowski space of arbitrary dimension. The argument is a straightforward adaptation of that given in [32] for a conformal net on $S^{1}$.

In the following, $\mathcal{A}$ is a net of von Neumann algebras on the $d+1$-dimensional Minkowski spacetime. We assume Poincaré covariance with positive energy and uniqueness of the vacuum, additivity and Haag duality

$$
\mathcal{A}(\mathcal{O})=\mathcal{A}\left(\mathcal{O}^{\prime}\right)^{\prime}
$$

if $\mathcal{O}$ is either a double cone or a wedge region.

If $\rho, \sigma$ are endomorphisms of $\mathcal{A}$ localized in the wedge region $W$, we consider their intertwiner space $\left(\rho_{W}, \sigma_{W}\right):=\{T \in \mathcal{A}(W): \sigma(A) T=T \rho(A), \forall A \in \mathcal{A}(W)\}$. Ву duality we always have $(\rho, \sigma) \subset\left(\rho_{W}, \sigma_{W}\right)$.

Theorem A5.1 Let $W$ be a wedge region and $\rho, \sigma$ be endomorphisms with finite dimension localized in a double cone $\mathcal{O} \subset W$. Then

$$
\left(\rho_{W}, \sigma_{W}\right)=(\rho, \sigma) .
$$

Namely, if $T \in\left(\rho_{W}, \sigma_{W}\right)$ then $T$ intertwines the representations $\rho$ and $\sigma$ of $\mathcal{A}$.

In the following $\rho$ denotes an endomorphism with finite dimension of the quasilocal observable $\mathrm{C}^{*}$-algebra $\mathcal{A}$ localized in a double cone $\mathcal{O}$ contained in the wedge $W$. We may assume that $W=\left\{x \in \mathbb{R}^{d+1}:-x_{1}>\left|x_{0}\right|\right\}$.

We shall denote by $\mathbb{R}^{2}$ the 2 -dimensional $x_{0}-x_{1}$-plane and by $\mathbf{P}$ the corresponding 2-dimensional Poincaré group, namely the semidirect product of the 2-dimensional translations $\{T(x)\}_{x \in \mathbb{R}^{2}}$ and boosts $\{\Lambda(s)\}_{s \in \mathbb{R}}$ associated to $W$ : each $g \in \mathbf{P}$ can be written uniquely as a product $g=T(x) \Lambda(s)$.

The endomorphism $\rho$ restricts to an endomorphism of the $\mathrm{C}^{*}$-algebra associated with $W+x$ and then extends to the von Neumann algebra $\mathcal{A}(W+x)$, for $x_{1}>$ 0 , hence giving rise to an endomorphism the $C^{*}$-algebra $\mathcal{A}_{\infty}$, the norm closure of $\cup_{x \in \mathbb{R}^{2}} \mathcal{A}(W+x)$. We will still use $\rho$ to denote this endomorphism. Since $\mathbf{P}$ is simply connected, there is a unitary representation $U_{\rho}$ of $\mathbf{P}$ expressing the covariance of $\rho$ with respect to $\mathbf{P}$

$$
\beta_{g}(A)=U_{\rho}(g) A U_{\rho}(g)^{*}=z_{\rho}(g) U(g) A U(g)^{*} z_{\rho}(g)^{*}, \quad A \in \mathcal{A}_{\infty}, g \in \mathbf{P} .
$$

As the cocycle $z_{\rho}$ is a local operator by Haag duality (this is the essential point about the 2-dimensional $x_{0}-x_{1}$-net inherited from the higher dimensional original net) $\beta$ is an action of $\mathbf{P}$ by automorphisms of $\mathcal{A}_{\infty}$.

We consider now the semigroup $\mathbf{P}_{\mathbf{0}}$, the semidirect product of the boosts $\Lambda(s)$ with the positive translations, where we say that $T(x)$ is positive if $x \in \mathbb{R}^{2}$ with $x_{1}>\left|x_{0}\right|$. $\mathbf{P}_{\mathbf{0}}$ is an amenable semigroup and we need an invariant mean $m$ constructed as follows: first we average (with an invariant mean) over positive translations and then 
over boosts. Observe that $f \rightarrow \int_{\mathbf{P}_{\mathbf{0}}} f(g) d m(g)$ gives an invariant mean on all $\mathbf{P}$ vanishing on $f$ if, for any given $s \in \mathbb{R}$, the map $x \in \mathbb{R}^{2} \rightarrow f(T(x) \Lambda(s))$ vanishes on a right wedge.

Then we associate to $m$ the completely positive map $\Phi$ of $\mathcal{A}_{\infty}$ to $\mathcal{B}(\mathcal{H})$ given by

$$
\Phi(A):=\int_{\mathbf{P}_{\mathbf{0}}} z_{\rho}(g)^{*} A z_{\rho}(g) d m(g), \quad A \in \mathcal{A}_{\infty} .
$$

Lemma A5.2 $\Phi$ is a left inverse of $\rho$ on $\mathcal{A}_{\infty}$. Moreover $\Phi$ is locally normal, i.e. has normal restriction to $\mathcal{A}(W+x), x \in \mathbb{R}^{2}$, and $\mathbf{P}$-invariant, namely

$$
\Phi=\alpha_{g}^{-1} \Phi \beta_{g}, \quad g \in \mathbf{P} .
$$

We have set $\alpha_{g} \equiv \operatorname{Ad} U(g)$.

Proof. Let $A$ belong to $\mathcal{A}(W+x), x \in \mathbb{R}^{2}$. By formula 5.6

$$
\Phi(\rho(A))=\int_{\mathbf{P}_{\mathbf{0}}} \alpha_{g}\left(\rho\left(\alpha_{g^{-1}}(A)\right)\right) d m(g)=A
$$

because of the above property of $m$ since the integrand is constantly equal to $A$ on the set $g \in \mathbf{P}_{\mathbf{0}}: g^{-1} W \cap \mathcal{O}=\emptyset$. Then the localization of $\rho$ and Haag duality imply that the range of $\Phi$ is contained in $\mathcal{A}_{\infty}$.

Setting $E=\rho \cdot \Phi$ gives a conditional expectation of $\mathcal{A}_{\infty}$ onto the range of $\rho$ that restricts to a conditional expectation $E_{x}$ of $\mathcal{A}(W+x)$ onto $\rho(\mathcal{A}(W+x))$ if $W+x \supset \mathcal{O}$. Since $\rho_{W+x}$ is assumed to have finite index, $E_{x}$ is automatically normal [44]. Therefore $\Phi\left\lceil\mathcal{A}(W+x)=\rho_{W+x}^{-1} E_{x}\right.$ is normal for $x=\left(0, x_{1}\right)$ with $x_{1}>0$, hence for any $x$.

Concerning the $\mathbf{P}$-invariance of $\Phi$ we have, making use of the cocycle condition,

$$
\begin{aligned}
\alpha_{g}^{-1} \Phi \beta_{g}(A) & =\alpha_{g}^{-1}\left(\int_{\mathbf{P}_{\mathbf{0}}} z_{\rho}(h)^{*} \beta_{g}(A) z_{\rho}(h) d m(h)\right) \\
& =\alpha_{g}^{-1}\left(\int_{\mathbf{P}_{\mathbf{0}}} z_{\rho}(h)^{*} z_{\rho}(g) \alpha_{g}(A) z_{\rho}(g)^{*} z_{\rho}(h) d m(h)\right) \\
& =\int_{\mathbf{P}_{\mathbf{0}}} z_{\rho}\left(h g^{-1}\right)^{*} A z_{\rho}\left(h g^{-1}\right) d m(h)=\Phi(A)
\end{aligned}
$$

Corollary A5.3 $\varphi=\omega \Phi$ is a locally normal $\beta$-invariant state on $\mathcal{A}_{\infty}$, where $\omega=$ $(\cdot \Omega, \Omega)$.

Proof. We have $\varphi \beta_{g}=\omega \Phi \beta_{g}=\omega \alpha_{g} \Phi=\omega \Phi=\varphi$ and $\varphi$ is locally normal because both $\omega$ and $\Phi$ are locally normal. 
Let $\left\{\pi_{\varphi}, \xi_{\varphi}, \mathcal{H}_{\varphi}\right\}$ be the GNS triple associated with the above state $\varphi$ and $V$ be the unitary representation of $\mathbf{P}$ on $\mathcal{H}_{\varphi}$ given by $V_{g} A \xi_{\varphi}=\beta_{g}(A) \xi_{\varphi}$ for $A \in \mathcal{A}_{\infty}$. Notice that $V$ is strongly continuous because $\varphi$ is locally normal.

Lemma A5.4 If $\rho$ is irreducible then

$$
\varphi(x)=\int_{\mathbf{P}_{\mathbf{0}}} \beta_{g}(x) d m(g), \quad x \in \mathcal{A}_{\infty}
$$

Proof. If $A \in \mathcal{A}(W+x)$ and $B \in \mathcal{A}_{\infty}$ is localized in a double cone, the commutator function $\mathbb{R}^{2} \ni x \mapsto\left[\beta_{T(x) \Lambda(s)}(A), \rho(B)\right]=\beta_{T(x) \Lambda(s)}\left(\left[A, \rho\left(\alpha_{T(x) \Lambda(s)}^{-1}(B)\right]\right)\right.$ vanishes on a right wedge, hence $\left[\int_{\mathbf{P}_{\mathbf{0}}} \beta_{g}(A), \rho(B) d m(g)\right]=\int_{\mathbf{P}_{\mathbf{0}}}\left[\beta_{g}(A), \rho(B)\right] d m(g)=0$.

Since $\rho$ is locally normal, $\int_{\mathbf{P}_{\mathbf{0}}} \beta_{g}(A) d m(g)$ commutes with every $\rho(\mathcal{A}(W+x))$, thus with $\rho\left(\mathcal{A}_{\infty}\right)$; but $\rho$ being irreducible, it is therefore a scalar equal to its vacuum expectation value:

$$
\int_{\mathbf{P}_{\mathbf{0}}} \beta_{g}(A) d m(g)=\int_{\mathbf{P}_{\mathbf{0}}} \omega\left(\beta_{g}(A)\right) d m(g)=\int_{\mathbf{P}_{\mathbf{0}}} \omega\left(z_{g}^{*} A z_{g}\right) d m(g)=\omega \Phi(A)=\varphi(A),
$$

as $\omega$ is normal and $\alpha$-invariant.

Corollary A5.5 If $\rho$ is irreducible, the two-parameter unitary translation group $V(T(x))$ satisfies the spectrum condition.

Proof. One may repeat the proof of Corollary 2.7 of [32] for each of the one-parameter light-like unitary translation groups.

Corollary A5.6 If $\rho$ is irreducible, $\varphi$ is faithful on $\cup \rho(\mathcal{A}(W+x))$.

Proof. $\mathcal{A}_{\infty}$ is a simple $C^{*}$-algebra since it is the inductive limit of type III factors (that are simple $C^{*}$-algebras). Therefore $\pi_{\varphi}$ is one-to-one and the statement will follow if we show that $\xi_{\varphi}$ if cyclic for $\mathcal{B}_{x} \equiv \rho(\mathcal{A}(W+x))^{\prime}, x_{1}>0$. To this end we may use a classical Reeh-Schlieder argument. If $\psi \in \mathcal{H}$ is orthogonal to $\mathcal{B}_{x} \xi_{\varphi}$, and $x-y \in W$, then for all $A \in \mathcal{B}_{y}$ we have $\left(A \xi_{\varphi}, V(T(x)) \psi\right)=0$ for $x$ in a neighborhood of 0 , thus for all $x \in \mathbb{R}^{2}$ by the spectrum condition shown by Corollary A5.5. Hence, setting $\alpha_{x} \equiv \alpha_{T(x)}$ and $\beta_{x} \equiv \beta_{T(x)}, \psi$ is orthogonal to $\left(\cup_{x} \beta_{x}\left(\mathcal{B}_{y}\right)\right) \xi_{\varphi}$, thus $\psi=0$ because $\cup_{x} \beta_{x}\left(\mathcal{B}_{y}\right)$ is irreducible since

$$
\begin{aligned}
\left(\bigcup_{x} \beta_{x}\left(\mathcal{B}_{y}\right)\right)^{\prime} & =\bigcap_{x} \beta_{x}\left(\rho(\mathcal{A}(W+y))=\bigcap_{x} \rho\left(\alpha_{x}(\mathcal{A}(W+y))\right)\right. \\
& =\rho\left(\bigcap_{x} \alpha_{x}(\mathcal{A}(W+y))\right)=\bigcap_{x} \mathcal{A}(W+x)=\mathbb{C}
\end{aligned}
$$

by the local normality of $\rho$.

Proposition A5.7 $\left(\rho_{W+x}, \rho_{W+x}\right)$ does not depend on the wedge $W+x \supset \mathcal{O}$. 
Proof. We begin with the case where $\rho$ is irreducible and assume for convenience that $\overline{\mathcal{O}} \subset W$. Notice then that $\left(\rho_{W}, \rho_{W}\right)$ is finite-dimensional and, by covariance, globally $\beta_{g}$-invariant with $g$ in the subgroup of boosts because these transformations preserve $W$. Therefore $\left(\rho_{W}, \rho_{W}\right) \xi_{\varphi}$ is a finite-dimensional subspace of $\mathcal{H}_{\varphi}$ globally invariant for $V(\Lambda(s)), s \in \mathbb{R}$. By Proposition B.3 of [32] we thus have $V(T(x)) A \xi_{\varphi}=A \xi_{\varphi}$ for every element $A \in\left(\rho_{W}, \rho_{W}\right)$, thus $\beta_{T(x)}(A)=A$ because $\xi_{\varphi}$ is separating. It follows that if $A \in\left(\rho_{W}, \rho_{W}\right)$ and $B \in \mathcal{A}(W)$

$$
\left[A, \rho\left(\alpha_{g}(B)\right)\right]=\beta_{g}\left(\left[\beta_{g}^{-1}(A), \rho(B)\right]\right)=\beta_{g}([A, \rho(B)])=0
$$

namely

$$
A \in\left(\rho_{W}, \rho_{W}\right) \Rightarrow A \in(\rho, \rho)=\mathbb{C} .
$$

Since the converse implication is obvious by wedge duality we have the equality of the two intertwiner spaces.

Now if $\rho$ is any endomorphism with finite index, $(\rho, \rho)$ is finite-dimensional because $(\rho, \rho) \subset\left(\rho_{W}, \rho_{W}\right)$ and $\rho$ decomposes into a direct sum of irreducible endomorphisms of $\mathcal{A}_{\infty}$ which are covariant, therefore the preceding analysis shows that $\left(\rho_{W}, \rho_{W}\right)=(\rho, \rho)$ in this case, too. Since $(\rho, \rho)$ is translation invariant, we get $\left(\rho_{W+x}, \rho_{W+x}\right)=(\rho, \rho)$ whenever $\mathcal{O} \subset W+x$ and, since $x$ was arbitrary, the result follows.

Proof of Theorem A5.1. The case $\sigma=\rho$ follows immediately by Proposition A5.6: if $T \in\left(\rho_{W}, \rho_{W}\right)$ then $T$ also belongs to $\left(\rho_{\tilde{W}}, \rho_{\tilde{W}}\right)$ for any wedge $\tilde{W} \supset W$ hence by additivity $T$ is a self-intertwiner of $\rho$ on the whole algebra $\mathcal{A}$.

To handle the general case, consider a direct sum endomorphism $\eta:=\rho \oplus \sigma$ localized in $W$, then

$$
\operatorname{dim}\left(\eta_{W}, \eta_{W}\right)=\operatorname{dim}\left(\rho_{W}, \rho_{W}\right)+\operatorname{dim}\left(\sigma_{W}, \sigma_{W}\right)+2 \operatorname{dim}\left(\rho_{W}, \sigma_{W}\right)
$$

while

$$
\operatorname{dim}(\eta, \eta)=\operatorname{dim}(\rho, \rho)+\operatorname{dim}(\sigma, \sigma)+2 \operatorname{dim}(\rho, \sigma)
$$

therefore $\operatorname{dim}\left(\rho_{W}, \sigma_{W}\right)=\operatorname{dim}(\rho, \sigma)$ and since we always have $(\rho, \sigma) \subset\left(\rho_{W}, \sigma_{W}\right)$ these two intertwiner spaces coincide.

\section{Acknowledgments}

R.V. has been in part supported through the Operator Algebras Network funded by the EU under contract CHRX-CT94-0566. R.V. also wishes to thank all the members of the operator algebra group at the Dipartimento di Matematica, Università di Roma "Tor Vergata", for their kind hospitality in 1996.

Three of the authors (D.G., J.R., R.V.) would like to thank the Erwin Schrödinger Institute, Vienna, as well as the Organizers of the Workshop on Quantum Field Theory in September 1997, D. Buchholz and J. Yngvason, for the opportunity of participating the workshop. The excellent working conditions provided a basis for discussions relevant to the present paper.

We would also like to thank K.-H. Rehren for pointing out a gap in an earlier version of Section 4. 


\section{References}

[1] H. Araki, L. Zsido, "Extension of the structure theorem of Borchers and its application to half-sided modular inclusions" manuscript, preliminary version (1995), to appear

[2] H. Baumgärtel, M. Wollenberg, Causal nets of operator algebras, Akademie Verlag, Berlin, 1992

[3] J.K. Beem, P.E. Ehrlich, Global Lorentzian geometry, Marcel Dekker, New York, 1981

[4] J.J. Bisognano, E.H. Wichmann, "On the duality condition for quantum fields", J. Math. Phys. 17, 303 (1976)

[5] H.-J. Borchers, "The CPT-theorem in two-dimensional theories of local observables", Commun. Math. Phys. 143, 315 (1992)

[6] H.-J. Borchers, "On modular inclusion and spectrum condition", Lett. Math. Phys. 27, 311 (1993)

[7] H.-J. Borchers, "When does Lorentz invariance imply wedge duality?", Lett. Math. Phys. 35, 39 (1995)

[8] H.-J. Borchers, "Half-sided modular inclusions and the construction of the Poincaré Group", Commun. Math. Phys. 179, 703 (1996)

[9] H.-J. Borchers, D. Buchholz, "Global properties of vacuum states in de Sitter space", Ann. Inst. H. Poincaré 70, 23 (1999)

[10] J. Bros, H. Epstein, U. Moschella, "Analyticity properties and thermal effects for general quantum field theory on de Sitter space-time", Commun. Math. Phys. 196, 535 (1998)

[11] M. Brown, "Locally flat imbeddings of topological manifolds", Annals of Math. 75, $331(1962)$

[12] R. Brunetti, K. Fredenhagen, "Interacting quantum fields in curved space: Renormalizability of $\varphi^{4}$ ", in the Proceedings of the Conference "Operator algebras and quantum field theory" held in Rome, July 1996, S. Doplicher, R. Longo, J. Roberts, L. Zsido eds, International Press, 1997;

-, "Microlocal analysis and interacting quantum field theories: Renormalization on physical backgrounds", preprint math-ph/9903028

[13] R. Brunetti, K. Fredenhagen, M. Köhler, "The microlocal spectrum condition and Wick polynomials of free fields in curved spacetimes", Commun. Math. Phys. 180, 633 (1996)

[14] R. Brunetti, D. Guido, R. Longo, "Modular structure and duality in conformal Quantum Field Theory", Commun. Math. Phys. 156, 201 (1993) 
[15] R. Brunetti, D. Guido, R. Longo, "Group cohomology, modular theory and space-time symmetries", Rev. Math. Phys., 7, 57 (1994)

[16] R. Brunetti, D. Guido, R. Longo, "First quantization via BW property", in progress.

[17] D. Buchholz, S.J. Summers, "An algebraic characterization of vacuum states in Minkowski space", Commun. Math. Phys. 155, 442 (1993)

[18] D. Buchholz, O. Dreyer, M. Florig, S.J. Summers, "Geometric modular action and spacetime symmetry groups", preprint math-ph/9805026

[19] D. Buchholz, M. Florig, S.J. Summers, "Hawking-Unruh temperature and Einstein causality in anti-de Sitter space-time", hep-th/9905178

[20] C.J.S. Clarke, "A title of cosmic censorship", Class. Quantum Grav. 11, 1375 (1994)

[21] J. Dieckmann, "Cauchy surfaces in globally hyperbolic spacetimes", J. Math. Phys. 29, 578 (1988)

[22] J. Dimock, B.S. Kay, "Classical and quantum scattering theory for linear scalar fields on the Schwarzschild metric. I." Ann. Phys. (N.Y.) 175, 366 (1987)

[23] S. Doplicher, R. Haag, J.E. Roberts: "Fields, observables and gauge transformations I", Commun. Math. Phys. 13, 1 (1969)

[24] S. Doplicher, J.E. Roberts: "Endomorphisms of $C^{*}$-algebras, cross products and duality for compact groups", Ann. Math. 130, 75 (1989)

[25] S. Doplicher, J.E. Roberts: "Why there is a field algebra with a compact gauge group describing the superselection structure in particle physics", Commun. Math. Phys. 131, 51 (1990)

[26] W. Driessler, "On the structure of fields and algebras on null planes, I", Acta Phys. Austriaca 46, 63 (1977)

[27] K. Fredenhagen, R. Haag, "On the derivation of Hawking radiation associated with the formation of a black hole", Commun. Math. Phys. 127, 273 (1990)

[28] K. Fredenhagen, K.-H. Rehren, B. Schroer, "Superselection sectors with braid group statistics and exchange algebras". 1, Commun. Math. Phys. 125, 201 (1989); 2, Rev. Math. Phys. Special Issue, 111 (Dec. 1992)

[29] R. Geroch, "Domain of dependence", J. Math. Phys. 11, 437 (1970)

[30] D. Guido, R. Longo, "Relativistic invariance and charge conjugation in quantum field theory", Commun. Math. Phys. 148, 521 (1992)

[31] D. Guido, R. Longo, "An algebraic spin and statistics theorem", Commun. Math. Phys. 172, 517 (1995) 
[32] D. Guido, R. Longo, "The conformal spin and statistics theorem", Commun. Math. Phys. 181, 11 (1996)

[33] D. Guido, R. Longo, H.-W. Wiesbrock, "Extensions of conformal nets and superselection structures", Commun. Math. Phys. 192, 217 (1998)

[34] R. Haag, Local quantum physics, 2nd ed., Springer, Berlin, Heidelberg, New York, 1996

[35] S.W. Hawking, "Particle creation by black holes", Commun. Math. Phys. 43, $199(1975)$

[36] S.W. Hawking, G.F.R. Ellis, The large scale structure of space-time, Cambridge University Press, 1973

[37] B.S. Kay, "The double-wedge algebra for quantum fields on Schwarzschild and Minkowski spacetimes", Commun. Math. Phys. 100, 57 (1985)

[38] B.S. Kay, "Quantum fields in curved spacetime: Non global hyperbolicity and locality", in the Proceedings of the Conference "Operator algebras and quantum field theory" held in Rome, July 1996, S. Doplicher, R. Longo, J. Roberts, L. Zsido eds, International Press, 1997

[39] B.S. Kay, M.J. Radzikowski, R.M. Wald, "Quantum field theory on spacetimes with a compactly generated Cauchy-horizon", Commun. Math. Phys. 183, 533 (1997)

[40] B.S. Kay, R.M. Wald, "Theorems on the uniqueness and thermal properties of stationary, nonsingular, quasifree states on spacetimes with a bifurcate Killing horizon", Phys.Rep. 207, 49 (1991)

[41] M. Keyl, "Causal spaces, causal complements and their relations to quantum field theory", Rev. Math. Phys. 8, 229 (1996)

[42] B. Kuckert, "A new approach to spin and statistics", Lett. Math. Phys. 35, 319 (1995)

[43] H. Leutwyler, J.R. Klauder, L. Streit, "Quantum field theory on lightlike slabs", Nouvo Cimento 66A, 536 (1970)

[44] R. Longo, "Index of subfactors and statistics of quantum fields. I", Commun. Math. Phys. 126, 217 (1989)

[45] R. Longo, "On the spin-statistics relation for topological charges", in the Proceedings of the Conference "Operator algebras and quantum field theory" held in Rome, July 1996, S. Doplicher, R. Longo, J. Roberts, L. Zsido eds, International Press, 1997

[46] R. Longo, "An analogue of the Kac-Wakimoto formula and black hole conditional entropy", Commun. Math. Phys. 186, 451 (1997) 
[47] R. Longo, J.E. Roberts, "A theory of dimension", K-Theory 11, 103 (1997)

[48] M. Müger, "On soliton automorphisms in massive and conformal theories", Rev. Math. Phys. 11, 337 (1999)

[49] B. O’Neill, Semi-Riemannian geometry, Academic Press, New York, 1983

[50] M.J. Radzikowski, "Micro-local appraoch to the Hadamard condition in quantum field theory in curved space-time", Commun. Math. Phys. 179, 529 (1996)

[51] K.-H. Rehren, "Algebraic holography", preprint hep-th/9905179

[52] J.E. Roberts: Net cohomology and its applications to field theory. In: Quantum fields - algebras, processes, ed. L. Streit, pp. 239-268. Springer, Wien, New York, 1980

[53] J.E. Roberts: Lectures on algebraic quantum field theory. In: The algebraic theory of superselection sectors. Introduction and recent results, ed. D. Kastler, pp. 1-112. World Scientific, Singapore, New Jersey, London, Hong Kong 1990

[54] J.E. Roberts: "Some applications of dilation invariance to structural questions in the theory of local observables", Commun. Math. Phys. 37, 273 (1974)

[55] F. Rohrlich, "Null plane field theory", Acta Phys. Austriaca, Suppl. 8 (Conference Proc., Schladming 1971), 227 (1971)

[56] G.L. Sewell, "Quantum fields on manifolds: PCT and gravitationally induced thermal states", Ann. Phys. (N.Y.) 141, 201 (1982)

[57] S.J. Summers, R. Verch, "Modular inclusion, the Hawking temperature, and quantum field theory in curved spacetime", Lett. Math. Phys. 37, 145 (1996)

[58] M. Takesaki, Tomita's theory of modular Hilbert-algebras and its applications. Lecture Notes in Mathematics Vol. 128 Springer, Berlin-Heidelberg-New York 1970

[59] R. Verch, "Continuity of symplectically adjoint maps and the algebraic structure of Hadamard vacuum representations for quantum fields in curved spacetime", Rev. Math. Phys. 9, 635 (1997)

[60] R. Verch, "Notes on regular diamonds", preprint, available as ps-file at http://www.lqp.uni-goettingen.de/lqp/papers/

[61] R.M. Wald, General relativity, University of Chicago Press, 1984

[62] R.M. Wald, Quantum field theory in curved spacetime and black hole thermodynamics, University of Chicago Press, 1994

[63] R.M. Wald, "Gravitational collapse and cosmic censorship", gr-qc/9710068, to appear in "The Black Hole Trail", ed. by B. Iyer. 
[64] H.-W. Wiesbrock, "Half-sided modular inclusions of von Neumann algebras" Commun. Math. Phys. 157, 83 (1993)

[65] H.-W. Wiesbrock, "Conformal quantum field theory and half-sided modular inclusions of von Neumann algebras " Commun. Math. Phys. 158, 537 (1993)

[66] H.-W. Wiesbrock, "Symmetries and modular intersections of von Neumann algebras", Lett. Math. Phys. 39, 203 (1997)

[67] H.-W. Wiesbrock, "Modular intersections of von Neumann algebras is quantum field theory", Commun. Math. Phys. 193, 269 (1998)

[68] R. Zimmer, "Ergodic Theory of Semisimple Groups", Boston-Basel-Stuttgart: Birkhäuser, 1984 